\title{
Postmastectomy radiation therapy for patients with tissue expanders
}

by

Keren Mayorov

A thesis submitted to the Faculty of Graduate and Postdoctoral Affairs in partial fulfillment of the requirements for the degree of

Master of Science

in

\author{
Physics \\ Specialization in Medical Physics
}

Ottawa-Carleton Institute for Physics

Department of Physics

Carleton University

Ottawa, Ontario, Canada

November 2020

(C) 2020 Keren Mayorov 



\begin{abstract}
Temporary breast tissue expanders contain a metal port that can vary in position throughout the course of radiation treatments. In this study, the magnitude and the dosimetric effect of the inter-fractional positional variations of the metal port was quantified. The robustness of the three most common external beam treatment techniques (tangential 3DCRT, VMAT and helical Tomotherapy) was evaluated against the measured positional errors. Results showed that inter-fractional positional errors were generally small, but errors larger than $1.5 \mathrm{~cm}$ were also observed. The inter-fractional positional variations arise from a combination of internal port errors and patient registration errors. Patient registration errors were shown to have larger dosimetric effect on target coverage and nearby organs than internal port errors. VMAT and Tomotherapy treatments are more robust when patient registration errors are minimized. The misalignment of the metal port during patient registration is acceptable when a more optimal anatomy match can be achieved.
\end{abstract}




\section{Acknowledgements}

I wish to express my deepest gratitude to my supervisor, Dr. Elsayed Ali, who has been an invaluable mentor and teacher throughout this journey. His passion for knowledge sharing and ability to provide constructive guidance, have profoundly impacted my current work and will help shape my future endeavors.

I would like to extend my gratitude to Jamie Bahm and Pat Lacasse, therapists at The Ottawa Hospital Cancer Centre, for their clinical contribution to this work, and the many hours they have allocated for guiding and training me.

I wish to thank Dr. Byron Wilson, former resident at The Ottawa Hospital, for his continuous technical help during this study.

I am extremely grateful for the staff at The Ottawa hospital, faculty members at Carleton University and fellow graduate students who always supported me along the way.

This work would not have been possible without the generous financial support from The Queen Elizabeth II Graduate Scholarship in Sciences and Technology, the Department of Physics at Carleton University, Dr. Ali's NSERC Discovery Grant \#2017-06253 and the Medical Physics Department at The Ottawa Hospital.

Last but not least, I would like to acknowledge the unwavering support and encouragement of my partner, Dan, as well as my parents, Natalia and Andrey, my brother, Philipp and my grandparents. 


\section{Ethics and originality statement}

This thesis summarizes the most significant portion of the author's work during the course of her M.Sc. program at Carleton University.

This study is part of a Quality Improvement Initiative at the Ottawa Hospital Cancer Centre, and is exempt from a full ethics review. Patient data included in this study were anonymized, and the Ottawa Hospital confidentiality and ethics rules were followed. The Carleton Univeristy Research Ethics office deferred to the assessment made at The Ottawa Hospital (Mr. Gordon DuVal, Director).

Dr. Elsayed Ali supervised the project and provided input on all of its components, including the publications and the thesis. The clinical treatment plans were created by the therapists Jamie Bahm and Pat Lacasse, to ensure that clinical standards are met. The author of this thesis performed all the computational work, performed the delivery of all treatment plans, and performed all of the necessary data analysis. All figures and graphs presented in this document are the original production of the author. 


\section{Publications based on this thesis}

Peer-reviewed papers

1. K. Mayorov and E. Ali, Magnitude and dosimetric impact of inter-fractional positional variations of the metal port of tissue expanders in postmastectomy patients treated with radiation, Phys. Imaging Radiat. Oncol. 16, 37-42 (2020).

2. K. Mayorov, P. Lacasse and E. Ali, Robustness of three external beam treatment techniques against inter-fractional positional variations of the metal port in breast tissue expanders, submitted to Phys. Imaging Radiat. Oncol. November 2020.

\section{Conference Abstracts}

1. K. Mayorov and E. S. M. Ali, "Dosimetric impact of interfractional positional variation of the metal port of tissue expanders in postmastectomy patients treated in Tomotherapy", $65^{\text {th }}$ COMP Annual Scientific Meeting, Kelowna, BC, Canada, Sept. 2019, Finalist at the Young Investigator's Symposium. Published in Med. Phys. 46: 5373-5374.

2. K. Mayorov and E. S. M. Ali, "Robustness of three treatment modalities against inter-fractional positional variations of the metal port in breast tissue expanders", Virtual Joint AAPM/COMP Scientific Meeting, Jul. 2020, Therapy Blue Ribbon ePoster. 


\section{Table of contents}

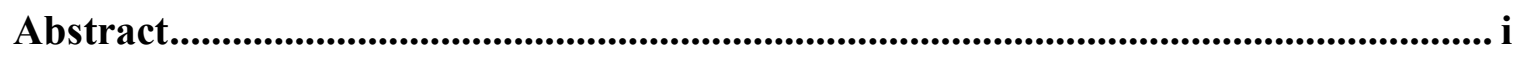

Acknowledgements .....................................................................................................

Ethics and originality statement .....................................................................................iii

Publications based on this thesis........................................................................... iv

Table of contents .......................................................................................................................... v

Acronyms ................................................................................................................................. vii

List of figures....................................................................................................................................... ix

Chapter 1 : Introduction ............................................................................................................... 1

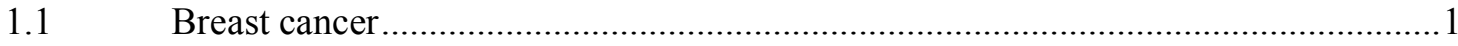

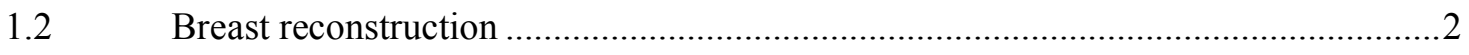

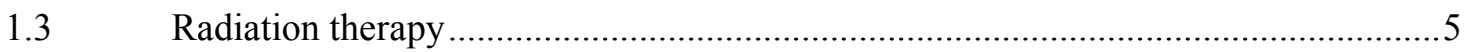

$1.4 \quad$ Radiation therapy techniques for breast cancer ...................................................11

1.4.1 Three-Dimensional Conformal Radiation Therapy (3DCRT) ………………….......12

1.4.2 Volumetric Modulated Arc Therapy (VMAT) ………………………………….......13

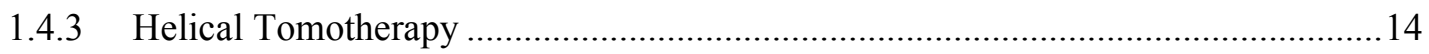

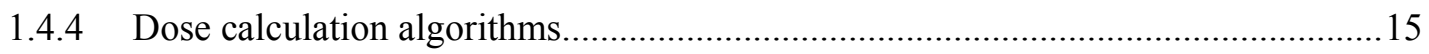

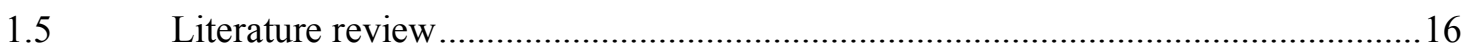

1.6 Intra-fraction and inter-fraction motion.............................................................2

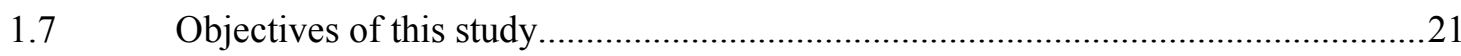

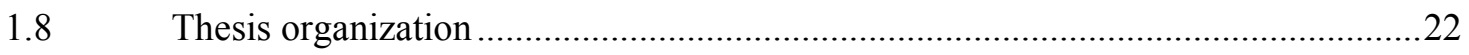

Chapter 2 : Measuring inter-fractional positional errors ................................................ 23

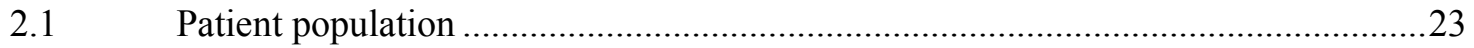

2.2 Patient registration and measuring positional errors..............................................24

2.3 Results: measured inter-fractional positional errors .............................................2 
Chapter 3 : Robustness against internal port positional errors .................................. 31

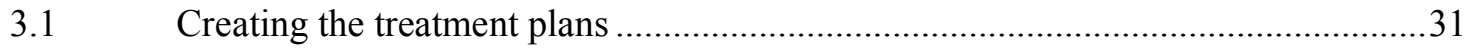

3.2 Modelling internal port positional errors ...........................................................

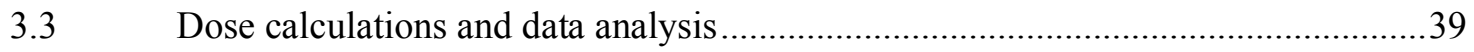

3.4 Results: dosimetric effect of daily measured errors and a systematic error .............41

Evaluating density override as a planning strategy ..........................................52

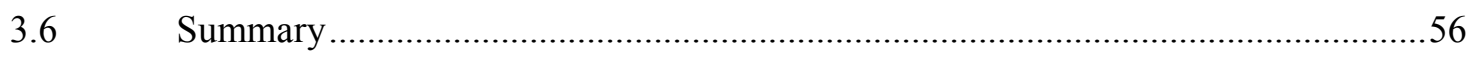

Chapter 4 : Robustness against patient registration errors....................................57

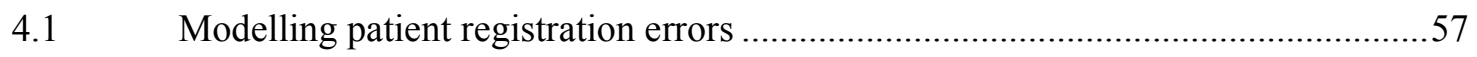

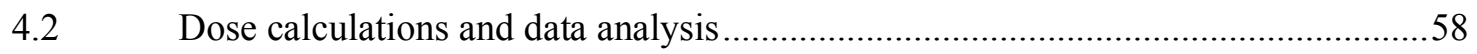

4.3 Results: dosimetric effect of daily measured errors and a systematic error ............60

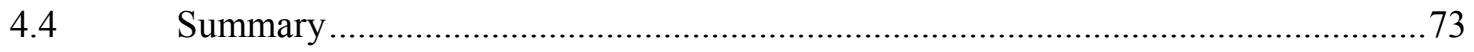

Chapter 5 : Special case............................................................................................................ 74

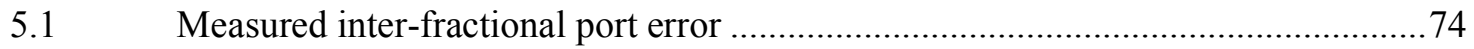

5.2 Results: dosimetric effect of daily measured errors............................................ 77

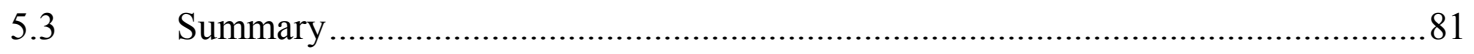

Chapter 6 : Overall summary, conclusions and future work .................................. 82

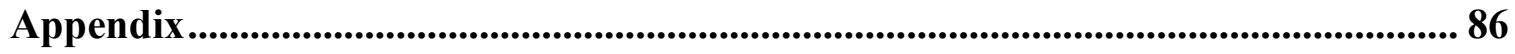

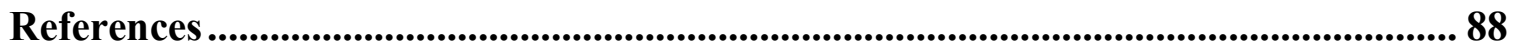




\section{Acronyms}

3D Three-Dimensional

3DCRT Three-Dimensional Conformal Radiation Therapy

BIA-ALCL Breast Implant-Associated Anaplastic Large Cell Lymphoma

CC Collapsed Cone (a dose calculation algorithm)

CS Convolution/Superposition (a dose calculation algorithm)

CT Computed Tomography

CTV Clinical Target Volume

DICOM Digital Imaging and Communications in Medicine

DQA Data Quality Assurance

DVH Dose-Volume Histogram

GTV Gross Tumor Volume

Gy Gray

HU Hounsfield Unit

ICRU International Commission on Radiation Units and Measurements

IMRT Intensity Modulated Radiation Therapy

IV Irradiated Volume

IVDT Image Value-to-Density Table

kVCT kilovoltage Computed Tomography

LINAC Linear accelerator

MC Monte Carlo (a dose calculation algorithm)

MLC Multi-Leaf Collimator

MRI Magnetic Resonance Imaging 


$\begin{array}{ll}\text { MVCT } & \text { Megavoltage Computed Tomography } \\ \text { OAR } & \text { Organ At Risk } \\ \text { PDD } & \text { Percent Depth Dose } \\ \text { PET } & \text { Positron Emission Tomography } \\ \text { PTV } & \text { Planning Target Volume } \\ \text { RED } & \text { Relative Electron Density } \\ \text { ROI } & \text { Region Of Interest } \\ \text { Rx } & \text { Prescription dose } \\ \text { TLD } & \text { Thermoluminescent Dosimeter } \\ \text { TOHCC } & \text { The Ottawa Hospital Cancer Centre } \\ \text { TPCT } & \text { Treatment Planning Computed Tomography } \\ \text { TPS } & \text { Treatment Planning System } \\ \text { TR } & \text { Treated Volume } \\ \text { V } x \text { Gy } & \text { Fraction of volume receiving } x \text { Gy } \\ \text { VMA } & \text { Folumetric Modulated Arc Therapy } \\ \text { Re } & \end{array}$




\section{List of figures}

Figure 1.1: Schematic diagram of postmastectomy breast reconstruction ....................... 3

Figure 1.2: (a) Deflated tissue expander (b) metal port ............................................. 3

Figure 1.3: Schematic diagram of the metal port..................................................... 4

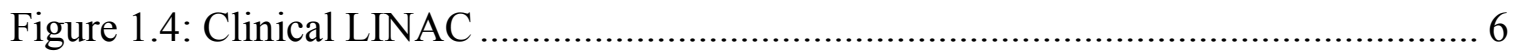

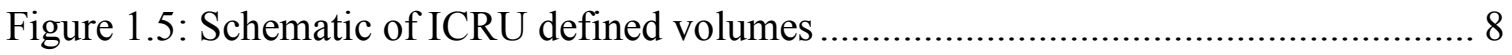

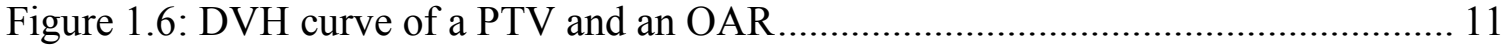

Figure 1.7: Schematic beam configurations of different breast irradiation techniques .... 13

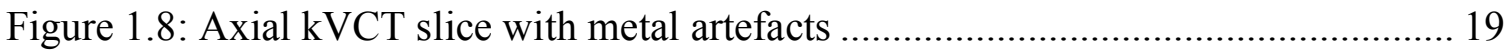

Figure 2.1: Axial TPCT slice showing chest wall curvature of two patients .................. 24

Figure 2.2: Checkered view of MVCT superimposed on TPCT ….............................. 26

Figure 2.3: Distribution of port positional errors for all patients................................ 27

Figure 2.4: Boxplot of port positional errors per patient ....................................... 29

Figure 3.1: HU thresholding when metal artefacts are present ..................................... 36

Figure 3.2: Identifying the voxels that belong to the metal port.................................. 37

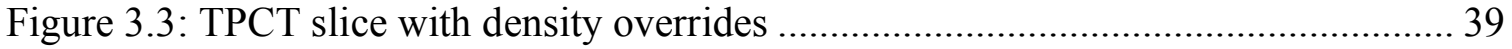

Figure 3.4: Originally planned dose distributions................................................... 42

Figure 3.5: Percent dose difference maps for daily measured internal port errors .......... 43

Figure 3.6: Percent dose difference map for a systematic port positioning error ............. 44

Figure 3.7: Boxplot of percent point dose differences inside the ROI ......................... 45

Figure 3.8: DVH of the ROI for a represetative patient ........................................... 47

Figure 3.9: Boxplot of the change in $\mathrm{V}_{100 \% \mathrm{Rx}}$ in the ROI........................................... 48

Figure 3.10: DVH of the ipsilateral lung and heart for a representative patient ..............50 


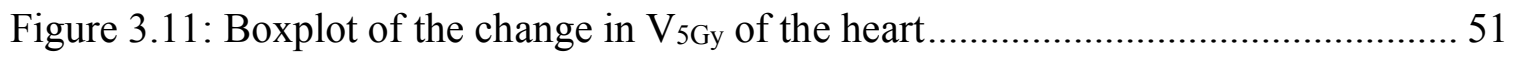

Figure 3.12: Boxplot of the change in $\mathrm{V}_{20 \mathrm{~Gy}}$ of the ipsilateral lung............................... 51

Figure 3.13: Change in $\mathrm{V}_{100 \% \mathrm{Rx}}$ of the ROI when the metal port is overridden ............... 54

Figure 4.1: Percent dose difference maps for daily variable patient registration errors ... 62

Figure 4.2: Percent dose difference maps for a systematic patient registration error ....... 63

Figure 4.3: Boxplot of percent point dose differences in the ROI ................................ 64

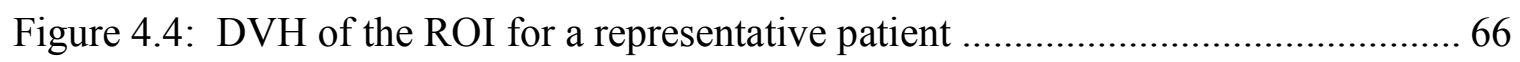

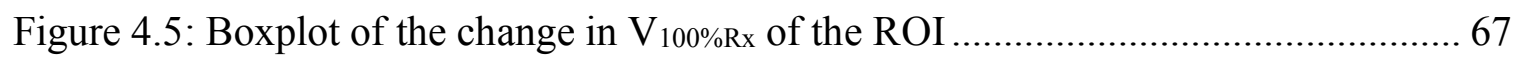

Figure 4.6: DVH of the ipsilateral lung and heart for a representative patient................ 69

Figure 4.7: Boxplot of the change in $\mathrm{V}_{20 \mathrm{~Gy}}$ of the ipsilateral lung................................. 70

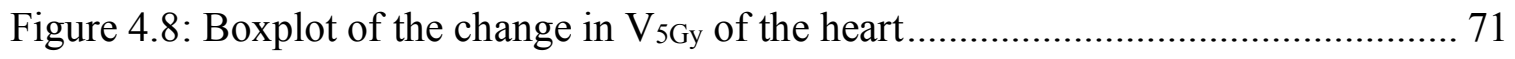

Figure 5.1: Checkered view of an MVCT superimposed on the TPCT ......................... 75

Figure 5.2: Distribution of port positional error for a special case ............................. 76

Figure 5.3: Percent dose difference maps for daily variable internal port errors ............ 78

Figure 5.4: DVH of the ROI for a special case ...................................................... 80 


\section{Chapter 1: Introduction}

\subsection{Breast cancer}

Breast cancer is the most commonly diagnosed cancer among Canadian women, with 27,400 new cases and 5,100 deaths projected in 2020. ${ }^{1}$ The incidence rate in Canadian females increased between 1984 and 1991 by $2.1 \%$, mainly attributing to increased availability of mammography screening. ${ }^{2}$ In addition, the breast cancer mortality rate has been declining since the 1980s, with a downward trend of $-2.3 \%$ per year between 1994 and 2015. This is most likely due to increased rates of early diagnosis (increased availability of mammography), and the use of more effective therapies. ${ }^{2}$

Current approaches for managing breast cancer include surgery, radiation therapy, systemic therapy and a combination of multiple therapies. There are two main types of surgery for breast cancer, lumpectomy and mastectomy, and the selected approach depends on several factors, such as the stage and location of the disease. ${ }^{3}$ In breast-conserving surgery, or lumpectomy, the tumor with a margin of normal tissue is removed, conserving the overall shape of the breast. In a mastectomy, all the breast tissue is removed. It has been shown that the long-term survival of women who undergo breast-conserving surgery is the same for women who undergo mastectomy. ${ }^{4}$ Nevertheless, one study showed that $30 \%$ of breast cancer patients still receive a mastectomy as the primary treatment. Also, patient involvement in the decision process was associated with greater mastectomy rates. There has been a recent trend of women undergoing contralateral prophylactic mastectomy (risk reducing mastectomy of the healthy breast) instead of breast conserving surgery. ${ }^{5}$ 
Advances in plastic surgical techniques have made breast reconstruction a favorable option post mastectomy, and partially explains the increasing trend of bilateral mastectomies. ${ }^{6}$

\subsection{Breast reconstruction}

The percentage of women who choose to undergo breast reconstruction after mastectomy has increased in the past decade, ${ }^{7}$ with implant-based reconstruction dominating over autologous reconstruction. ${ }^{8}$ Modern techniques of postmastectomy breast reconstruction include the use of autologous tissue, prosthetics and the combination of the two. In an autologous reconstruction, tissue (skin, fat, muscle) from a different part of the body is used to rebuild the shape of the breast after a mastectomy. The most common form of a prosthetic reconstruction involves tissue expansion, a technique pioneered by Radovan. ${ }^{9}$ An inflatable silicone balloon (i.e., temporary tissue expander) is implanted at the site of the mastectomy which is subsequently expanded though a series of saline injections with 7-days intervals. This is done to recover the lost tissue in the mastectomy, enabling a natural appearing breast mound. Once the expansion is complete, the temporary tissue expander is replaced with a permanent implant in a second surgery. The expander-based reconstruction is illustrated in the schematic diagram in Figure 1.1. 

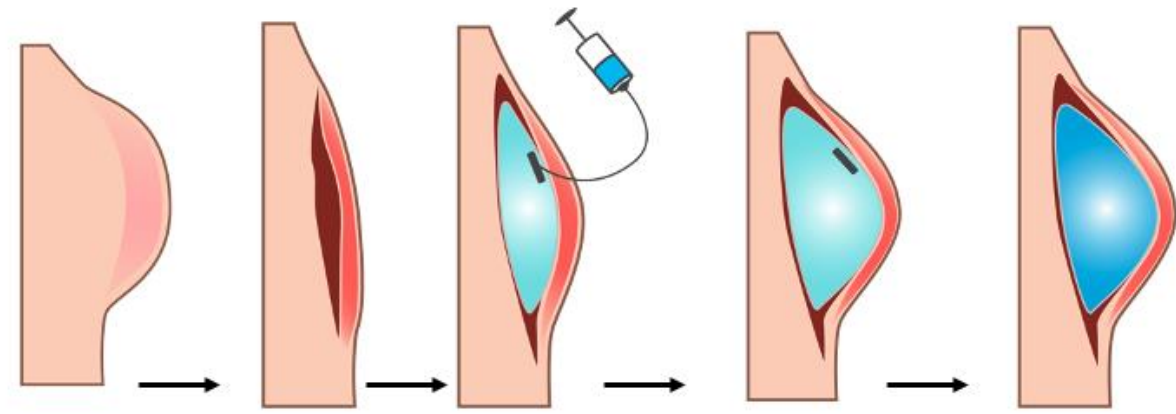

Figure 1.1: Schematic diagram of postmastectomy expander-implant reconstruction. A temporary tissue expander is inserted at the site of the mastectomy to stretch the overlaying skin with gradual saline injections. The temporary tissue expander is then replaced with a permanent implant.

The temporary tissue expander contains a metal port that facilitates the location of the injection site. The port is made of a rare-earth permanent magnet which is located with an external device. Once the metal port is located, a needle is inserted through the injection site until the needle is stopped by the needle guard. The expander is then filled with the necessary amount of sterile saline solution. A commonly used tissue expander is the Natrelle ${ }^{\circledR} 133$ (Allergan, Santa Barbara, CA) shown in Figure 1.2 along with its MAGNA$\mathrm{SITE}^{\circledR}$ metal port.
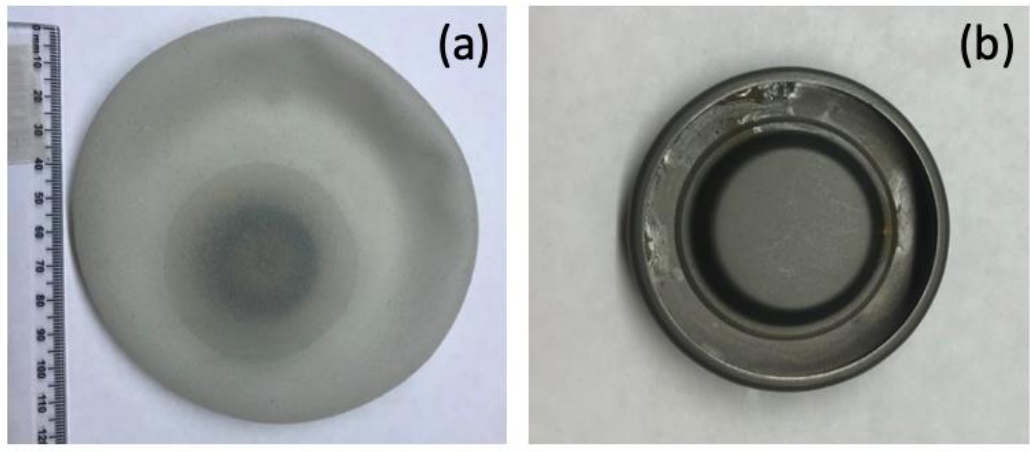

Figure 1.2: (a) Photograph of a commonly used tissue expander when deflated with the metal port in place.

(b) Close-up view of a metal port of the temporary tissue expander that contains a magnetic core encapsulated in a titanium shell. 
The dimensions of the metal port were previously measured in the clinic. The magnetic disc in the center consists of Samarium with a nominal density of $7.0 \mathrm{~g} / \mathrm{cm}^{3}$, encapsulated in a titanium shell with a nominal density of $4.7 \mathrm{~g} / \mathrm{cm}^{3}$. The physical dimensions of the metal port and its components are shown in Figure 1.3.

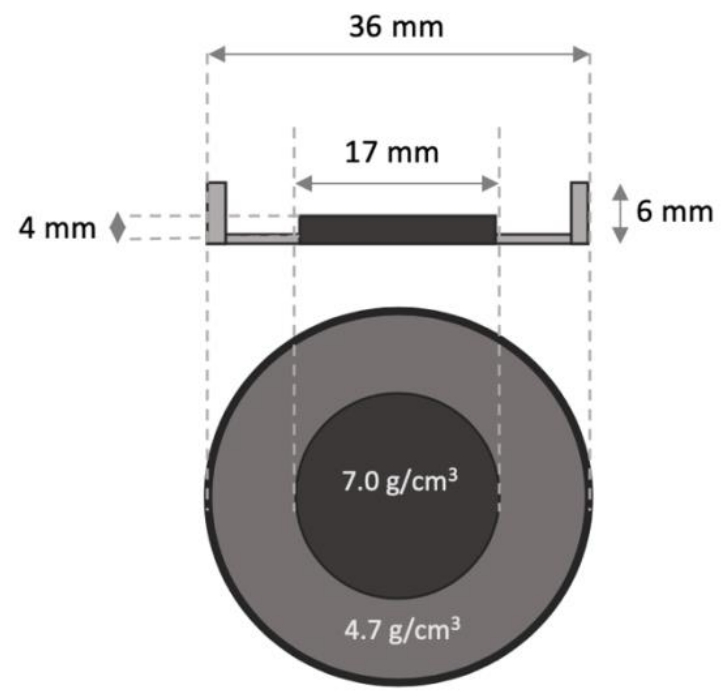

Figure 1.3: Schematic diagram of the metal port from a side and a top view along with its dimensions and nominal densities. This is the most commonly used metal port in tissue expanders, and all postmastectomy patients with tissue expanders included in this study have this metal port.

Breast reconstruction can take place at the time of mastectomy during the same anesthetic. This is also known as immediate breast reconstruction. Alternatively, a patient can undergo a delayed reconstruction months, or even years later. This would require an additional surgical procedure to insert the tissue expander, and longer hospitalization time. When compared to mastectomy only, immediate breast reconstruction was shown to be oncologically safe and not have an effect on recurrence rates and overall survival. ${ }^{10}$ Immediate reconstruction offers superior aesthetic results over delayed reconstruction. ${ }^{11}$ In addition, immediate reconstruction improves the psychological morbidity and self-esteem 
of patients, and was shown to improve health-related quality of life over the first postoperative years. ${ }^{12}$

\subsection{Radiation therapy}

Radiation therapy is the use of ionizing radiation to target and destroy cancer cells, with the goal to cure the disease or for palliative care. When combined with other treatment modalities such as surgery, it can be administered before the surgery (neoadjuvant therapy) with the aim of shrinking tumor, or, it can be administered after the surgery (adjuvant therapy) to ensure all microscopic disease is destroyed. ${ }^{13}$ In an external beam radiation therapy, a beam of ionizing radiation (e.g., photons and electrons) is delivered from outside the patient and is targeted to the location of the tumor.

External beam radiation therapy with megavoltage photon beams is the most common form of radiation treatment. The vast majority of external beam radiation therapy is delivered by a linear accelerator (LINAC). A LINAC is a device that uses high energy electromagnetic waves to accelerate electrons through a linear tube. ${ }^{14}$ The high-energy electrons can be used to generate x-rays. In the photon operating mode of the LINAC, bremsstrahlung x-rays are produced when the electrons are incident on a high atomic number target, such as tungsten. This produces $\mathrm{x}$-rays with a spectrum of energies, with a maximum energy equal to the maximum energy of the near-monoenergetic incident electron beam. The photon operating mode of LINACs is therefore specified in terms of the accelerating potential (e.g., $6 \mathrm{MV}$ photons). Figure 1.4 shows a photograph of an Elekta LINAC unit from The Ottawa Hospital Cancer Centre (TOHCC), taken with permission by the author. 


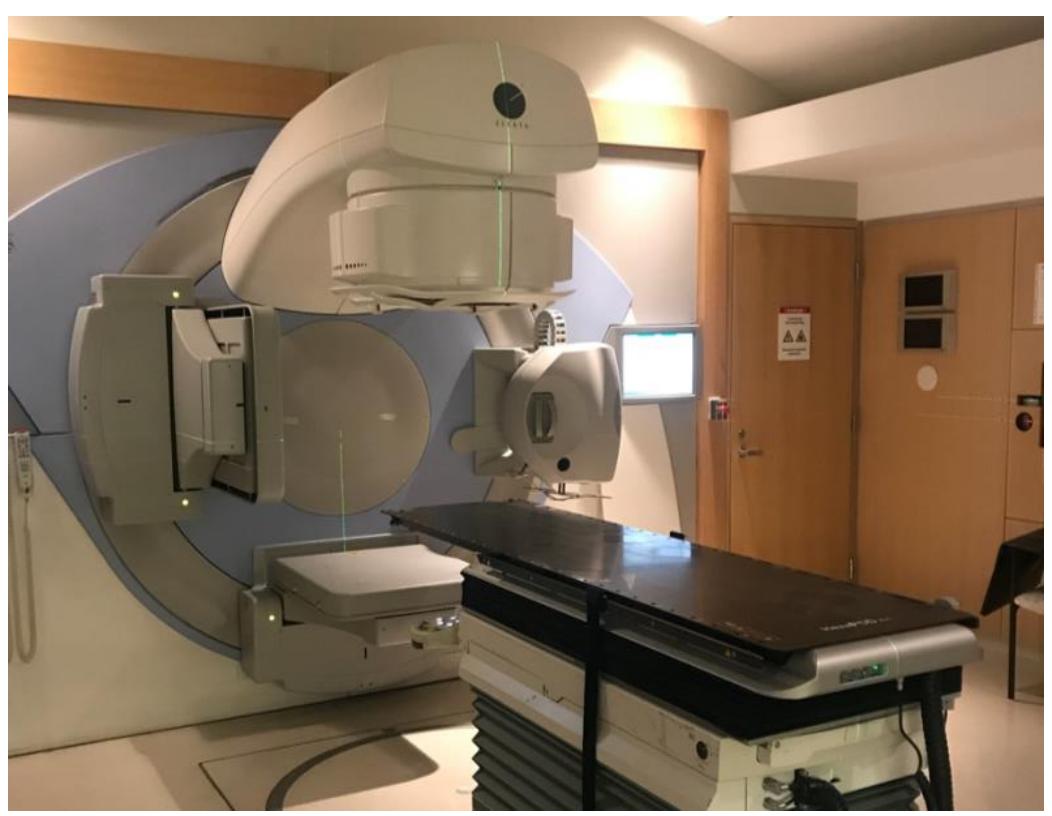

Figure 1.4: The Elekta synergy LINAC unit at TOHCC. The computational model of this LINAC was used to generate and calculate clinical plans for this study.

In an external beam radiation therapy, the first step in the treatment planning process is to determine the location of the tumor and its extent, and the location of critical nearby structures. This is achieved with the modern available imaging modalities such as Computed Tomography (CT), Magnetic Resonance Imaging (MRI), Positron Emission Tomography (PET), etc., and at times a combination of a few imaging modalities is used, depending on the type of cancer and the physiological attributes of the tumor. ${ }^{15} \mathrm{CT}$ is the main imaging modality used in radiation oncology, and the simulation scan is referred to in this thesis as the Treatment Planning CT (TPCT). In CT imaging, the linear attenuation coefficient, $\mu$, is the quantity that is being measured by passing an x-ray fan-beam through the patient. The projections are then reconstructed and stacked to create a threedimensional (3D) image. A typical CT slice consists of 512x512 pixels representing the 
CT numbers that are expressed in Hounsfield Units (HU). The CT number relates to the attenuation coefficient by

$$
\text { CT number }(\text { in } H U)=\frac{\mu-\mu_{w}}{\mu_{w}} \cdot 1000
$$

where $\mu_{w}$ is the attenuation coefficient of water. ${ }^{16}$ For radiation oncology purposes, the CT scans are acquired on CT-simulators, which have similar image quality to diagnostic scanners. CT-simulators differ from diagnostic scanners in that they have lasers for reference setup, flat couchtop, bigger bore for accessories, and a software dedicated to oncology requirements. The CT simulation scan is done in order to replicate the intended treatment position of the patient, because this $\mathrm{CT}$ is then used for treatment planning, where the dose delivered to the patient is calculated.

The next step in the treatment planning process is image segmentation. The goal of image segmentation is to locate anatomical structures relevant to the clinical objective of the treatment and contour their boundaries on the TPCT. ${ }^{17}$ Currently in the clinic both manual and automatic segmentation tools are utilized. ${ }^{18}$ Thresholding methods are used to contour interfaces with sharp differences in HU values, such as the lungs. ${ }^{19}$ In manual segmentation, anatomical structures are contoured manually on each transaxial slice of the CT to create a closed contour around relevant structures.

The International Commission on Radiation Units and Measurements (ICRU) established a systematic approach for defining the volumes related to the tumor and normal tissue. ${ }^{20}$ The Gross Tumor Volume (GTV) is defined as the gross demonstratable extent of the tumor detected in the diagnostic modality. In postmastectomy irradiation, where the gross tumor is resected and there is no GTV to define, only the Clinical Target Volume is used. The Clinical Target Volume (CTV) is defined as the volume containing the GTV 
with a margin to include subclinical malignant disease. The Planning Target Volume (PTV) is the volume including the CTV with a margin that accounts for internal (size, shape and position of (TV) and setup uncertainties. The PTV is the geometrical volume the dose is prescribed to ensure that an adequate dose is delivered to the CTV. The Treated Volume (TR) is the volume of tissue receiving at least the dose required to achieve the goal of the treatment, and the Irradiated Volume (IV) is the volume of tissue receiving more than an acceptable normal tissue threshold. A schematic representation of the above-mentioned volumes is shown in Figure 1.5.

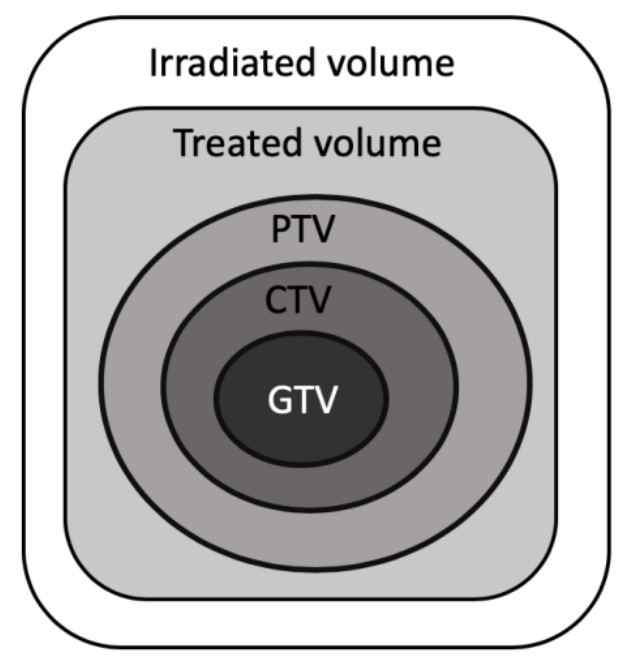

Figure 1.5: Schematic representation of the tumor related volumes defined by the ICRU. The GTV is expanded to the CTV to include subclinical disease, and the PTV is an expansion of the CTV to include internal and setup uncertainties.

An Organ At Risk (OAR) is defined as a structure that some irradiation of the structure can cause significant damage or morbidity to the patient. Normal structures are divided into "serial", "parallel" and "serial-parallel" organs. Serial organs are made up of a chain of functional units, where all units need to be intact to be functional. An example 
of a serial organ in the spinal cord. A parallel organ is made up of functional units that are independent from each other, where the damage to one functional group does not affect the other functional groups. For instance, the lung is a parallel organ. Serial-parallel organs are characterized by both organizations, such as the kidneys.

The radiation oncologist is responsible for delineating the target, and specifying clinical objectives, such as the prescription dose $(\mathrm{Rx})$ for the target. The $\mathrm{Rx}$ has units of energy per unit mass $(\mathrm{J} / \mathrm{kg})$, or gray $(\mathrm{Gy})$. Clinical treatment planning is done on a commercially available Treatment Planning System (TPS). In the TPS, various beam models and dose calculation algorithms (e.g., Monte Carlo and Collapsed Cone) are used to calculate dose distributions on the $3 \mathrm{D}$ patient data obtained from the simulation scan. The main goal of treatment planning is to find an arrangement of beams and their corresponding intensities that produce a dose distribution around the PTV that meets the clinical endpoint, while minimizing the dose to surrounding healthy tissue and OARs. There are two techniques that are used for treatment planning: forward planning and inverse planning. Forward planning is the conventional planning approach where the treatment planner applies and modifies the beams while assessing their effect on the dose distribution. In inverse planning, on the other hand, the optimization process is done computationally, given some pre-defined objectives and constraints. The application of these techniques and algorithms in the treatment of breast cancer will be discussed in more detail in the next subsection.

After the simulation scan and treatment planning, there is the step of accurately delivering the planned treatment. With current radiation techniques delivering highly conformal fields, it is critical that the patient setup on the treatment couch exactly matches 
the setup in the simulation scan. Various immobilization devices can be attached to both simulation and treatment systems. Once the patient is positioned and immobilized in the CT-simulator, the set of coordinates is obtained from the CT-simulator and is transferred to the treatment systems. Often localization lasers and ink/tattoo marks on the patient are used to position the patient on the treatment couch. In addition, restraint and repositioning devices are sometimes used, such as plastic holders for the head, body molds, tilt boards, etc. $^{21}$

The full dose in radiation therapy is delivered in smaller fractions. The biological rationale behind fractionation is to allow normal healthy cells to repair, as normal cells have a greater propensity for repair than tumor cells. ${ }^{17}$ The most common conventional (i.e., non-hypo-fractionated) fractionation scheme for breast treatment is 2 Gy per fraction for five days a week over five weeks.

The clinical evaluation of a treatment plan uses dose-volume histogram (DVH), which graphically summarizes the cumulative dose distribution within a volume of interest. ${ }^{22}$ Various DVH endpoints can be used to compare treatment plans, and DVH objective and constraints are used as clinical endpoints during treatment planning optimization. An ideal cumulative DVH for a target is a step function, where a uniform dose is delivered to the entire volume of the target. ${ }^{17}$ An ideal DVH for an OAR is a delta function at zero dose, where no dose is delivered to the volume. However, these ideal DVHs are not physically achievable. A good target DVH is one where the bulk of the target volume receives the Rx. For example, at least $95 \%$ of the target volume should receive the Rx or more. Similar constraints are applied to OARs, where, for example, no more than 
$50 \%$ of the structure can receive 5 Gy. An example of the target and OAR DVH metrics that will be used in this study are shown in Figure 1.6.

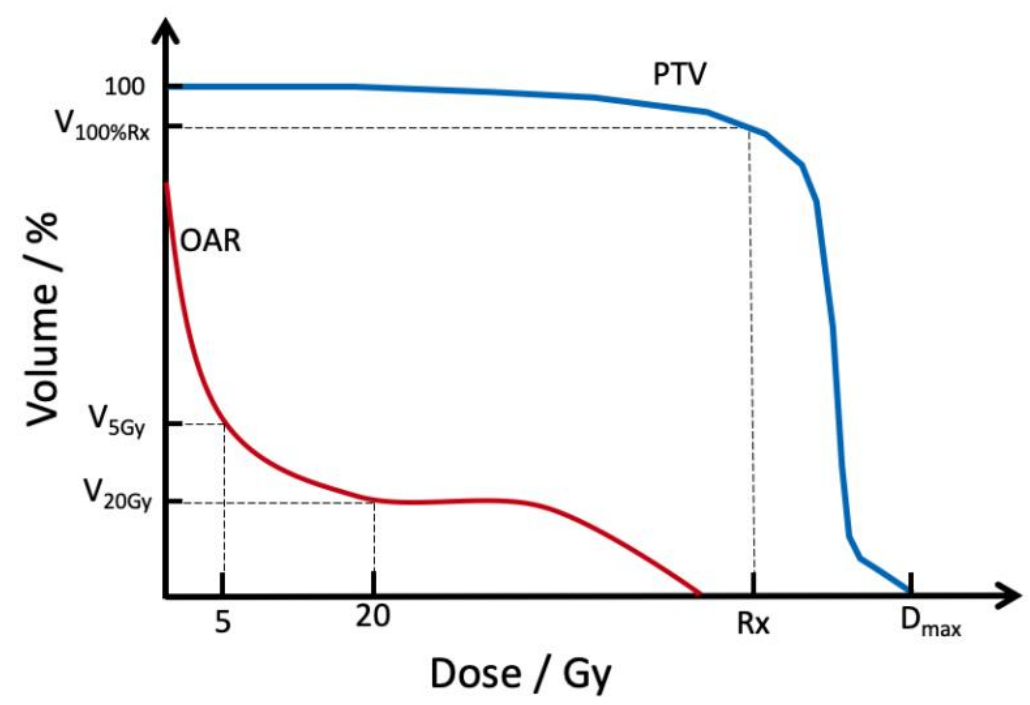

Figure 1.6: An example of a hypothetical DVH curve of a PTV (blue) and an OAR (red) showing the metrics $\mathrm{V}_{100 \% \mathrm{Rx}}$ of the PTV, $\mathrm{V}_{5 \mathrm{~Gy}}$ and $\mathrm{V}_{20 \mathrm{~Gy}}$ of an OAR and $\mathrm{D}_{\max }$ (maximum dose). $\mathrm{V}_{\mathrm{x} \% \mathrm{Rx}}$ is defined as the fractional volume of a structure that receives $x \%$ of $\mathrm{Rx}$. $\mathrm{V}_{\mathrm{xGy}}$ is the fractional volume of a structure that receives $x \mathrm{~Gy}$.

\subsection{Radiation therapy techniques for breast cancer}

The different treatment planning and delivery techniques for the breast include 3D conformal radiation therapy (3DCRT), intensity-modulated radiation therapy (IMRT)based techniques such as Volumetric Modulated Arc Therapy (VMAT), and helical Tomotherapy. Different considerations are factored when determining the optimal delivery technique, such as the anatomy of the patient, target volume, and OAR constraints. The different techniques will be discussed in the following subsections because they are extensively used in this study. The last subsection will describe some of the common commercially available photon dose algorithms that are used for treatment planning with the techniques mentioned above. 


\subsubsection{Three-Dimensional Conformal Radiation Therapy (3DCRT)}

The term 3DCRT refers to the use of 3D patient-specific data, such as the acquired CT scan to create a plan with treatment fields that conform to the target volume. 3DCRT is a forward-planned technique where various treatment parameters are optimized by the planner on a trial-and-error basis. Although forward planning can be very labor-intensive, there are standard techniques and guidelines planners follow for different treatment sites, followed by patient-specific customization.

3DCRT is the most common delivery technique for treating the breast. ${ }^{23}$ The standard radiation field setup consists of two main parallelly opposing fields, also called tangential fields, that are open anteriorly for plan robustness against breathing motion during the treatment and swelling of the breasts over the five weeks of treatment. The tangential fields are angled to avoid the contralateral breast and minimize the dose to the ipsilateral lung and heart. To avoid beam divergence into the lung, the posterior half of the beams is blocked, and a non-divergent match line is formed between the two fields. Multiple smaller subfields are added to achieve a homogenous dose distribution. Figure 1.7a shows a schematic of the setup of tangential fields for a hypothetical breast PTV. One of the greatest benefits of this technique is the ability to deliver dose to the whole breast while sparing the lungs, heart and contralateral breast. 
(a)

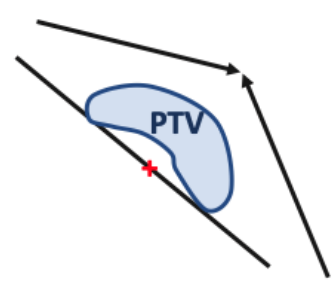

(b)

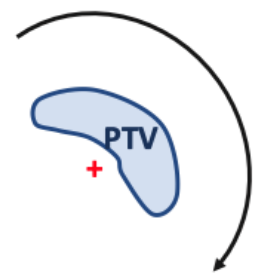

(c)

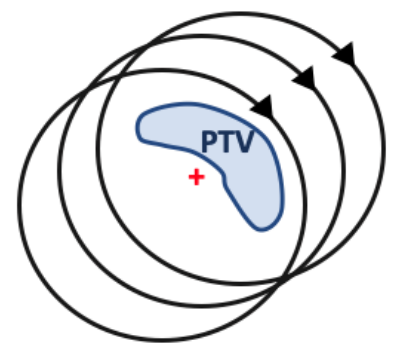

Figure 1.7: Schematic representation of the beam setup for different breast treatment techniques around the PTV. The red cross is the isocenter of the plan. 3DCRT with two tangential fields (a), VMAT with a partial arc around the target (b), and helical Tomotherapy with a rotating fan beam around the target (c).

\subsubsection{Volumetric Modulated Arc Therapy (VMAT)}

IMRT is a radiation delivery technique where non-uniform fluence is delivered to the patient from different directions through a large number of uniform-fluence beamlets. ${ }^{14}$ The beams are optimized to deliver high dose to the target volume while an acceptably low dose is delivered to surrounding normal structures. The most frequently used delivery mechanism of intensity-modulated beams is by a computer-controlled multileaf collimator (MLC) system. The MLC system consists of a large number of leaf pairs (the system used in this study has 80 pairs) of collimating blocks, made of a high atomic number material that are thick enough to block the transmission beam by orders of magnitude from its original intensity. ${ }^{24}$ The MLCs are independently driven to generate arbitrarily shaped fields to conform the delivered dose to the target. The optimization of IMRT plans involves inverse planning where the optimal intensity of each beam and the MLC sequence are determined..$^{25}$ 
VMAT is an-IMRT version of treatment delivery where the gantry rotates continuously with the MLC leaves and dose rate varying throughout, delivering the dose in a contiguous arc. ${ }^{26}$ MLC leaf motion and fluence weights are constrained during optimization such that the delivery sequence is physically achievable. The advantage of VMAT is that the entire treatment can be delivered fast, reducing amount of patient motion during the treatment and allowing for more patient throughput on a given LINAC.

For treating left-sided breast cancer, VMAT was shown to achieve similar PTV coverage while sparing normal structures with less fluence and shorter delivery time than a conventional IMRT plan. ${ }^{27}$ VMAT was also considered advantageous for treating complex chest wall anatomy, by reducing dose to OARs while not compromising the target coverage when compared with 3DCRT plans. ${ }^{28}$ To reduce the dose volume of the contralateral breast and lung, the VMAT arc is often limited to a partial arc such that it covers only the treated breast. ${ }^{29}$ Figure $1.7 \mathrm{~b}$ shows a schematic representation of a typical partial arc setup for a breast VMAT plan.

\subsubsection{Helical Tomotherapy}

Mackie et $\mathrm{al}^{30}$ proposed an IMRT technique where the radiation is delivered as a rotated fan beam which is modulated by a binary MLC system, while the patient is translated through the gantry. The MLCs consist of 64 tall and narrow leaves that are moved pneumatically to either an open or a closed position. Since the gantry continuously rotates around the patient, some areas of the body receive direct low doses, while in a 3DCRT treatment would receive only scatter dose. ${ }^{31}$ In breast treatments, the contralateral breast and lung are of concern. To reduce the dose delivered to these structures, they can be 
defined as completely blocked during treatment planning (i.e., no entrance or exit beams through). Alternatively, these structures can be directionally blocked by allowing the beams exit through the structure, but not enter the structure on their track to the target. Tomotherapy units are also equipped with a megavoltage CT (MVCT) imaging unit used for patient setup verification prior treatment. The MVCT feature was the main enabler of this study, as discussed in section 2.2 .

For breast irradiation, due to the highly modulated beamlets, Tomotherapy can deliver highly conformal doses to target volumes, and was shown to provide better homogeneity than 3DCRT plans, especially when regional lymph nodes were included. ${ }^{32-}$ ${ }^{34}$ Figure $1.7 \mathrm{c}$ shows a schematic of the helical delivery of the beam around the target.

\subsubsection{Dose calculation algorithms}

The most common dose calculation algorithms that are used clinically for treatment planning are Convolution/Superposition (CS)-based algorithms and Monte Carlo (MC). ${ }^{17}$ CS is a model-based approach, where pre-calculated kernels are used to represent the dose at an interaction point. A point dose kernel is defined as the resulted dose distribution in water from scattered photons and secondary electrons. ${ }^{35}$ The kernels are computed by MC simulations. In Collapsed Cone (CC) convolution, an approximation is applied to improve computation time by collapsing the kernels into a discrete number of directions. ${ }^{36} \mathrm{CS}$ is more accurate than $\mathrm{CC}$ in media interfaces, particularly in low density regions, when compared with a reference $\mathrm{MC}$ calculation. ${ }^{36}$

$\mathrm{MC}$ techniques for radiation transport use probability distributions that govern physical processes of particle interactions to simulate the transport of electrons and photons 
in matter. ${ }^{37}$ By simulating a large number of histories, and following the path of individual particles in the patient, the dose to a volume of interest can be determined. MC dose calculation algorithms are computationally more intensive than other techniques, but they offer more accurate results, particularly at extreme tissue interfaces and around high atomic number materials.

It is important to note that the implementation of different dose calculations algorithms is different in the various commercial TPSs, which can lead to gains in speed and various levels of limitations.

\subsection{Literature review}

For patients with $\mathrm{T} 3$ to $\mathrm{T} 4$ breast cancer, postmastectomy radiation therapy has been proven to reduce locoregional recurrence and prolongs survival. ${ }^{38-40}$ However, the timing of the reconstruction still remains controversial for patients who receive postmastectomy radiation therapy. Radiation can cause fibrosis (scarring of the skin), shrinkage and decreases the elasticity of the skin, and can compromise the tissue expansion of patients undergoing a delayed reconstruction. ${ }^{11}$ Therefore, some patients receive radiation treatment with the tissue expander in place. For these treatments, the metal port is frequently, if not always, within the radiation field. The presence of the inhomogeneities, and specifically high-density materials, in the radiation field affect the dose distribution around the inhomogeneity. This has raised the possibility of the metal port affecting the dose distribution within the target volume, which can compromise the outcome of the treatment. 
The dosimetric effect of the static presence of the metal port during radiation therapy has been previously studied, reporting an array of results. Moni et $a 1^{41}$ used thermoluminescent dosimeters (TLDs) to measure the dose around the metal port irradiated with tangential fields and found no increased dose at the surface of the expander. Thompson et al ${ }^{42}$ used p-type diodes to measure the dose distribution around the metal port in a water phantom. They found that in a tangential pair of parallel opposed beams there is a potential underdose of the target by $10 \%$. Damast et al ${ }^{43}$ performed ex-vivo and in-vivo film and TLD measurements of the exit beam from the medial and lateral beams in a tangential treatment. The shadow cast by the metal port can lead to areas of an underdosage of $22 \%$ when irradiated with one $6 \mathrm{MV}$ beam. The effect is less pronounced for a $15 \mathrm{MV}$ beam, where an underdosage of $16 \%$ is measured in film dosimetry both in a phantom and in a real treatment for one patient. The in-vivo TLD measurements resulted in an acceptable variation of the skin dose. Chatzigiannis et a ${ }^{44}$ performed $\mathrm{MC}$ simulations on a patient model to measure the effect of the metal port in $6 \mathrm{MV}$ and $18 \mathrm{MV}$ tangential photon beams. They found a relative dose decrease in the shadow of the metal port of $7 \%-13 \%$ for the $6 \mathrm{MV}$ beams, and a decrease of $6 \%$ for the $18 \mathrm{MV}$ beams. Chen $\mathrm{el} \mathrm{al}^{45}$ found that the presence of the metal port decreased the dose to the target volume by an average of $11.7 \%$. With a short follow-up period of 3 years, they found a local control rate of $91.7 \%$ which did not correlate with the reduced target coverage. Another study evaluated IMRT plans of 41 patients who underwent postmastectomy radiation therapy with the tissue expander in place and found good local control with acceptable doses to heart and lungs. ${ }^{46}$ For their study the 30-month actuarial local control was $97 \%$. Trombetta et al ${ }^{47}$ performed a MC simulation on a phantom with the metal port and measured the effect of the presence of the 
metal heterogeneity in $6 \mathrm{MV}$ photon beams. They found no significant changes in the dose distribution to clinically relevant structures when opposed pair of beams were used. Strang et $\mathrm{al}^{48}$ performed TLD measurements in a torso phantom around the metal port, when irradiated with photons and electrons. No significant dose variation was measured around the metal port for $6 \mathrm{MV}$ tangential beams. When compared with a TPS calculation, no significant difference was measured around the tissue expander implant. Asena et al ${ }^{49}$ used film dosimetry in a breast phantom containing a metal port and irradiated with different beam geometries. They observed an average dose reduction of $20 \%$ from a single tangent $6 \mathrm{MV}$ beam. In a second study by Trombetta et $\mathrm{al}^{50}$, they compared a TPS calculation algorithm to film measurements in a phantom in the presence of the metal port. When considering the real density of the metal port, the TPS calculation showed changes of around $10 \%$ in the isodose curves compared with the film measurements. Gee et al ${ }^{51}$ performed in-vivo film measurements by placing film under the bolus on patients with tissue expanders. An average dose reduction of $7 \%$ was measured to skin in the shadow of the metal port. Park et $\mathrm{al}^{52}$ performed $\mathrm{MC}$ calculations on patients with the metal port and analyzed the dose to OARs and the PTV excluding the metal port. They concluded that no dosimetric changes occur within the PTV and OARs in the presence of the metal port. Da Silva et $\mathrm{al}^{53}$ used an acrylic breast phantom and film measurements to determine the effect of the presence of the metal port in $6 \mathrm{MV}$ tangential photon beams. They found no scattering or attenuation of the beam in areas of the breast that have clinical relevance. Yoon et $\mathrm{al}^{54}$ compared three ways of modelling the metal port in a TPS in different treatment techniques. They concluded that the presence of the metal port has a negligible dosimetric impact on the chest wall, however, a significant dose shadow in the skin is 
present in a conventional tangential plan. This effect is less pronounced in VMAT and four-field IMRT plans. Mizuno et al $^{55}$ used film dosimetry and depth dose measurements in $4 \mathrm{MV}$ and $6 \mathrm{MV}$ photons to establish a method for determining the apparent density to use for the metal port in a given TPS.

Chen et $\mathrm{al}^{45}$ recognized that the metal port has the potential to migrate within a small volume throughout the course of the treatment, for which the TPS does not account. In addition, they noted that daily setup errors and changes to the anatomy of the patient can introduce more uncertainty to the delivered dose. A couple of other groups mentioned the uncertainty associated with contouring the metal port on the TPCT during treatment planning. ${ }^{43,54}$ The high-density metal port induces heavy metal artefacts in the simulation kilovoltage $\mathrm{CT}(\mathrm{kVCT})$, which introduce an uncertainty when trying to locate the metal port. An example of the metal artefacts in the $\mathrm{kVCT}$ is shown in Figure 1.8 for a representative patient with a temporary tissue expander.

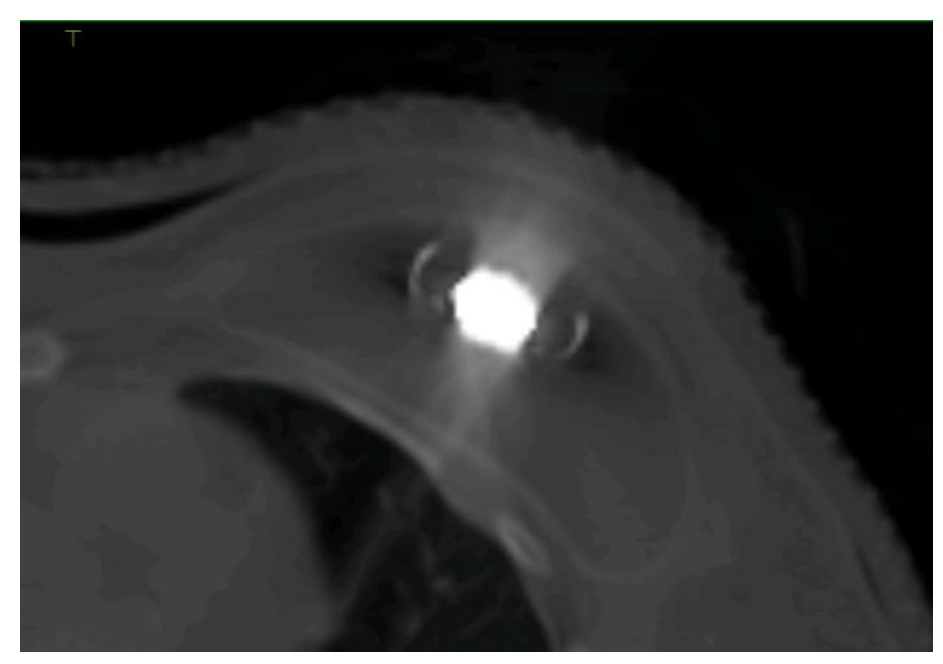

Figure 1.8: An axial kVCT slice of a representative patient with a temporary tissue expander in place, where streak artefacts around the metal port are visible. The window level is adjusted for viewing high-density materials, and yet, the artefacts distort the structure of the metal port. 
The metal streak artefacts are caused by several mechanisms related to the metal itself (e.g., beam hardening and scatter) and to the metal edges. ${ }^{56}$ Metal artefacts are more pronounced with high atomic number metals such as iron, samarium and neodymium, and are less pronounced with low atomic number material such as titanium. This is evident in Figure 1.8 as more artefacts appear around the magnet (samarium) than the titanium shell. These metal artefacts may cause a systematic uncertainty in the position of the metal port if it is contoured incorrectly, as it will not represent the true position of the metal port throughout the course of the treatment.

\subsection{Intra-fraction and inter-fraction motion}

The increased dose conformality of current treatment techniques makes the delivery more vulnerable to different types of uncertainty. These include uncertainties in the image segmentation process, machine-associated uncertainties during treatment deliveries and patient-related uncertainties. If these uncertainties are not accounted for, unintended changes to the delivered dose may compromise the target coverage and overdose OARs. Motion is one class of patient-related uncertainty that is present in the delivery stage of radiation therapy and can be further divided to two types of motion: intra-fraction and interfraction motion. Intra-fraction motion refers to movements that occur during a treatment fraction, such as breathing motion, bowel movements, and sudden patient movements such as coughing, jerking, twitching, etc. Inter-fraction motion refers to changes that occur between treatment fractions, such as different bladder filling, edema (swelling of the 
treatment site), weight fluctuations, etc. Inter-fractional changes also include setup uncertainties of the patient on the treatment couch.

A plan is "robust" when the treatment goal is met even in the presence of uncertainties. The robustness of plans against a certain uncertainty depends on the treatment site, delivery configuration and technique. The robustness of a plan can be evaluated by measuring the relative change of various clinical dosimetric parameters in the presence of these uncertainties. According to the ICRU, changes of $\pm 5 \%$ are clinically acceptable. $^{20}$

\subsection{Objectives of this study}

No study had previously investigated the magnitude and the dosimetric effect of the interfractional positional variation of the metal port. There are three main causes that can contribute to variations in the position of the metal port during daily treatment relative to its reference position in the simulation scan. First, during patient setup and registration before each daily treatment, therapists are often faced with competing considerations and may choose to make compromises in the accuracy of matching the metal port to its reference scan position to achieve an overall better patient setup. Second, anatomical changes to the patient that occur throughout the course of the treatment can alter the shape of the tissue expander and lead to positional variations of the metal port. Examples include body weight changes, breast tissue composition changes, ${ }^{57}$ fibrosis, shrinkage and elasticity changes of the overlaying skin and chest wall tissue. ${ }^{58}$ Third, potential migration of the tissue expander within the submuscular plane leads to port movement relative to the internal anatomy of the patient. The three sources above contribute to positional variability 
between fractions, which can result in unintended dose perturbations within the target volume. These dose perturbations can be clinically relevant because the potential residual microscopic disease after mastectomy is located near the chest wall and the skin, ${ }^{59}$ which directly abut the saline bag of the tissue expander.

The primary objective of this study was to use real patient registration data at the time of treatment to retrospectively determine the magnitude and the dosimetric impact of the inter-fractional positional variations of the metal port. The robustness of the three mostcommon techniques for breast radiation treatment against these inter-fractional variations was quantified and compared. Relative changes in target coverage and doses to relevant OARs were analyzed and compared for each technique when port positional variations were simulated.

\subsection{Thesis organization}

In Chapter 2, the cohort of patients included in this study is introduced, followed by the methods used to measure the inter-fractional positional errors and the results of the measurements for all patients. In Chapter 3 and Chapter 4, the dosimetric effect of the measured errors in different techniques will be quantified. In Chapter 3, the cumulative dosimetric effect of internal port error is investigated, and in Chapter 4 the cumulative dosimetric effect of patient registration errors is examined. Chapter 5 discusses a special case of a single patient who had very large internal port errors due to migrations of the implant within the breast pocket. The dosimetric impact of the measured errors for this patient were retrospectively quantified and compared against other treatment techniques. 


\section{Chapter 2: Measuring inter-fractional positional errors}

In this chapter, the procedure for retrospectively measuring the inter-fractional positional errors of the metal port is described, followed by the results of these measurements for a cohort of patients.

\subsection{Patient population}

Patients who were treated at TOHCC between 2015 and 2017 and had a temporary tissue expander at the time of treatment were identified. This study included six patients who had a mastectomy and underwent an immediate breast reconstruction followed by a radiation treatment while the tissue expander was still in place. The course of the radiation treatment began after the tissue expansion was complete. Three of the six patients had a bilateral mastectomy followed by a bilateral reconstruction. Two of these patients received a bilateral radiation treatment. All patients received 25 fractions to a total Rx of $50 \mathrm{~Gy}$. In this study, each breast treatment was investigated separately resulting in eight independent breast cases and a total of 200 independent fractions. Due to archiving difficulties, out of the 200 fractions, 193 were included in the study. An anonymized list of all the patients included in this study is presented in the appendix.

At TOHCC, patients with tissue expanders are generally treated with 3DCRT. However, in addition to bilateral treatments, patients who have large chest wall curvature are treated on Tomotherapy. The helical delivery in Tomotherapy offers a highly conformal dose distribution to complex anatomies. Figure 2.1 shows the chest wall of two patients 
with tissue expanders. The patient in Figure 2.1a was treated with 3DCRT, whereas the patient in Figure 2.1b was treated on Tomotherapy.
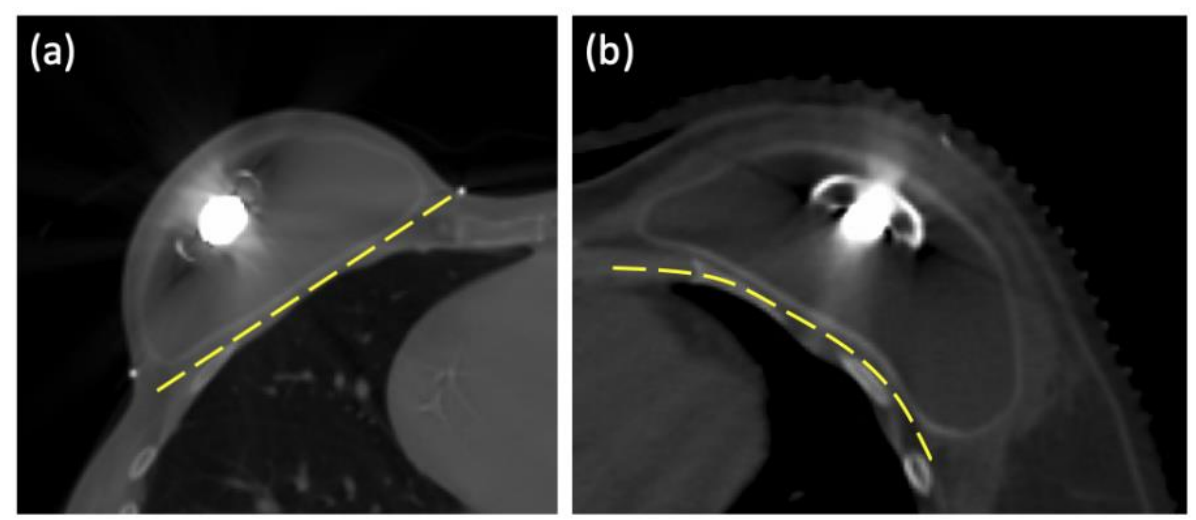

Figure 2.1: An axial slice of the TPCT of two patients with relatively straight chest wall (a), and curved chest wall (b). The patient in panel a was treated with tangential 3DCRT, and the patient in panel $\mathrm{b}$ was treated on Tomotherapy.

\subsection{Patient registration and measuring positional errors}

The accurate setup and positioning of the patient on the treatment couch at every treatment fraction is imperative for a successful outcome of the treatment. Tomotherapy units are equipped with a detector array opposite to the linear accelerator, and the treatment beam can be detuned to become an imaging beam, allowing to acquire an MVCT scan prior the treatment. The MVCT scan is then used to apply minor adjustments in the position of the patient to best align them with the position in the TPCT. This step is referred to as image registration. Tomotherapy allows for corrections in the three cardinal directions (lateral, vertical and longitudinal) and for roll (rotation around the vertical axis), while it cannot correct for pitch (rotation around the lateral axis) or yaw (rotation around the anteriorposterior axis). In order to achieve an accurate image registration, it is crucial to set up the patient on the treatment couch as close as possible to its clinical position before the MVCT 
acquisition. This is achieved with the aid of immobilization devices and by aligning the appropriate localization lasers to the tattoos on the patients and the breast board (device supporting the patient and providing a tilt). Once the patient is positioned on the treatment couch and the MVCT scan is acquired, the MVCT and the TPCT are fused to verify the current positioning of the patient, and that it matches the same position in the TPCT. If necessary, adjustments to the position of patient are made from within Tomotherapy operator's station.

When registering, the therapist checks for specific bone and tissue landmarks for alignment. Priority is given to matching the sternum and ribs at the level of the chest wall PTV, and the spine at the level of nodal PTV. Other anatomical landmarks are the apex of the lungs, the humeral head and the heart. For patients with tissue expanders, the metal port is also a structure that is used for positioning verification. Since anatomical changes in the breast, such as swelling, are expected to occur throughout the course of the treatment, expansion of up $0.5 \mathrm{~cm}$ of the breast is allowed. If couch shifts of over $2.0 \mathrm{~cm}$ and roll correction of over $5^{\circ}$ are required, that indicates that the initial position of the patient was too inaccurate. The patient will have to be physically realigned and the MVCT scan will have to be repeated.

MVCT imaging produces substantially less artefacts than conventional kVCT imaging when high atomic number materials are present in the imaging field of view. The reduced artefacts around the metal port allowed for better localization of the port in the daily MVCT images. At every treatment fraction, after image registration, the MVCT scan after all adjustments is saved in the patients records, co-registered with the TPCT, as seen in Figure 2.2a. This MVCT represents the actual position of the patient at every fraction. 
The Planned Adaptive module of Tomotherapy was used to load the co-registered daily MVCT and the TPCT. This module allows for adaptive re-planning and calculating daily dose distributions based on the daily acquired MVCT. However, for this study, the Planned Adaptive station was only used to view the merged daily MVCT and TPCT. For a given patient fraction, the daily registered MVCT was loaded with the fused TPCT, and the original clinical registration coordinates $(\mathrm{x}, \mathrm{y}, \mathrm{z})$ for that fraction were recorded. The window level of both images was adjusted for an optimal view of the metal port. The 3D registration controls were used to align the metal port on both images, while ignoring the rest of the anatomy. This was done at the three different planes until the metal port was aligned in all directions, and the new registration coordinates were recorded. Figure 2.2 shows two fractions where the metal port matched, and did not match its original position in the TPCT.
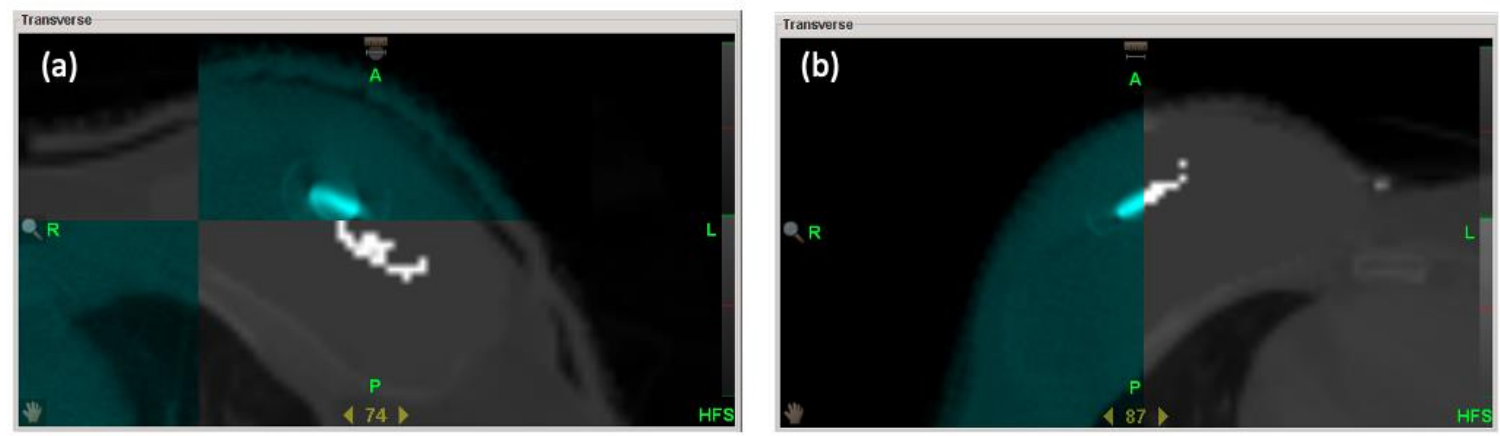

Figure 2.2: Checkered view of an MVCT axial slice in the treatment position (in turquoise) superimposed on the ТРCT (in gray) for two fractions of two different patients. In (a) the metal port is displaced from its original position and in (b) the metal port matches its original position.

The difference between the clinical registration coordinate and the new registration coordinate, where the metal ports was perfectly aligned on both images, represents the 
positional error of the metal port for that fraction. This was repeated for all treatment fractions for each patient. For the patients who had a bilateral treatment, each breast was considered independently.

\subsection{Results: measured inter-fractional positional errors}

Figure 2.3 shows the distribution of the measured inter-fractional positional errors of the metal port for all patients in the three cardinal directions.

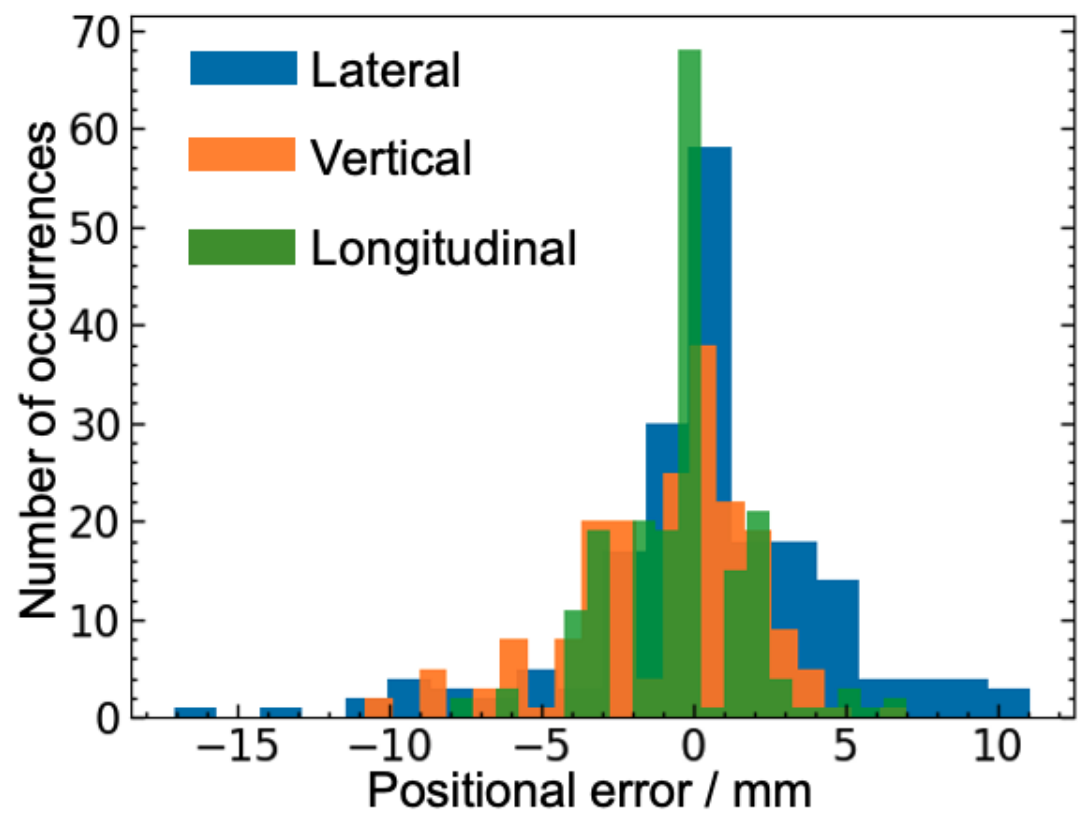

Figure 2.3: Distribution of inter-fractional positional error of the metal port in the lateral (blue), vertical (orange) and longitudinal (green) directions for all patients and fractions $(\mathrm{n}=193)$.

In the lateral direction, positive and negative displacements represent shifts to the left and right sides of the patient, respectively. In the vertical direction, positive and negative displacements represent shifts to the posterior and anterior of the patient, respectively. In 
the longitudinal direction, positive and negative displacements represent shifts to the superior and inferior of the patient, respectively. This notation of directionality will be followed throughout this study.

The measured positional errors in all patients were generally small, with $87 \%$ of the deviations below $5 \mathrm{~mm}$. The positional error in the lateral, vertical and longitudinal directions ranged from -17 to $11 \mathrm{~mm},-10.8$ to $7.0 \mathrm{~mm}$ and -8.0 to $7.0 \mathrm{~mm}$, respectively. Since the roll correction determined by the treating therapists before each treatment fraction was left unchanged in this study when measuring the port positional errors, displacements of the metal port that resulted from roll errors were compensated for with a combination of a vertical and a lateral correction. This explains the skewed distribution of lateral positional errors observed in Figure 2.3.

The positional errors can also be represented per breast case. Figure 2.4 shows the distribution of the measured inter-fractional positional errors of the metal port per breast case in the three cardinal directions. This form of a graph is called a boxplot, where the first and third quartiles of the data are bound by the box. Other elements of the plot include the mean, median and outliers which are represented by the white circle, horizontal line and the asterisks, respectively. In this plot the range of the whiskers extends to the outermost data point that falls within 1.5 times the interquartile range. Similar plots will be used throughout this work. 


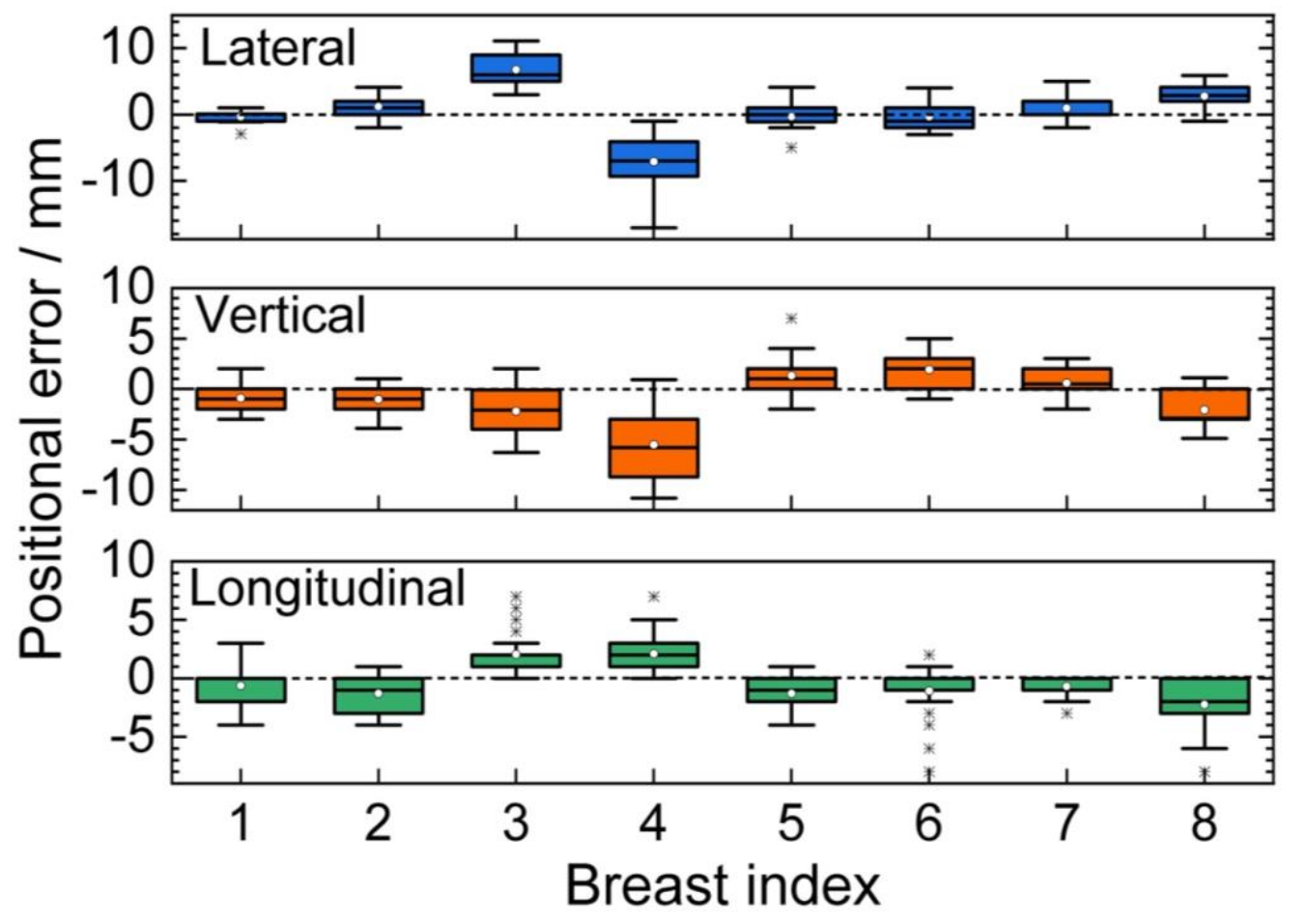

Figure 2.4: Box plots of the distribution of the measured inter-fractional positional variations of the metal port per breast case in the lateral (top), vertical (middle) and longitudinal (bottom) directions.

Patient indices 1 and 2 refer to the right and left breasts, respectively, of one patient who had a bilateral treatment. Patient indices 3 and 4 refer to the right and left breasts, respectively, of one patient with a bilateral treatment. The measured inter-fractional errors for patient indices 3 and 4 (same patient) in the three cardinal directions appear to be systematically not centered around zero. This could indicate that a certain anatomical change in the patient took place after the simulation scan which resulted in the displacement of the metal port in both breasts. This is an example of a systematic port positional error that persisted throughout the course of the treatment. 


\subsection{Summary}

The inter-fractional positional variations of the metal port were retrospectively measured for a cohort of patients previously treated on Tomotherapy. The daily acquired MVCT datasets were used to compare the daily position of the metal port relative to its original position in the TPCT and measure the daily shift of the port. Results show that interfractional positional variations of the metal port were generally small and centered around zero, but errors larger than $1.5 \mathrm{~cm}$ were also be observed. 


\section{Chapter 3: Robustness against internal port positional errors}

In this study, all the factors that can contribute the measured positional errors of the metal port (Chapter 2) are classified from the viewpoint of the metal port into two "classes" of errors. The first class is port positional error relative to the internal anatomy of the patient, referred to herein as internal port error. Internal port error can be caused by anatomical changes of the patient throughout the course of treatment, and/or the migration of the whole tissue expander. The second class is the displacement of the patient relative to the radiation beam, referred to herein as patient registration error. Patient registration error can be caused by necessary compromises made during patient registration, which lead to the whole patient being displaced relative to the radiation beam by the magnitude of the measured error.

The focus of Chapter 3 will be on the modelling and the quantification of the dosimetric effect of internal port error in three treatment delivery techniques. In Chapter 4, the dosimetric effect of patient registration error will be discussed. The first section of Chapter 3 will give an overview of the treatment plans for each of the three radiation therapy techniques. The same treatment plans will also be referred to in Chapter 4, when the modelling of patient registration errors is discussed.

\subsection{Creating the treatment plans}

At TOHCC, immobilization and positioning of patients for breast treatments is identical in tangential 3DCRT, VMAT and Tomotherapy. This is partially attributed to the fact that for some patients the treatment delivery technique is determined only after the simulation scan. 
These patients are scanned and treated supine on a breast board with both arms up. Therefore, the inter-fractional positional errors of the metal port that were measured for the cohort of patients treated on Tomotherapy were assumed to apply to treatments done with tangential 3DCRT and VMAT.

A clinical plan for each of the three techniques was created by a therapist for each of the eight breast cases, for a total of 24 plans. The following are the details of the treatment planning per technique.

For the 3DCRT plans, the commercial TPS Monaco 5.11 (Elekta AB, Sweden) was used to generate the plans. The beam model used was a $6 \mathrm{MV}$ photon beam from an Elekta Synergy LINAC. As discussed in section 1.4.1, the tangential 3DCRT plans consisted of two main parallelly opposing fields that were open anteriorly for plan robustness against breathing motion during the treatment and swelling of the breasts over the five weeks of treatment. Multiple smaller subfields were added to achieve a homogenous dose distribution. No wedges or bolus were used. For patients with bilateral treatments, a separate plan was created for each breast. Monaco limits its dose calculation within the bounds of the patient, defined by the external contour. The dose calculation grid size for the 3DCRT plans was set to $3.0 \mathrm{~mm}$ for all patients, resulting in varying number of voxels per slice, depending on the physical size of each patient. The Monaco implementation at TOHCC has both $\mathrm{CC}$ and $\mathrm{MC}$ as the dose calculation algorithms for 3DCRT plans. CC is the predominant dose calculation engine used clinically for 3DCRT plans, due to its faster calculation speed. However, the CC implementation in Monaco is only accurate for materials with densities of up to $3 \mathrm{~g} / \mathrm{cm}^{3}$. Dose calculations with $\mathrm{MC}$ in Monaco are accurate up to $3 \mathrm{~g} / \mathrm{cm}^{3}$ within the high-density material but the dose deposited to tissue 
immediately adjacent to the high-density material is accurate. This is because for densities of up to $3 \mathrm{~g} / \mathrm{cm}^{3}$ Monaco is able to assign an appropriate tissue composition that corresponds to the voxelized density. For voxels with densities above $3 \mathrm{~g} / \mathrm{cm}^{3}$, Monaco assigns to them the properties of iron, while keeping the true density value. Therefore, although the accuracy of the dose deposition within the high-density material is degraded, the radiation transmission is still accurate.

In this study, $\mathrm{CC}$ and $\mathrm{MC}$ were both used to calculate the tangential 3DCRT plans and were analyzed separately. The MC calculations were performed to $1 \%$ statistical uncertainty per control point (delivered MLC segment). The statistical uncertainty of a plan consists of the individual uncertainties of delivered segments. The exact per-plan uncertainty depends on the weighting and area of overlap of these beams and segments. Therefore, a statistical uncertainty of $1 \%$ per control point corresponds to an absolute statistical uncertainty of approximately $0.7 \%$ of the total dose distribution.

For the VMAT plans, the same version of Monaco TPS and beam model that were used to generate the 3DCRT plans were used. The VMAT plans consisted of a single $230^{\circ}$ arc with the isocenter located in the center of the PTV. Objectives and constraints were set such that the PTV and OARs (such as the lungs and heart) met clinical guidelines. The PTV prescription tolerances were such that D90\% $>50.0$ Gy and D2\% $<55.0$ Gy, where D $90 \%$ is the dose that is received by at least $90 \%$ of the target volume, and D2\% is the near maximum dose that is received by $2 \%$ of the target volume. Meeting the $\mathrm{D} 90 \%$ tolerance ensures that over $90 \%$ of the target receives at least the prescription dose, while the D2\% represents the high-dose region. For the OARs, the dose constraints for the ipsilateral lung were $\mathrm{D}_{\text {mean }}<16 \mathrm{~Gy}, \mathrm{~V} 5 \mathrm{~Gy}<50 \%, \mathrm{~V}_{20 \mathrm{~Gy}}<25 \%$ and $\mathrm{V}_{40 \mathrm{~Gy}}<10 \%$. For the heart, the 
constraints were $\mathrm{D}_{\text {mean }}<8 \mathrm{~Gy}, \mathrm{~V}_{5 \mathrm{~Gy}}<50 \%, \mathrm{~V}_{25 \mathrm{~Gy}}<5 \%$ and $\mathrm{V}_{30 \mathrm{~Gy}}<2.5 \%$. For the VMAT plans, MC-based dose calculation algorithm was used, and the calculation grid size was set to $3.0 \mathrm{~mm}$. Similar to 3DCRT, the VMAT MC dose calculations were performed to $1 \%$ statistical uncertainty per control point.

For helical Tomotherapy, the original clinical plans were used since this cohort of patients was initially treated using this technique. For two of the patients with a bilateral reconstruction, bilateral plans were created, however, each breast was investigated separately. The helical Tomotherapy plans were calculated using the Tomotherapy Planning Station, version 5.1.1.6. (Accuray, CA, USA) which implements CS dose calculation engine. As discussed in section 1.4.3, in Tomotherapy treatments the radiation is delivered helically from all directions, therefore, it is necessary to define blocked structures to prevent the beams from passing through the OARs, such as the contralateral breast and lung. In addition, a modified PTV was defined which excluded the high-density metal port, and the dose objectives for the target were optimized to that modified PTV. The clinical rationale behind this approach is to reduce the fluence weights that would have been delivered to make up for the metal port. Objectives and constraints of the target and OARs were identical to those in the VMAT plans.

Unlike the Monaco TPS, Tomotherapy TPS does not limit its dose calculation to the patient only and calculates the dose outside the patient for a fixed calculation grid. All helical Tomotherapy dose calculation grids were 256 by 256 pixels with a pixel spacing of $2.3438 \mathrm{~mm}$. In the longitudinal direction (superior to inferior), the pixel spacing was equivalent to the slice thickness of the TPCT, which was $3.0 \mathrm{~mm}$. 
The calculation times of the plans varied and are worth noting. The longest calculation time was for the VMAT plans (MC to $1 \%$ uncertainty per control point) which ranged from 40 minutes to over an hour, depending on the patient. The 3DCRT plans calculated with $\mathrm{MC}$ were significantly shorter and came up to $\sim 5$ minutes per calculation. The 3DCRT plans calculated with CC, and the helical Tomotherapy plans calculated with CS dose engines had the shortest calculation times of less than a minute, with the CC-based calculations being faster than the Tomotherapy CS.

\subsection{Modelling internal port positional errors}

The first class of error modelled was internal port error, which is the displacement of the metal port relative to the internal anatomy of the patient. To simulate a displacement of the metal port, for every fraction in each patient the TPCT was modified such that the metal port is artificially shifted by the measured magnitude of the positional error for that fraction. An anonymized TPCT of each patient was exported from the TPS in a DICOM format. DICOM (Digital Imaging and Communications in Medicine) is the international standard format for imaging information, and is the format used to process and view images and dose files. ${ }^{60}$

The first step was to isolate the components of the metal port in the TPCT by identifying which voxels of the CT set belonged to the magnet and the titanium shell. The initial approach was to use thresholding of $\mathrm{HU}$ values that correspond to the physical densities of the components of the metal port. However, there were two main problems with this approach. First, the metal artefacts around the metal port included some dark streaks (areas of low HU values) and bright streaks which distorted the real shape of the 
magnetic core. Secondly, it was difficult to differentiate between voxels that were part of the magnet and the titanium shell based on one thresholding value. Figure 3.1 shows a TPCT slice with the metal port with three different thresholds applied. Voxels with HU values greater than the threshold are colored green. Although the magnetic core could be isolated with a single threshold value, parts of the titanium shell that are close to the magnetic core were masked with dark streaks.

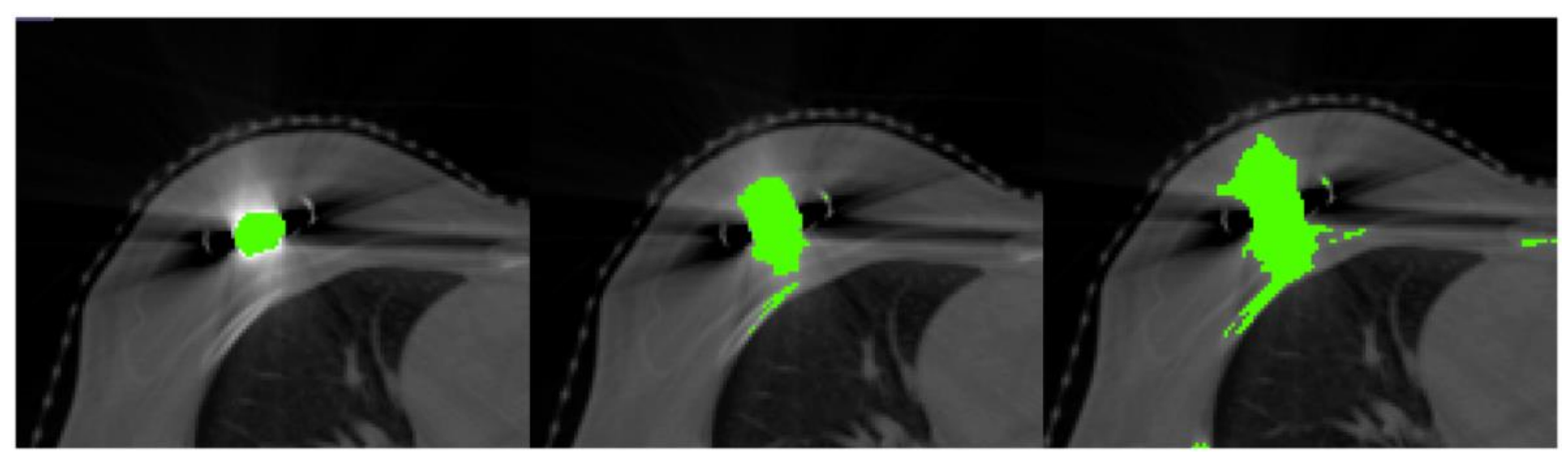

Figure 3.1: TPCT slice of a representative patient with three HU thresholds applied, going from highest threshold (left) to lowest (right). Pixels with HU greater than the threshold are colored green. Lowering the threshold value does not differentiate between the titanium shell and the tissue around due to the presence of heavy metal artefacts.

The second approach to isolating the components of the metal port was to use the structures defined by the radiation oncologists and the treatment planners during image segmentation. Along with the TPCT, the structure set of each patient was exported in a DICOM format. In a DICOM structure set, contoured structures are represented by a set of three-dimensional coordinate points at the bounds of the contour in the CT set.

In-house software was developed using Pydicom to identify voxels in the TPCT that belong to a structure by using the structure set coordinate points (Pydicom is a python package that was used to read and write DICOM files, and was used extensively in this 
study. $)^{61} \mathrm{~A}$ voxel was considered to belong to a structure if the midpoint coordinate of that voxel was enclosed within the contours of that structure. This logic is illustrated in Figure 3.2 , showing the contours of the titanium shell and the magnet for a given slice, where every blue point represents a center of a voxel.

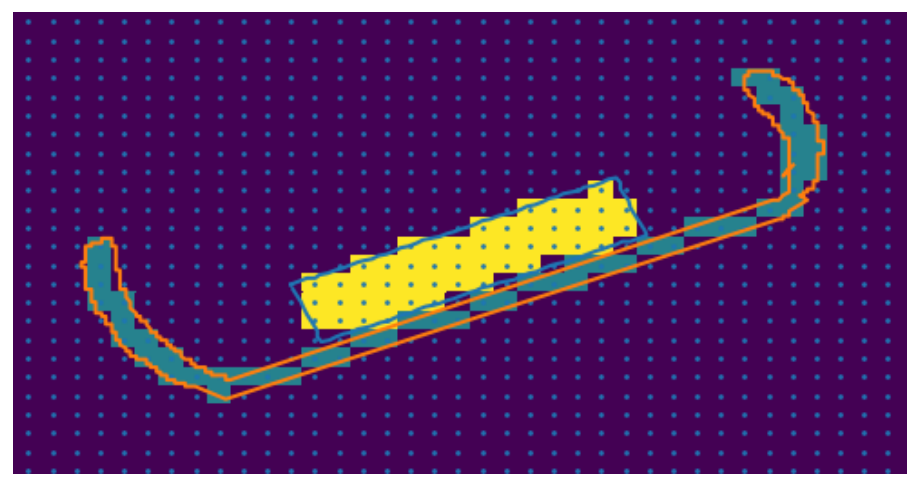

Figure 3.2: Identifying the voxels that belong to the metal port using the clinically defined contours. Blue points represent the center of each voxel. If the midpoint of a voxels falls within the contour, the voxel belongs to the structure. Yellow voxels represent the magnetic component and the turquoise voxels represent the titanium shell.

To correct for the metal artefacts, voxels that were inside the titanium shell and the magnet were assigned $\mathrm{HU}$ values of 3926 and 10248, corresponding to physical densities of $4.0 \mathrm{~g} / \mathrm{cm}^{3}$ and $8.0 \mathrm{~g} / \mathrm{cm}^{3}$, respectively. These physical density values are used clinically in Tomotherapy treatment plans, and thus, were used in this study. The corresponding HU values were derived from the image value-to-density table (IVDT) of the Tomotherapy unit. Since the corrected CT datasets were also used for calculations in Monaco, the relative electron density (RED) of the metal port was found, as Monaco uses electron densities as opposed to physical densities for its dose calculations. The RED values of the titanium shell and core magnet were found to be 2.7617 and 5.2783, respectively. These values were derived from the RED curve of the Monaco TPS. 
The metal artefacts around the metal port were also corrected by assigning the breast the mean HU value of nearby breast tissue that is free from artefacts. An example of a corrected CT slice is shown in Figure 3.3b, where Figure 3.3a shows the same slice before any density overrides. Once the density overrides were applied, the metal port was artificially shifted by the measured inter-fractional error measured in a given fraction, as illustrated by the silhouette in Figure 3.3b. It is important to note that the displacement of the metal port was restricted by the voxel dimensions of the TPCT. In this study the TPCT voxel size was $1.1719 \times 1.1719 \times 3.0 \mathrm{~mm}^{3}$, meaning that the measured displacements in any direction were rounded to the nearest value that is a multiple of the voxel size in that dimension. These steps were repeated for all fractions in each patient, resulting in an independent CT set for every fraction, where the metal port is displaced by the measured port error in that fraction. 


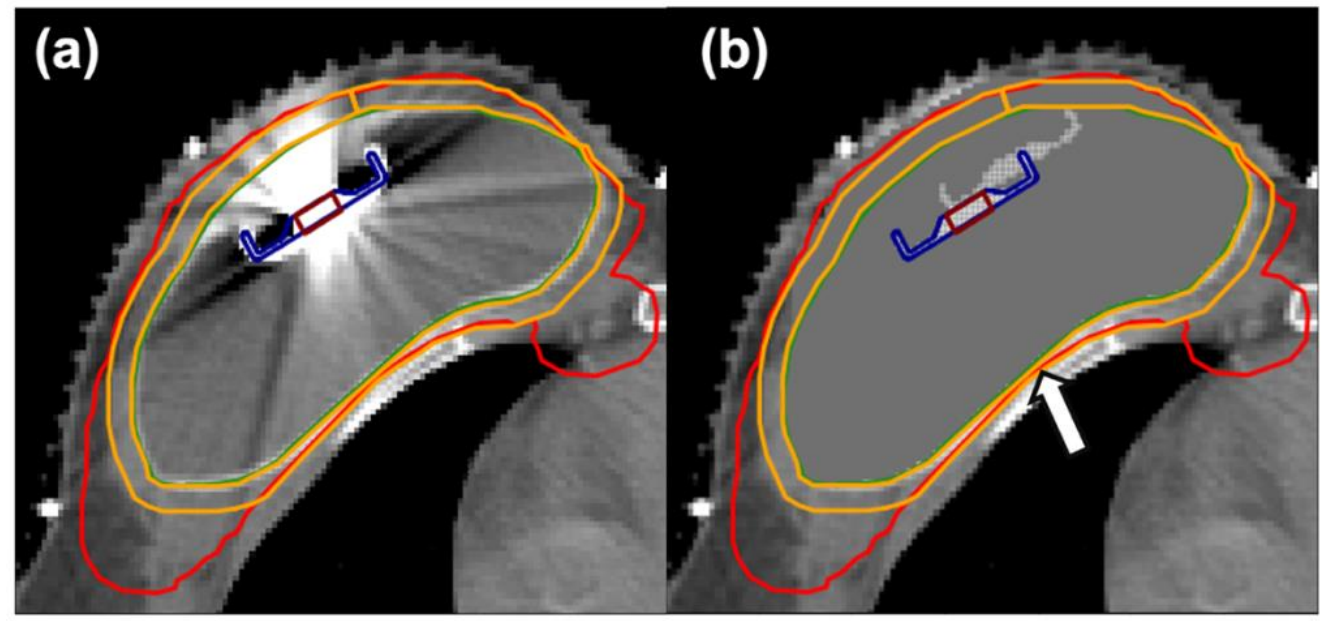

Figure 3.3: Transverse view of a CT slice for a representative patient with a tissue expander before (a) and after (b) density corrections of the temporary implant, the surrounding tissue, and the metal port. The maroon contour is the magnetic metal port, the blue contour is the titanium shell of the port, the green contour is the saline implant, and the red contour is the PTV. The bulge in the lower right of the PTV is the internal mammary lymph node that was included in the PTV. The volume bound the orange contour is the ROI (defined in section 3.3). Note that the ROI is purposely truncated in regions where the $5 \mathrm{~mm}$ expansion extends outside the PTV (see arrow). The non-contoured silhouette of the metal port and titanium shell in panel $\mathrm{b}$ represents a simulated internal port error.

\subsection{Dose calculations and data analysis}

The TPSs Monaco and Tomotherapy have a Data Quality Assurance (DQA) feature which allows calculating clinical plans on imported phantoms for plan checking purposes. Since all modifications to the original TPCT were completed outside of the TPS, these CT sets were imported back to the TPS, and the plans were calculated on the modified CT sets as DQA calculations. The resultant dose distributions were then exported in DICOM formats and analyzed outside of the TPS.

Local dose perturbations in the chest wall and skin can be masked if the breast PTV is used for the analysis because that PTV contains the large non-biological saline bag. For 
more meaningful dose analysis, a Region of Interest (ROI) within the PTV was defined as a $5 \mathrm{~mm}$ expansion around the saline bag which excluded the non-biological bag and its contents, and excluded the metal port. The newly defined ROI included clinically relevant tissue that may contain microscopic disease and is most vulnerable to being affected by the presence of the metal port and its positional errors. ${ }^{59}$ This ROI is shown in Figure 3.3 bound by the orange contour.

The ROI was created in Monaco TPS, using its image segmentation and contouring tools. First, the saline implant was contoured in the slices where the metal port is visible, with additional two slices in the superior and inferior directions. The contour line was drawn in the middle of the bright border that is seen between the implant and surrounding tissue, to ensure that ingrown tissue on the surface of the textured implant was included in the ROI. A second structure was defined by applying a margin around the implant of $5 \mathrm{~mm}$ and clipping it within the bounds of the PTV. The ROI was then defined as the intersection of these two structures, forming a ring-like structure. As pointed by the arrow in Figure $3.3 \mathrm{~b}$, the ROI becomes purposely truncated in regions where the $5 \mathrm{~mm}$ expansion extends outside the PTV.

Clinically relevant DVH metrics for the newly defined ROI and the neighbouring OARs were used to compare the cumulative dose distributions of the investigated scenarios to the originally planned dose distribution. For the ROI, the percent volume that received $100 \%$ of Rx (V100\%Rx) was assessed. For the OARs, two clinically used DVH endpoints were chosen to assess the impact on the ipsilateral lung and the heart. For the ipsilateral lung, the percent volume receiving 20 Gy was assessed ( $\left.V_{20 G y}\right)$ and for the heart, the percent volume receiving $5 \mathrm{~Gy}\left(\mathrm{~V}_{5 \mathrm{~Gy}}\right)$ was assessed. The analysis of the OARs was limited 
to the slices where the ROI was present. Although the metal port has the potential to affect OARs on slices beyond the ROI, limiting the analysis to the slices where the ROI is present allows for a more consistent dose-volume analysis across patients.

\subsection{Results: dosimetric effect of daily measured errors and a systematic error}

For all eight breast cases, the dosimetric effects of internal port errors in each of the three treatment techniques (tangential 3DCRT, VMAT and helical Tomotherapy) were evaluated for two scenarios. The first scenario was the cumulative effect of the daily measured error, referred to herein as "daily variable" error. The second scenario was the cumulative effect of a systematic error, referred to herein as a "systematic" error.

For the first scenario of daily variable error, each plan was recalculated on each of the 25 modified CT datasets per patient. An in-house software developed for this study was used to read the 3D dose data from individual DICOM dose files and perform a dose accumulation of the dose distributions of each simulated fraction through voxel-to-voxel summation. The cumulative dose distribution was then compared with the original planned dose (original plan calculated on a modified CT set with the metal port in its original position) for each plan, and percent dose difference maps normalized to Rx were generated. Figure 3.4 shows for a representative patient the original dose distribution of each of the three techniques overlaid on the corrected TPCT with the metal port located in its original position. Note that for the tangential 3DCRT plan, both $\mathrm{MC}$ and $\mathrm{CC}$ dose calculations are shown. Figure 3.5 shows the percent dose difference map for the same representative patient, relative to the original dose distribution when daily measured internal port errors were modelled in the three planning techniques. 


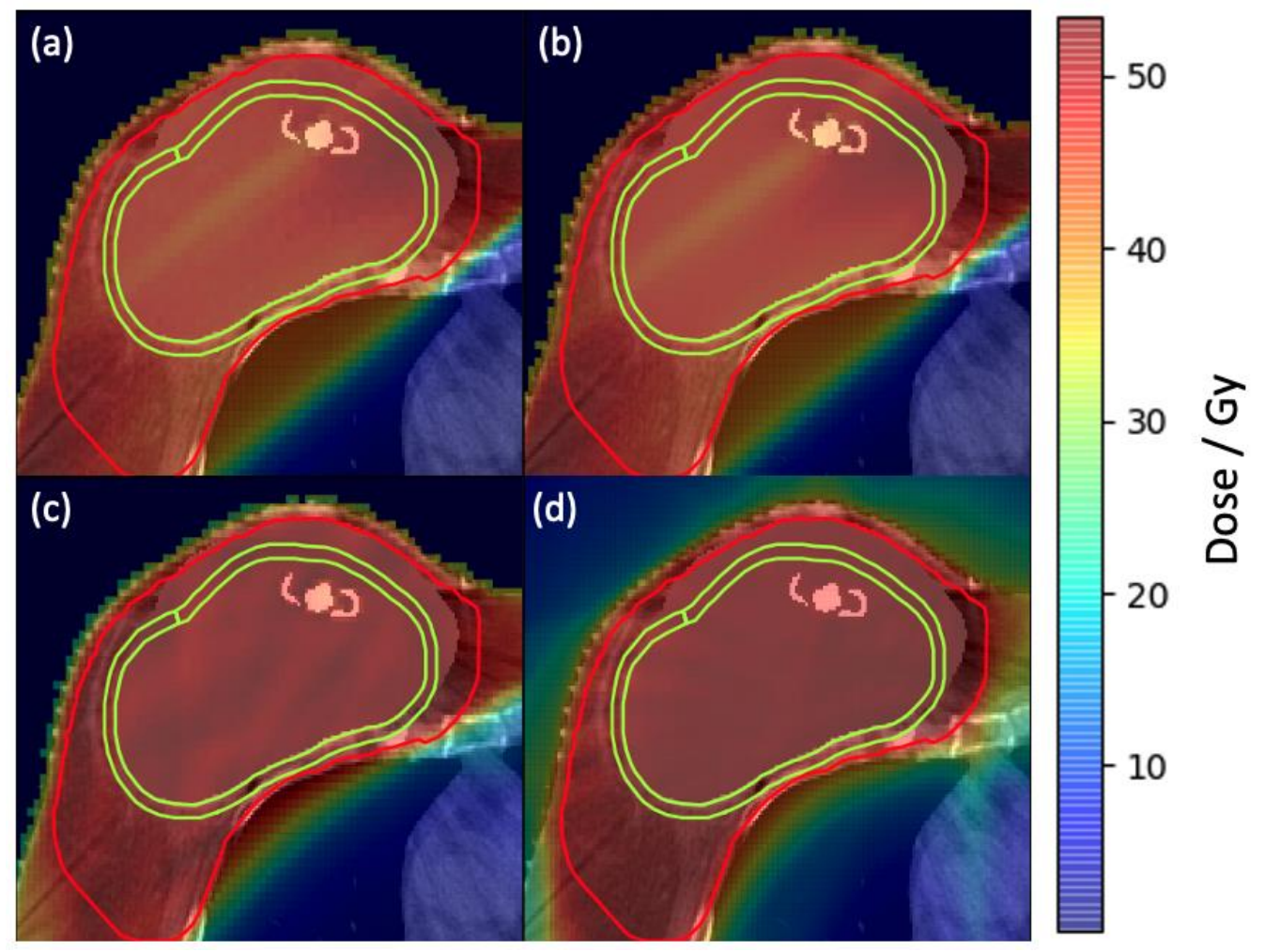

Figure 3.4: Representative axial slice of a representative patient showing the originally planned dose distribution for 3DCRT MC (a), 3DCRT CC (b), VMAT MC (c) and helical Tomotherapy CS (d) treatment techniques. Rx was 50 Gy. The red contour is the PTV and the area bound by the yellow contour is the ROI defined for this study. MC: Monte Carlo, CC: Collapsed Cone, CS: Convolution/Superposition. 


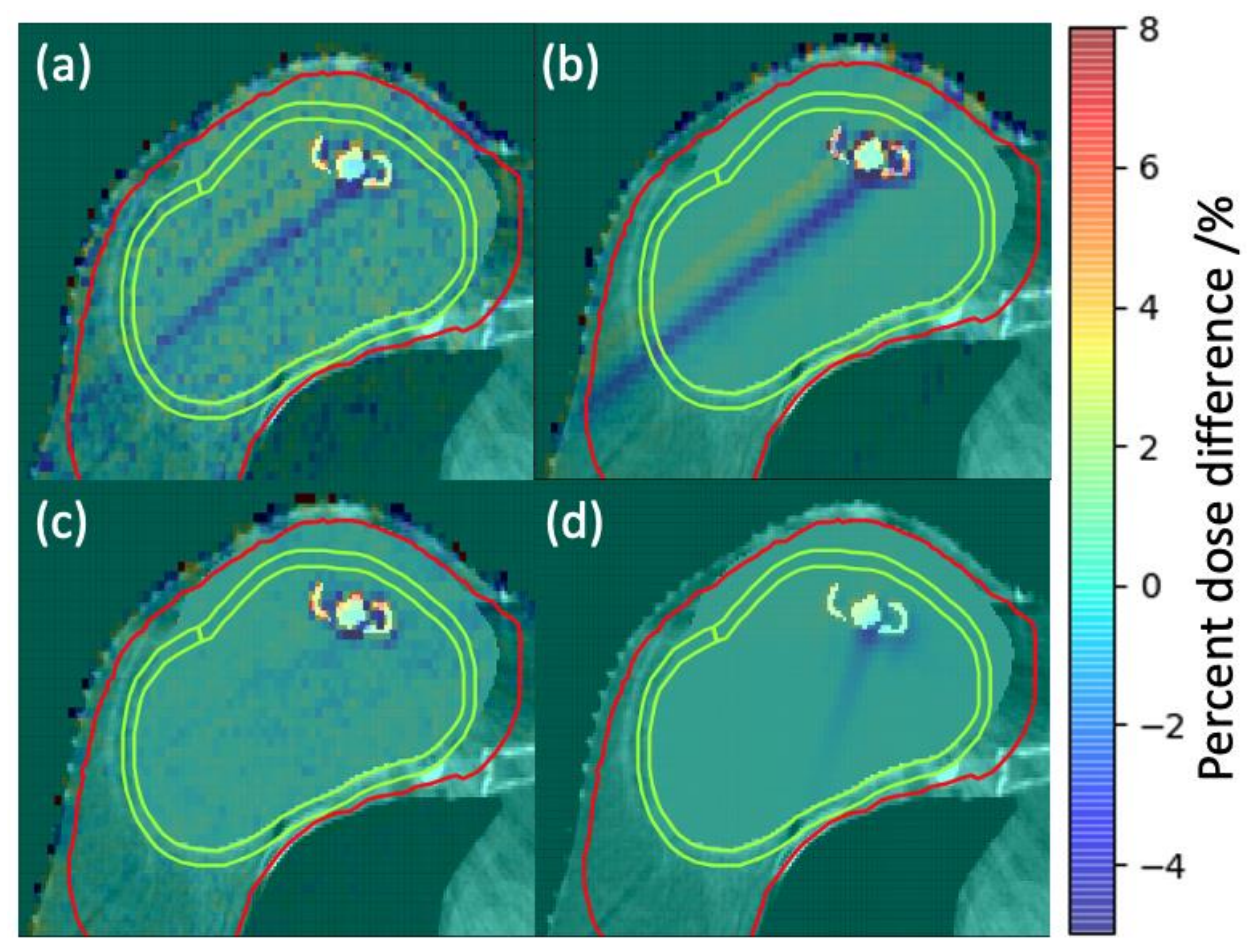

Figure 3.5: Percent dose difference maps for a representative slice and patient when daily variable internal port errors were modelled in 3DCRT MC (a), 3DCRT CC (b), VMAT MC (c) and helical Tomotherapy CS (d). All percent dose differences were normalized to Rx. The contours are the same as in Figure 3.4. MC: Monte Carlo, CC: Collapsed Cone, CS: Convolution/Superposition.

The second scenario investigated was the cumulative effect of a systematic realistic large error in port position. The magnitude of the systematic error was derived from the largest positional error measured during metal port registration. The same magnitude of error was simulated in all patients, however, the direction of the shift was determined on a patient basis, to ensure a realistic position of the metal port. This scenario represents a change in port position after the simulation CT scan was acquired that persisted throughout the course of the treatment. For this scenario, the original plan was calculated on one modified CT dataset, where the metal port is displaced by a large shift. Using the in-house software, the 3D dose data was then multiplied by the number of fractions to represent a 
full course of treatment and was compared with the originally planned dose distribution. The percent dose difference relative to the originally planned dose was computed for each of the three techniques and is shown in Figure 3.6 for the same representative patient.

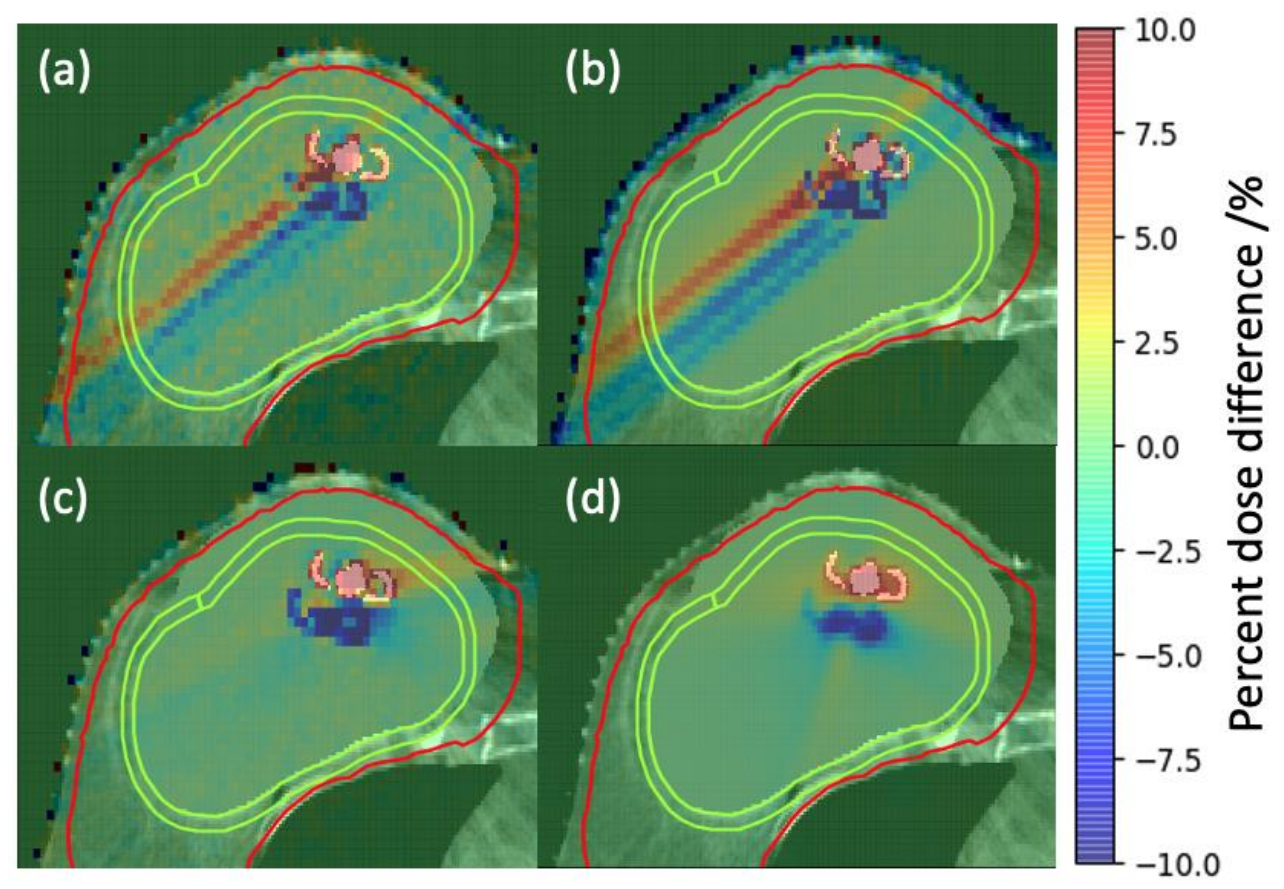

Figure 3.6: Percent dose difference maps for a representative slice and patient when a systematic internal port error was modelled in 3DCRT MC (a), 3DCRT CC (b), VMAT MC (c) and helical Tomotherapy CS (d). All percent dose differences were normalized to Rx. MC: Monte Carlo, CC: Collapsed Cone, CS: Convolution/Superposition.

From the dose difference maps (Figure 3.5 and Figure 3.6), it is observed that an area of underdosage can occur in the shadow of the metal port when the daily measured and systematic port positional errors are modelled. The underdosed area was mainly contained within the non-biological implant in the VMAT and helical Tomotherapy plans. However, there was dose reduction in the skin in the shadow of the metal port that is outside 
the non-biological saline implant in the tangential 3DCRT plans. The underdose are in the skin can be clinically significant because the skin and the chest wall are the possible location for subcutaneous and chest wall recurrences. ${ }^{59}$

From an analysis of all patients, the percent point dose differences inside the ROI when daily variable and systematic internal port errors were modelled in the three techniques is summarized in the boxplot in Figure 3.7. One distinction in this boxplot is that it shows the $1 \%$ to $99 \%$ range denoted by stars, as opposed to the outliers in the previous boxplots.

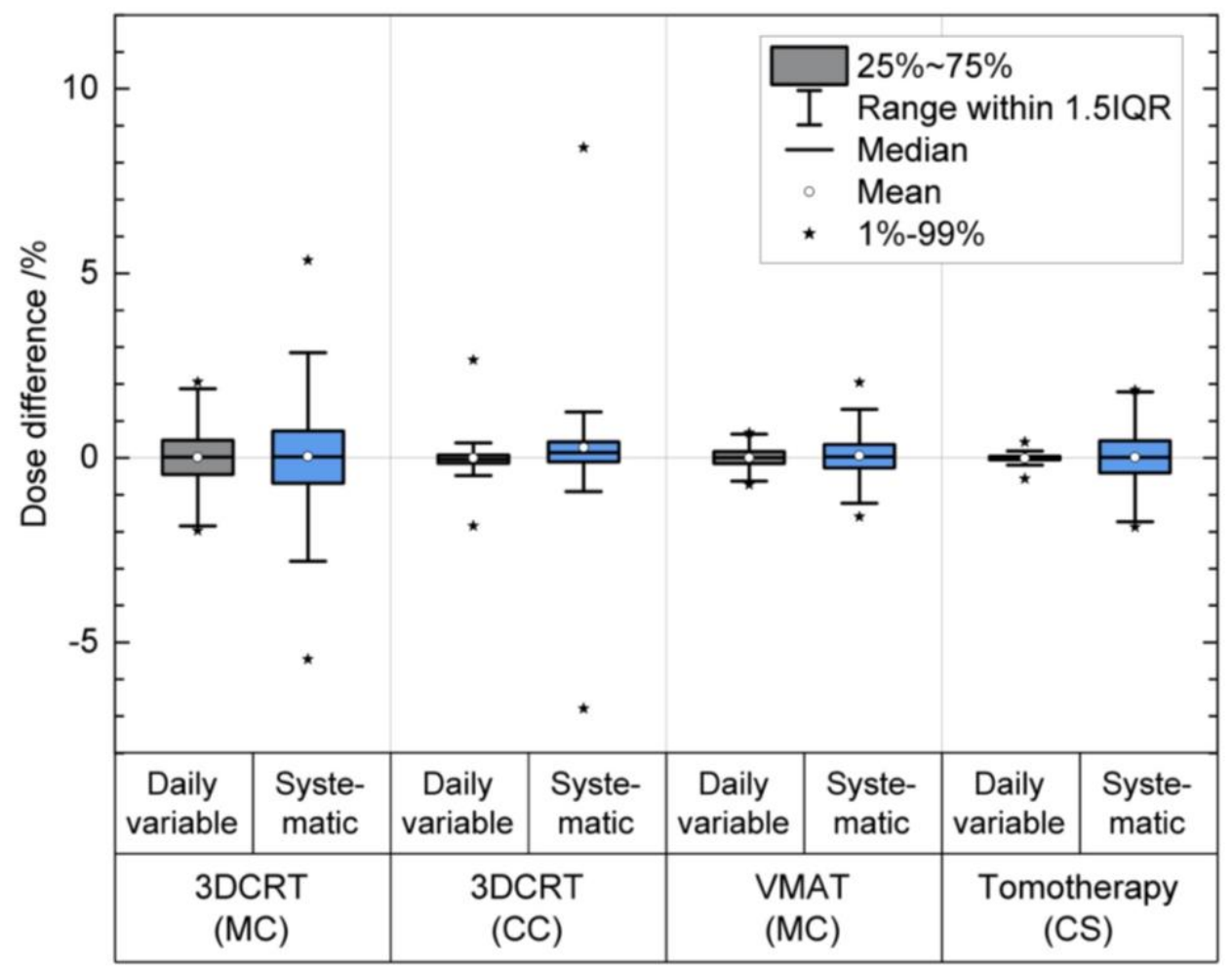

Figure 3.7: Percent point dose differences inside the ROI for all patients when internal port positional errors were modelled in the three treatment techniques (tangential 3DCRT, VMAT and helical Tomotherapy). The modelled daily variable errors are in gray and systematic errors are in blue. All point dose differences were normalized to Rx. The dose calculation engine used with each treatment technique is shown in brackets MC: Monte Carlo, CC: Collapsed Cone, CS: Convolution/Superposition. 
Systematic internal port errors resulted in higher point dose variability than the daily measured error in all three techniques. The cumulative effect of the daily variable and systematic internal port errors resulted in an absolute mean point dose differences close to zero, with the interquartile range being within $1 \%$ in all three techniques. However, the $1 \%$ to $99 \%$ ranges for daily variable port errors were within $2 \%$ for 3DCRT (MC), $3 \%$ for 3DCRT (CC), 1\% for VMAT (MC) and $<1 \%$ for Tomotherapy (CS) treatment plans. For a systematic port error, the $1 \%$ to $99 \%$ range in point dose differences were within $6 \%$ for 3DCRT (MC), 9\% for 3DCRT (CC), 2\% for VMAT (MC) and 2\% for Tomotherapy (CS). The high variability in point dose differences in the 3DCRT plans is seen in the dose difference maps in Figure 3.5 (panels a,b) and Figure 3.6 (panels a,b), where in a given slice, parts of the ROI were overdosed and some areas that fall under the shadow of the metal port were underdosed.

Point dose differences in biologically relevant areas are insufficient for a complete dosimetric evaluation of the impact of internal port positional errors. The DVH (introduced in section 1.4) of the ROI is shown in Figure 3.8 for a representative patient in each of the three techniques. The DVH curve of the original plan (solid orange) is compared against the dose accumulation taking into account the daily variable internal port error (green) and a systematic positional error (blue). From this curve, the $\mathrm{V}_{100 \% \mathrm{Rx}}$ (volume of the ROI receiving $\mathrm{Rx}$ ) was determined for each modelled scenario. 


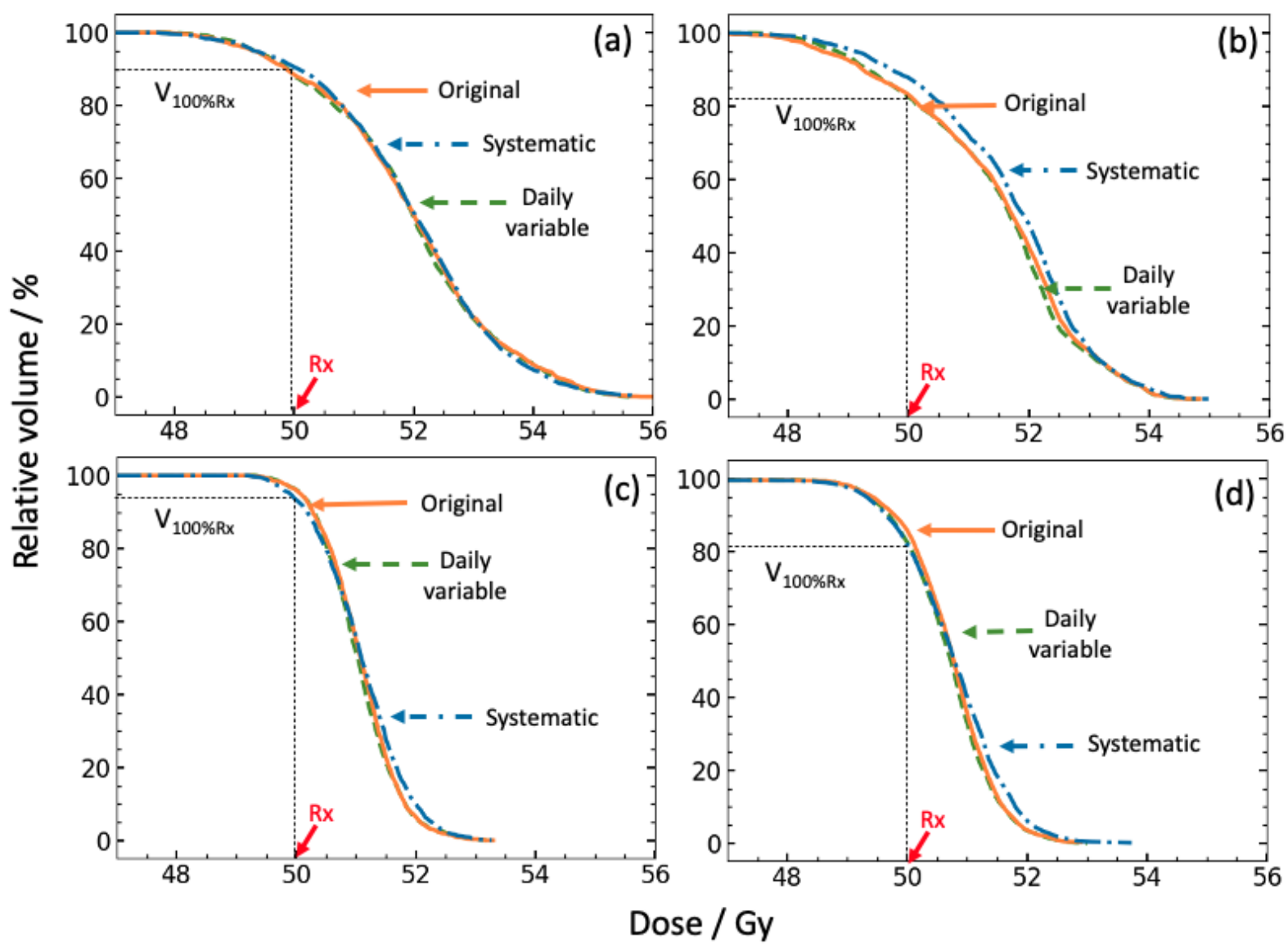

Figure 3.8: DVH of the ROI for a representative patient when internal port positional errors were modelled in 3DCRT MC (a), 3DCRT CC (b), VMAT MC (c) and helical Tomotherapy CS (d). Solid orange: original plan. Green dashed: cumulative dose with daily variable port error modelled. Blue dash-dotted: cumulative dose with a systematic port displacement modelled.

Similar DVH curves were generated for all patients and the change in $\mathrm{V}_{100 \% \mathrm{Rx}}$ of the ROI was calculated relative to the original plan when daily variable and systematic internal port positional errors were modelled. The change in $\mathrm{V}_{100 \% \mathrm{Rx}}$ of the ROI is summarized for all patients in the boxplot in Figure 3.9. For the DVH metrics, the overall range of data points is provided when relevant, as opposed to the $1 \%-99 \%$ range provided in the results previously. 


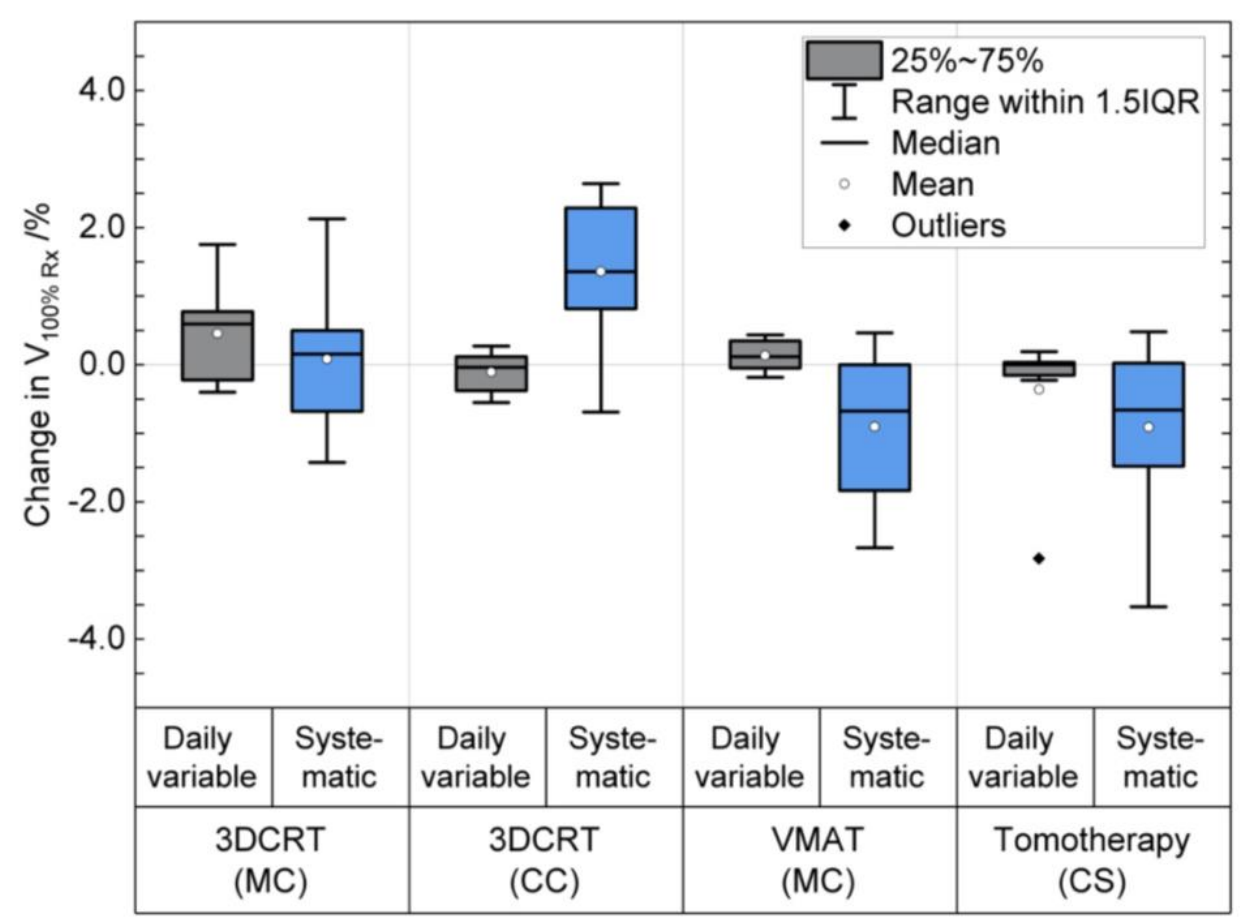

Figure 3.9: The change in $\mathrm{V}_{100 \% \mathrm{Rx}}$ (volume receiving $100 \%$ of $\mathrm{Rx}$ ) of the ROI for all patients when internal port errors were modelled in the three treatment techniques (tangential 3DCRT, VMAT and helical Tomotherapy). The modelled daily variable errors are in gray and systematic errors are in blue. The dose calculation engine used with each treatment technique is shown in brackets - MC: Monte Carlo, CC: Collapsed Cone, CS: Convolution/Superposition.

The cumulative effect of the daily variable internal port errors resulted in absolute mean changes of $<0.5 \%$ in the three treatment techniques, with total ranges of $-0.4 \%$ to $1.8 \%$ for $3 \mathrm{DCRT}$ (MC), $-0.6 \%$ to $0.3 \%$ for $3 \mathrm{DCRT}$ (CC), $-0.2 \%$ to $0.4 \%$ for VMAT and $-2.8 \%$ to $0.2 \%$ for Tomotherapy. The cumulative effect of a systematic internal port error resulted in a mean change of $0.1 \%$ (range: $-1.4 \%$ to $2.1 \%$ ) for 3DCRT (MC), $2 \%$ (range: $-0.7 \%$ to $2.6 \%$ ) for $3 \mathrm{DCRT}(\mathrm{CC}),-0.8 \%$ (range: $-2.7 \%$ to $0.5 \%$ ) for VMAT (MC) and $-1 \%$ (range: $-3.5 \%$ to $0.5 \%$ ) for Tomotherapy (CS). 
All changes in $\mathrm{V}_{100 \% \mathrm{Rx}}$ were small and within the clinically acceptable limits. ${ }^{20}$ The target coverage was affected to a larger extent when a systematic error was present in VMAT and helical Tomotherapy plans compared with the tangential 3DCRT plan. Yet, Figure 3.7 shows that point dose variability inside the ROI can be higher than $5 \%$ in the tangential beam configuration, decreasing the uniformity of the dose distribution within the target.

For the relevant OARs, the DVH of the heart and the ipsilateral lung for a representative patient are shown in Figure 3.10 for each of the three techniques. The cumulative effect of daily variable and systematic internal port errors on the OARs were very small, therefore, the DVH curves of the modelled scenarios almost perfectly overlap. Similar DVH curves of the OARs were generated for all patients and the change in $\mathrm{V}_{20 \mathrm{~Gy}}$ of the ipsilateral lung and $V_{5 \mathrm{~Gy}}$ of the heart were calculated for the modelled scenarios, summarized in the boxplots in Figure 3.11 and Figure 3.12, respectively. 


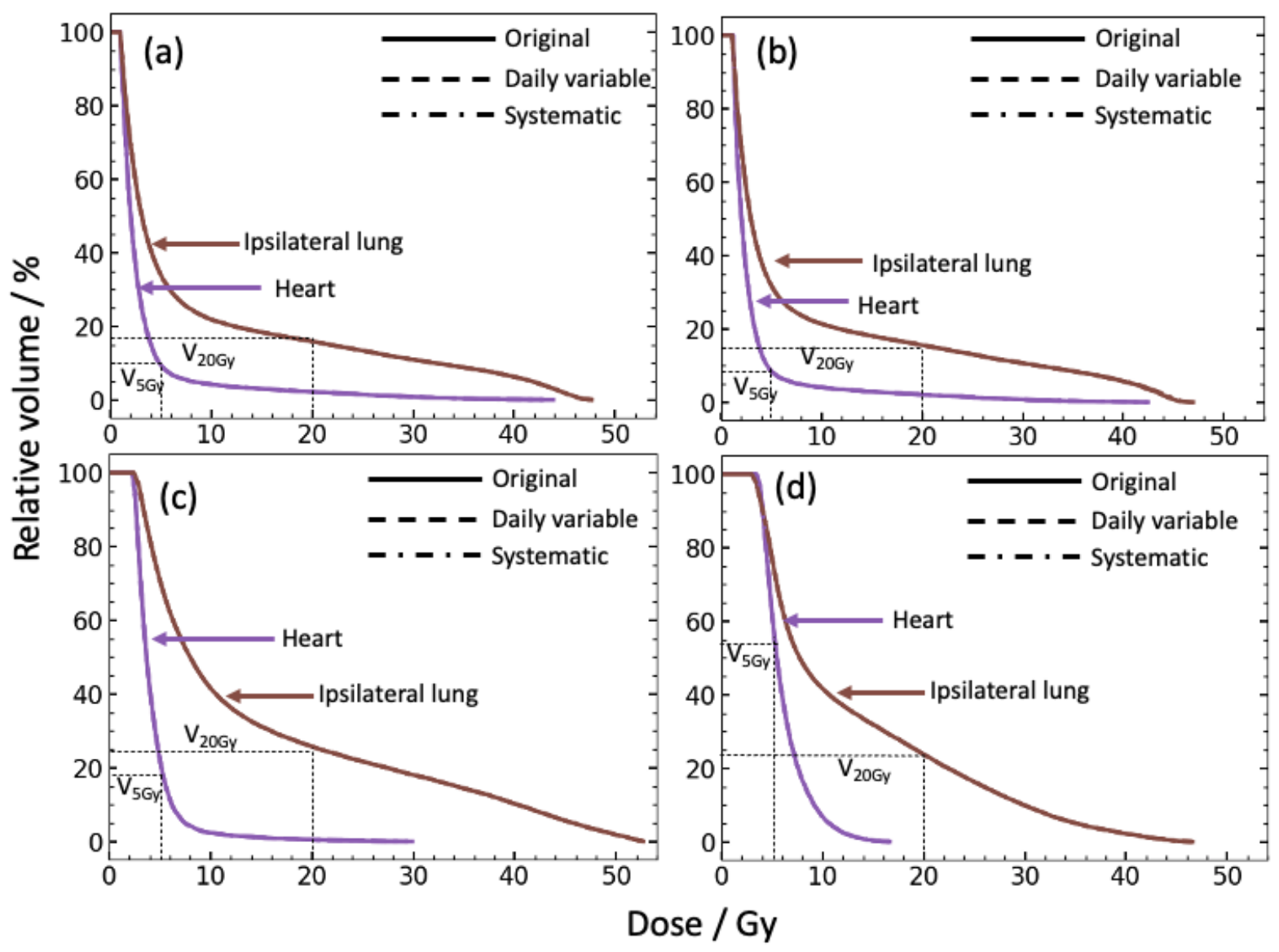

Figure 3.10: DVH of the ipsilateral lung (in brown) and the heart (in purple) for a representative patient when internal port positional errors were modelled in 3DCRT MC (a), 3DCRT CC (b), VMAT MC (c) and helical Tomotherapy CS (d). Solid: original plan. Dashed: daily variable error modelled (superimposed under the solid line). Dash-dotted: systematic error modelled (superimposed under the solid line). The metrics $V_{20 G y}$ of the lung and $\mathrm{V}_{5 \mathrm{~Gy}}$ of the heart are shown. 


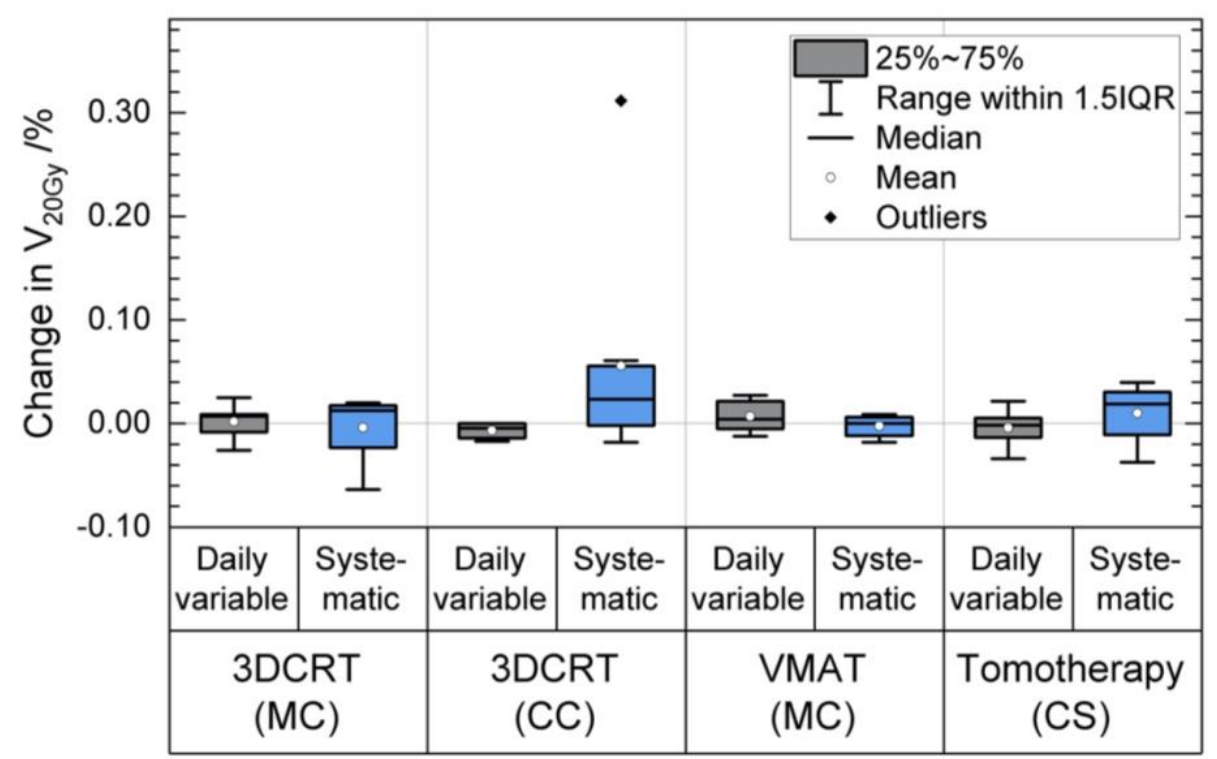

Figure 3.11: The change in $\mathrm{V}_{20 \mathrm{~Gy}}$ of the ipsilateral lung for all patients when internal port errors were modelled in the three treatment techniques (tangential 3DCRT, VMAT and helical Tomotherapy). The modelled daily variable errors are in gray and systematic errors are in blue. The dose calculation engine used with each treatment technique is shown in brackets - MC: Monte Carlo, CC: Collapsed Cone, CS: Convolution/Superposition.

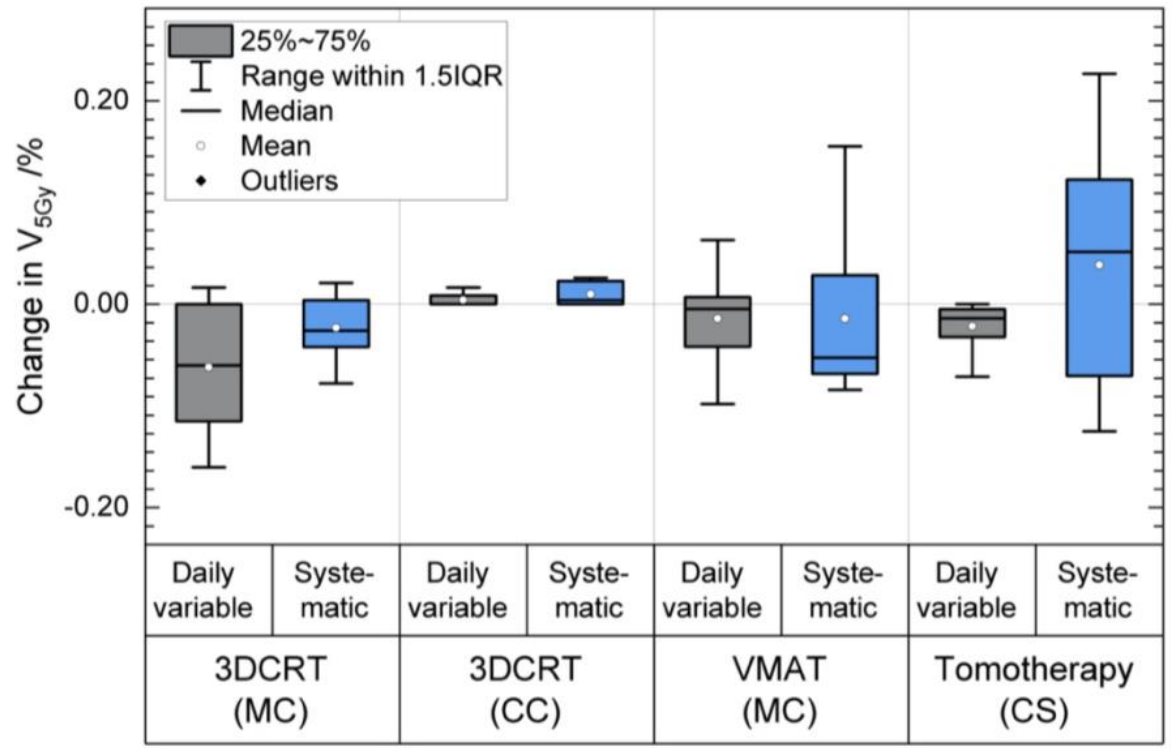

Figure 3.12: The change in $\mathrm{V}_{5 \mathrm{~Gy}}$ of the heart for all patients when internal port errors were modelled in the three treatment techniques (tangential 3DCRT, VMAT and helical Tomotherapy). The modelled daily variable errors are in gray and systematic errors are in blue. The dose calculation engine used with each treatment technique is shown in brackets - MC: Monte Carlo, CC: Collapsed Cone, CS: Convolution/Superposition. 
The cumulative effect of the daily variable and systematic internal port errors resulted in small changes to $\mathrm{V}_{20 \mathrm{~Gy}}$ of the ipsilateral lung and $\mathrm{V}_{5 \mathrm{~Gy}}$ of the heart, all with absolute means of less than $0.5 \%$.

Despite the overall small effect of internal port errors on the relevant OARs, the heart was affected to a larger degree by internal port errors in VMAT and helical Tomotherapy treatment when compared with tangential 3DCRT delivery. In VMAT and Tomotherapy, the radiation is delivered from a wider range of angles around the patient, increasing the dose received by the heart, while in the 3DCRT plans the opposed tangential beams are angled to minimize the exposure to the heart. Therefore, small changes in the position of the metal port can perturb the fields that pass through the heart to a greater extent.

The treatment planning $\mathrm{kVCT}$ contains heavy metal artefacts which introduce an uncertainty when locating and contouring the metal port during treatment planning. This can result in a systematic error in the true position of the metal port. However, the results presented in this chapter indicate that the cumulative dosimetric effect of a systematic internal port error on target coverage and OARs is very small in all treatment techniques.

\subsection{Evaluating density override as a planning strategy}

A potential planning strategy to make treatment delivery more robust against potential port movement is to override the density of the entire tissue expander (including the metal port) with average breast tissue density. This strategy may also remove the uncertainty associated with contouring the metal port during treatment planning, as the presence of the metal artefacts in the TPCT introduces a vagueness in accurately locating the metal port. 
In addition, this method may increase the efficiency of treatment planners, as contouring is a time-consuming step in treatment planning.

The density override of the metal port was performed outside of the TPS, using the same methods described in section 3.2. For each patient, the contour coordinates of the implant were used to identify the voxels that are part of the tissue expander, including the metal port. These voxels were assigned the mean HU value of nearby breast tissue that was free from artefacts. The density override corrected for the metal artefacts and assigned the whole breast a uniform tissue density. It is important to note that density overrides can be implemented directly in the TPS (Monaco and Tomotherapy) during planning, however, in this study, all density corrections were done outside the two TPSs to maintain consistent CT datasets.

Similar to the steps described in section 3.3, the corrected CT data set of each patient was imported back into the DQA feature of the TPS (Monaco and Tomotherapy) as a phantom. The 3DCRT and VMAT plans were recalculated on the imported CT dataset in Monaco, and the helical Tomotherapy plan was recalculated on the imported CT datasets within the DQA station of Tomotherapy. The resulted dose distribution of each calculation was exported in a DICOM format and analyzed outside the TPS. Each dose distribution was then multiplied by the number of delivered fractions to represent a full course of treatment. This was repeated for all patients by comparing the resulting dose distribution against the cumulative dose distribution that accurately modeled the measured daily positional errors. 
The boxplot in Figure 3.13 summarizes the change in $\mathrm{V}_{100 \% \mathrm{Rx}}$ of the ROI when a tissue override was applied to the metal port, relative to the reference dose distribution in all patients for the three treatment techniques.

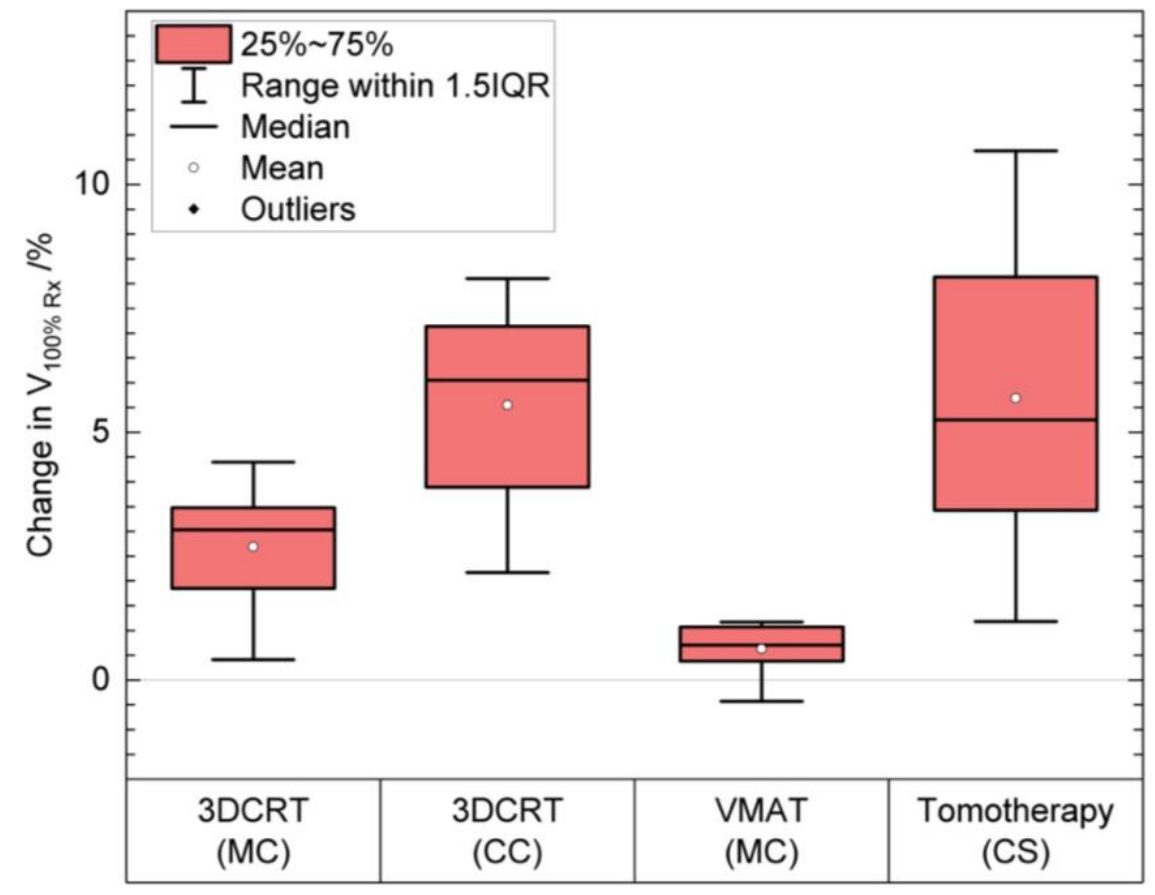

Figure 3.13: The change in $\mathrm{V}_{100 \% \mathrm{Rx}}$ of the ROI for all patients when the metal port was overridden with tissue-equivalent density, relative to the reference dose in the three treatment techniques (tangential 3DCRT, VMAT and helical Tomotherapy). The dose calculation engine used with each treatment technique is shown in brackets - MC: Monte Carlo, CC: Collapsed Cone, CS: Convolution/Superposition.

The mean change in $\mathrm{V}_{100 \% \mathrm{Rx}}$ was $2.7 \%$ (range: $0.4 \%$ to $4.4 \%$ ) for 3DCRT (MC), $6 \%$ (range: $2.2 \%$ to $8.1 \%$ ) for $3 \mathrm{DCRT}(\mathrm{CC}),<1 \%$ (range: $-0.4 \%$ to $1.2 \%$ ) for VMAT (MC) and $6 \%$ (range: $1.2 \%$ to $11 \%$ ) for Tomotherapy (CS). This indicates that if the metal port is not accounted for during treatment planning, the dose delivered to the ROI is overestimated which can lead to an underdosage of the target. This is in agreement with previous studies. ${ }^{45,50,55}$ 
The difference between VMAT and helical Tomotherapy treatments can be attributed to two reasons. First, the beam delivery configuration in the two techniques is different. For all the VMAT plans, the arc was limited to $230^{\circ}$, while helical Tomotherapy uses a continuously-rotating fan beam, potentially irradiating the target from a wider range of directions. Therefore, ignoring the high-density metal port in a helical Tomotherapy plan may expose a larger volume of the ROI to the beam, thus, overestimating $\mathrm{V}_{100 \% \mathrm{Rx}}$ to a greater extent. Second, the dose calculation algorithms used for the two techniques were different. While Tomotherapy uses CS, MC was used to calculate the VMAT dose distributions which models the presence of the metal port differently. Similarly, the difference between the two tangential 3DCRT calculations is attributed to the different modeling of the metal port in MC and CC. MC-based dose calculation engines have been established as being more accurate than $\mathrm{CC}$. Compared with the MC calculation, the $\mathrm{CC}$ calculation overestimated the dose delivered to the target. Therefore, MC should be used when high-density inhomogeneities are present in the field of irradiation.

A clinical implication of the results in Figure 3.13 is that contouring the metal port (despite the contouring uncertainties involved), and assigning it a proper density is an overall better strategy than overriding it with tissue-equivalent density. 


\subsection{Summary}

The dosimetric effect of internal port error was evaluated for eight breast cases when treated with tangential 3DCRT, VMAT and helical Tomotherapy. The cumulative effect of daily variable and systematic internal port errors were evaluated. Results showed that systematic internal port errors had a larger effect on target coverage than daily variable errors, while no discernable effects on OARs were present in all three techniques. For larger and systematic errors, VMAT and helical Tomotherapy were more robust against internal port errors than a tangential 3DCRT configuration.

In addition, a planning strategy of overriding the metal port with tissue-equivalent density for more robust delivery was evaluated in all three techniques. Results showed that ignoring the metal port during treatment planning can overestimate the dose to the target in the TPS, and lead to an underdosage. Therefore, it is recommended to assign the metal port its appropriate densities despite the potential contouring uncertainties that result from the metal artefact. 


\section{Chapter 4: Robustness against patient registration errors}

As introduced in the previous chapter, the measured positional errors of the metal port can be categorized into two classes of error. In Chapter 3, the first class of error referred to as internal port error was modelled and its dosimetric effect in three treatment techniques (3DCRT, VMAT and helical Tomotherapy) was evaluated. In this chapter, the second class of port positional errors will be discussed.

The second class of error is an error in the position of the metal port that resulted from the displacement of the whole patient relative to the treatment beam, referred to herein as patient registration error. Patient registration errors can be caused by necessary compromises made during patient registration. These compromises are clinical judgements that are made every day when aligning the patient on the treatment couch before the daily fraction.

\subsection{Modelling patient registration errors}

To simulate patient registration errors, the whole patient must be shifted relative to the treatment beam by the measured positional errors. First, the metal artefacts in the TPCT of each patient were corrected. This correction was performed using the in-house developed software, following similar steps described in section 3.2. On slices where metal artefacts were present, the $\mathrm{HU}$ values of the implant were modified to an average $\mathrm{HU}$ value of breast tissue in slices free from the metal artefacts. In addition, the components of the metal port were also corrected for, by assigning the magnetic core and the titanium shell their corresponding nominal HU values, as described in more detail in section 3.2. One 
difference from the process described in section 3.2 is that the position of the metal port was not altered. An example of a representative CT slice is shown in Figure 3.3a before the density corrections, and in Figure 3.3b after the corrections. Similar corrections were applied to the TPCT of all breast cases and will be referred to as the corrected $C T$ dataset.

\subsection{Dose calculations and data analysis}

The DQA stations of Monaco and Tomotherapy were used to calculate the treatment plans. First, the corrected CT dataset of each patient was imported as DQA phantoms to the TPS. In each TPS, patient registration errors were simulated by shifting the corrected CT dataset of each patient relative to the planned photon fluence and by recalculating the original plan. The shifting of the CT dataset relative to the photon fluence was achieved differently in each TPS.

In Monaco, before every DQA calculation, the system prompts the user to input the three-dimensional coordinates of the isocenter of the plan. Therefore, to replicate the same port positional errors that resulted from the displacement of the whole patient relative to the treatment beam, the isocenter of the treatment must be shifted by the same magnitude as the measured error but in the opposite direction. For each fraction, the isocenter of the plan was adjusted to match the measured error of that treatment fraction, and the original plan (3DCRT and VMAT) was recalculated. The resulted dose distribution of each simulated fraction was then exported from Monaco in a DICOM format for further analysis.

In the DQA station of Tomotherapy, the original isocenter of the plan applies automatically to the imported CT dataset of the patient. The Phantom Tools allow to 
reposition the imported phantom (i.e., the corrected CT dataset) with respect to the planned photon fluence. The repositioning is done manually by dragging the phantom with the mouse in the three cardinal directions, as opposed to inputting a specified coordinate of the isocenter. The magnitude of the shift in each direction was verified by displaying the imaging borders of the phantom and the fluence maps and measuring the distance of the borders using the coordinate displaying function. For each fraction, the corrected CT dataset was repositioned by the measured error in that fraction, and the original plan was recalculated. The resulted dose distributions were then exported in a DICOM format for further analysis.

Regardless of the dose calculation algorithm, Monaco bounds its dose calculation inside the patient only (i.e., it does not calculate dose outside the patient), which is dictated by the "external" contour defined by the planner. For that reason, the size of the exported dose distribution matrix was inconsistent from patient to patient for a given dose calculation grid size. Moreover, for a given patient, the dimensions of the exported dose distributions of different fractions varied by one row/column with no apparent pattern, while the number of slices remained constant. This has posed a challenge when various matrix operations were performed on the dose distributions outside of the TPS, as there was no one-to-one correspondence between voxels. To solve this problem, the DICOM tag "Image Position (Patient) Attribute" was used. This tag specifies the three-dimensional coordinate of the upper left-hand corner (center of voxel) of the dose distribution matrix. Using this information, along with the voxel dimensions, the spatial coordinates of all the voxels in a dose distribution were determined. In turn, this allowed matching and overlaying dose distribution matrices of different modelled fractions. 
In Tomotherapy the dose calculation grid is not limited to the external bounds of the patient and has a constant width and height of $600 \mathrm{~mm}$ for all modelled fractions. The only variable that is different among patient is the depth, which depends on the number of slices acquired in the TPCT. Therefore, there was a one-to-one voxel correspondence between different modelled fractions of the same patient.

The evaluation of the dosimetric effect of patient registration errors in the different treatment techniques was done in a similar manner as described in sections 3.3 and 3.4. The percent dose differences between the investigated scenarios and the reference dose distribution were calculated. The reference dose for each technique was the original plan calculated on a corrected CT dataset without any shifts applied. The dosimetric evaluation in biologically relevant areas of the target was done by analyzing the ROI that was previously defined in section 3.3 as a $5 \mathrm{~mm}$ expansion around the temporary implant within the PTV. This ROI contains tissue that is vulnerable to being affected by the presence of the metal port and its positional errors. Relative changes in clinically relevant DVH metrics were calculated for the ROI and OARs such as the ipsilateral lung and heart.

\subsection{Results: dosimetric effect of daily measured errors and a systematic error}

For all eight breast cases, the dosimetric effects of patient registration errors in each of the three treatment techniques (tangential 3DCRT, VMAT and helical Tomotherapy) were evaluated for two scenarios. The first scenario was the cumulative effect of the daily measured error, also referred to as "daily variable" error. The second scenario was the cumulative effect of a systematic error, also referred to as "systematic" error. 
For the first modelled scenario, the cumulative effect of daily measured patient registration errors was evaluated. The magnitude and direction of the modelled registration errors for each patient were identical to the measured inter-fractional positional error of the metal port presented in Chapter 2. The plan of each technique was recalculated on the corrected $\mathrm{CT}$ dataset, while displacing the $\mathrm{CT}$ with respect to the plan by the magnitude of the measured error of every fraction. The resulting 25 new dose distributions, each representing a treatment fraction, were summed for a cumulative dose distribution and compared against the original planned dose distribution. Figure 3.4 shows for a representative patient the original planned dose distribution in each of the three techniques. Figure 4.1 shows for the same representative patient the percent dose difference map when daily variable patient registration errors were modelled in the three techniques. The 3DCRT plans were calculated using both MC and CC dose calculation algorithms. 


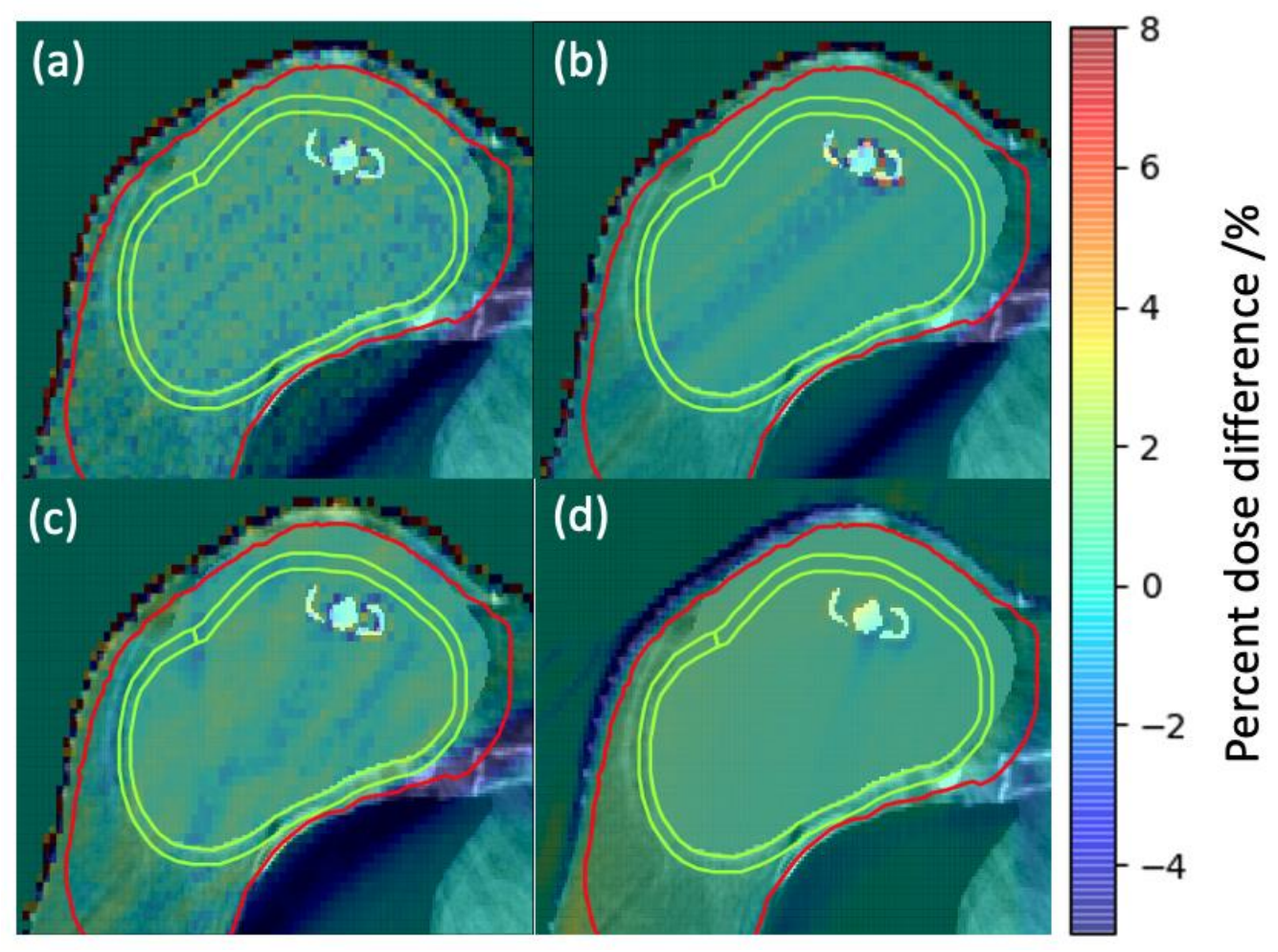

Figure 4.1: Percent dose difference maps for a representative slice and patient when daily variable patient registration errors were modelled in 3DCRT MC (a), 3DCRT CC (b), VMAT MC (c) and helical Tomotherapy CS (d). All percent dose differences were normalized to Rx. The red contour is the PTV and the area bound by the yellow contour is the ROI (defined as a $5 \mathrm{~mm}$ expansion around the saline implant).

For the second modelled scenario, the cumulative effect of a systematic patient registration error was evaluated. This represents a scenario where a change to the patient anatomy occurred between the simulation scan and the first treatment fraction which led to a positioning offset of the patient that persisted throughout the course of the treatment. The magnitude and direction of the error was identical to the systematic error modelled in section 3.4, which was derived from the largest positional error measured during metal port registration. The plan of each of the three techniques was recalculated on the corrected CT dataset while the whole $\mathrm{CT}$ was shifted by the specified magnitude relative to the isocenter 
of the plan. The resulted dose distribution was then multiplied by the number of treatment fractions to represent a full course of treatment, and compared against the originally planned dose. Figure 4.2 shows for the same representative patient the percent dose difference maps from the reference dose when a systematic patient registration error was modelled in the three treatment techniques.

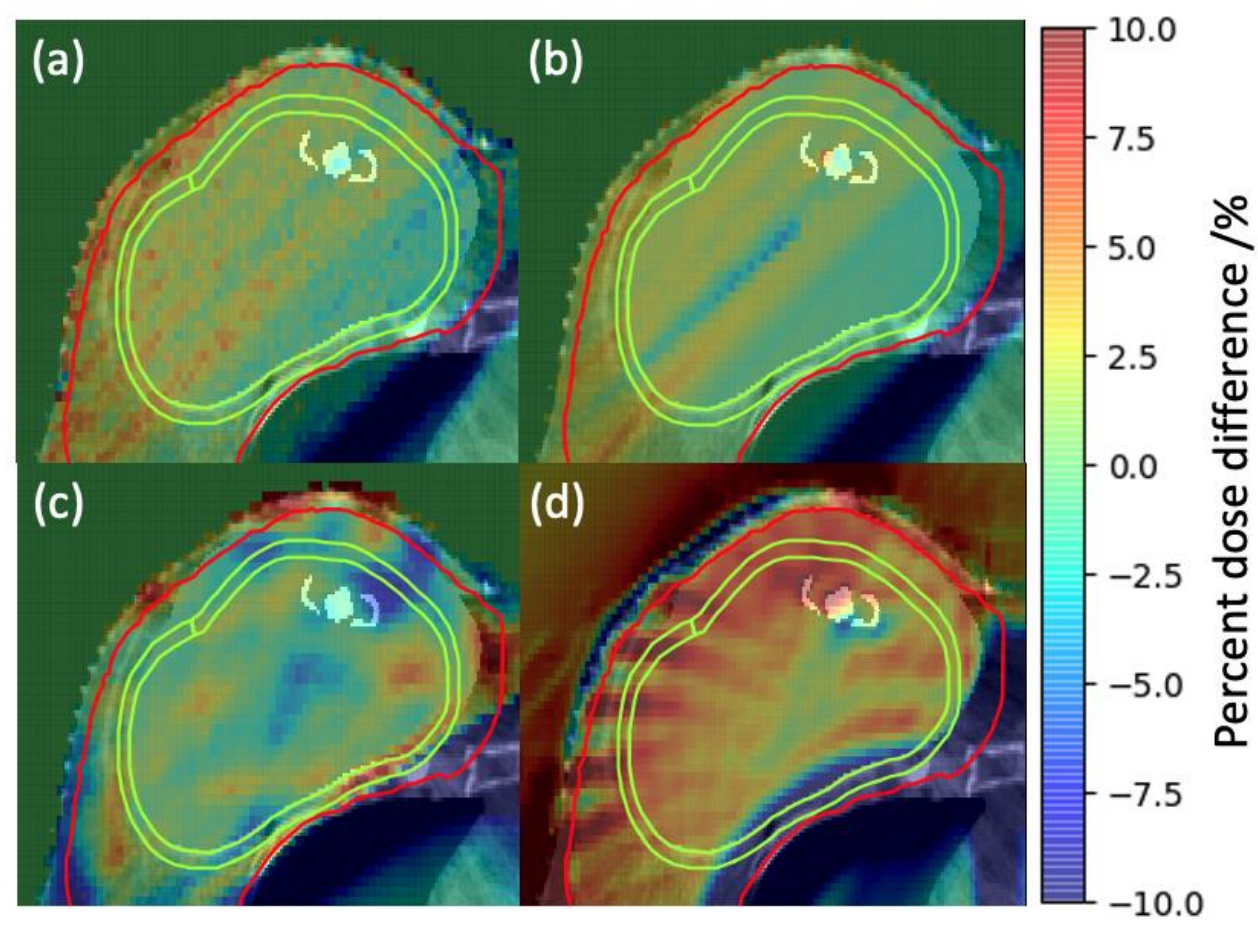

Figure 4.2: Percent dose difference maps for a representative slice and patient when a systematic patient registration error was modelled in 3DCRT MC (a), 3DCRT CC (b), VMAT MC (c) and helical Tomotherapy CS (d). All percent dose differences were normalized to Rx.

When a systematic registration error was modelled, more extensive perturbations to the dose distributions were present in all three techniques, compared to the effect of daily variable registration errors. Larger areas of the breast and the ipsilateral lung were affected by the positioning error, which extended beyond the temporary implant. 
From an analysis of all patients, the percent point dose differences inside the ROI when daily variable and systematic patient registration errors were modelled in the three treatment techniques are summarized in in the boxplot in Figure 4.3.

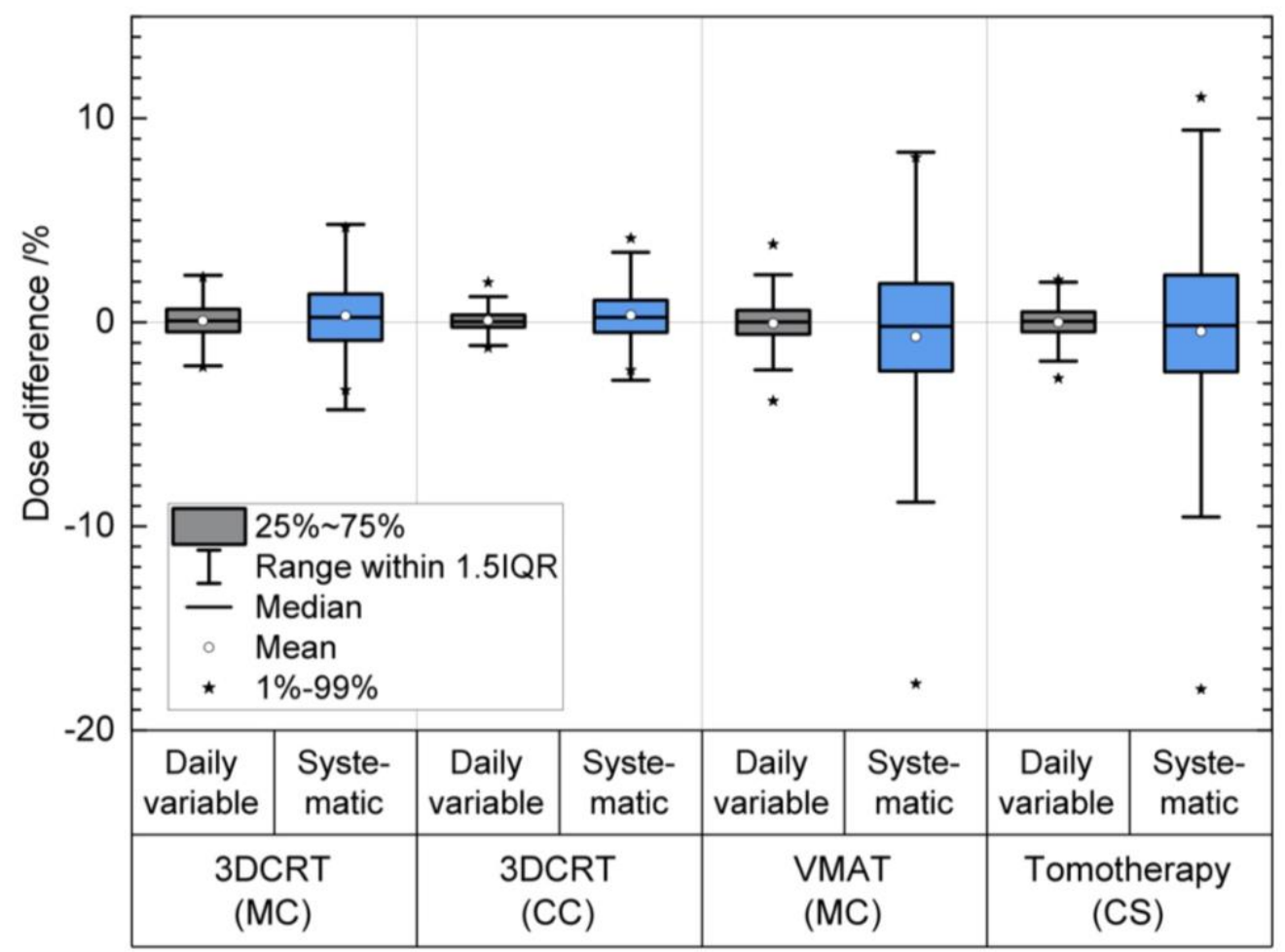

Figure 4.3: Percent point dose differences inside the ROI for all patients when patient registration errors were modelled in the three treatment techniques (tangential 3DCRT, VMAT and helical Tomotherapy). The modelled daily variable errors are in gray and systematic errors are in blue. All point dose differences were normalized to Rx. The dose calculation engine used with each treatment technique is shown in brackets MC: Monte Carlo, CC: Collapsed Cone, CS: Convolution/Superposition.

The cumulative effect of the daily variable and systematic patient registration errors resulted in an absolute mean point dose differences of $<0.7 \%$ in the three treatment techniques. For daily variable errors, the interquartile range of point dose differences was 
small, and within $1 \%$ in all three techniques. For a systematic error, the interquartile range was larger, and within $2.5 \%$ in all three techniques.

The $1 \%-99 \%$ range for daily variable registration errors were within $2.5 \%$ for 3DCRT (MC), $2 \%$ for 3DCRT (CC), 4\% for VMAT (MC) and 2\% for Tomotherapy (CS) treatment plans. For a systematic registration error, the $1 \%-99 \%$ range in point dose differences were within $4.5 \%$ for 3DCRT (MC), $4 \%$ for 3DCRT (CC), $-18 \%$ to $8 \%$ for VMAT (MC) and $-18 \%$ to $11 \%$ for Tomotherapy (CS).

The VMAT and helical Tomotherapy plans resulted in higher point dose variability in the target when compared with the tangential 3DCRT plan. VMAT and helical Tomotherapy treatments are delivered with small collimated beamlets that conform to the target volume, as opposed to the open fields that are used with 3DCRT tangents. Therefore, small shifts in the position of the patient relative to those small beamlets can alter the dose delivered to the target volume. The high variability in point dose differences in VMAT and helical Tomotherapy treatments did not result in a distinct area of underdosage, and depended on the direction of the modelled registration error.

The DVH of the ROI is shown in Figure 4.4 for a representative patient. The original DVH (when no error was modelled) is compared against the modelled scenarios of daily variable and systematic patient registration errors in each of the three techniques. Similar DVH curves were generated for all patients, and the change in $\mathrm{V}_{100 \% \mathrm{Rx}}$ (volume of ROI receiving Rx) was calculated when daily variable and systematic patient registration errors were modelled in each of the three techniques. The box plot in Figure 4.5 summarizes the change in $\mathrm{V}_{100 \% \mathrm{Rx}}$ of the ROI for all patients. 


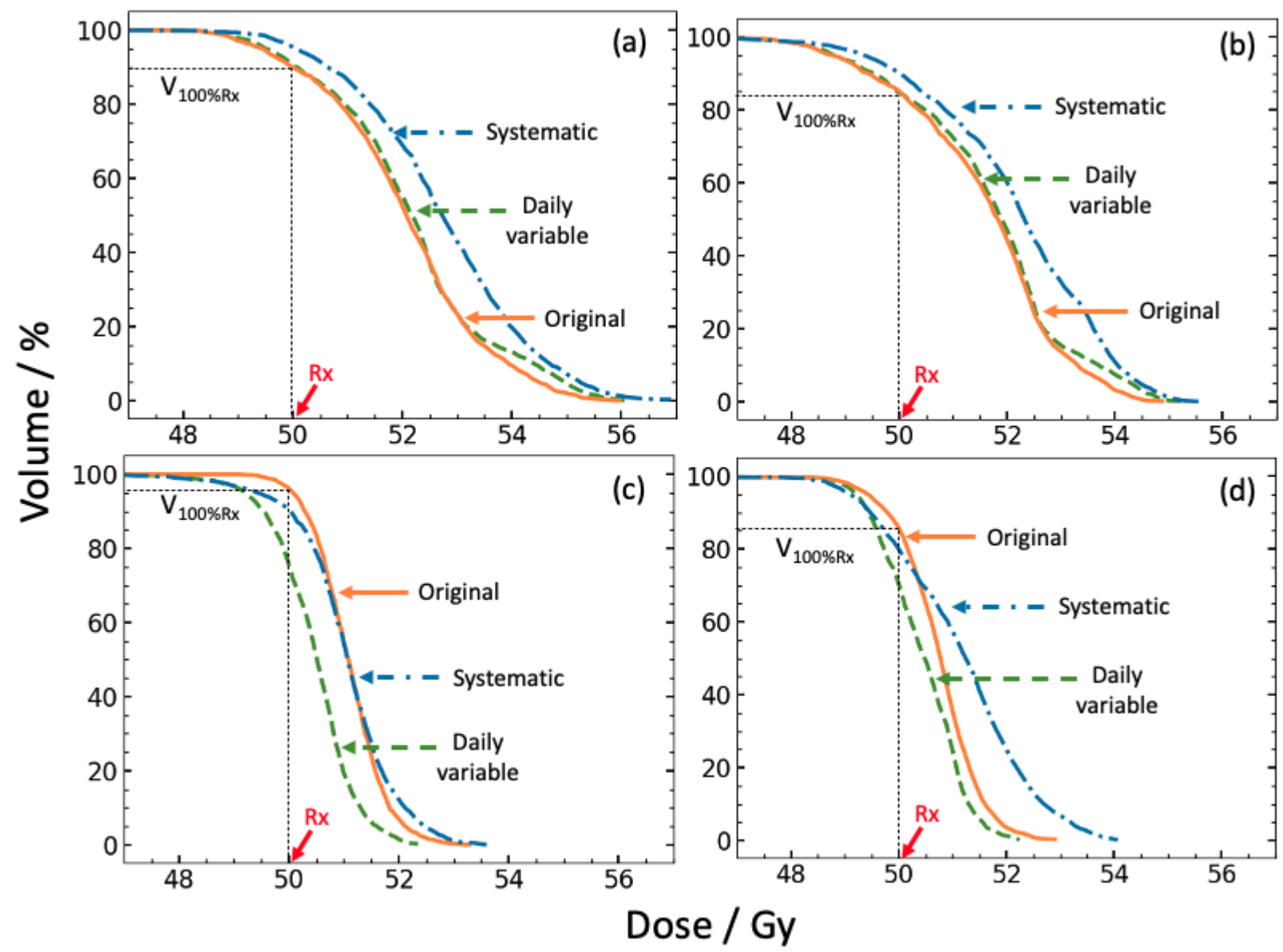

Figure 4.4: DVH of the ROI for a representative patient when patient registration errors were modelled in 3DCRT MC (a), 3DCRT CC (b), VMAT MC (c) and helical Tomotherapy CS (d). Solid orange: original plan. Green dashed: cumulative dose with daily variable port error modelled. Blue dash-dotted: cumulative dose with a systematic port displacement modelled. 


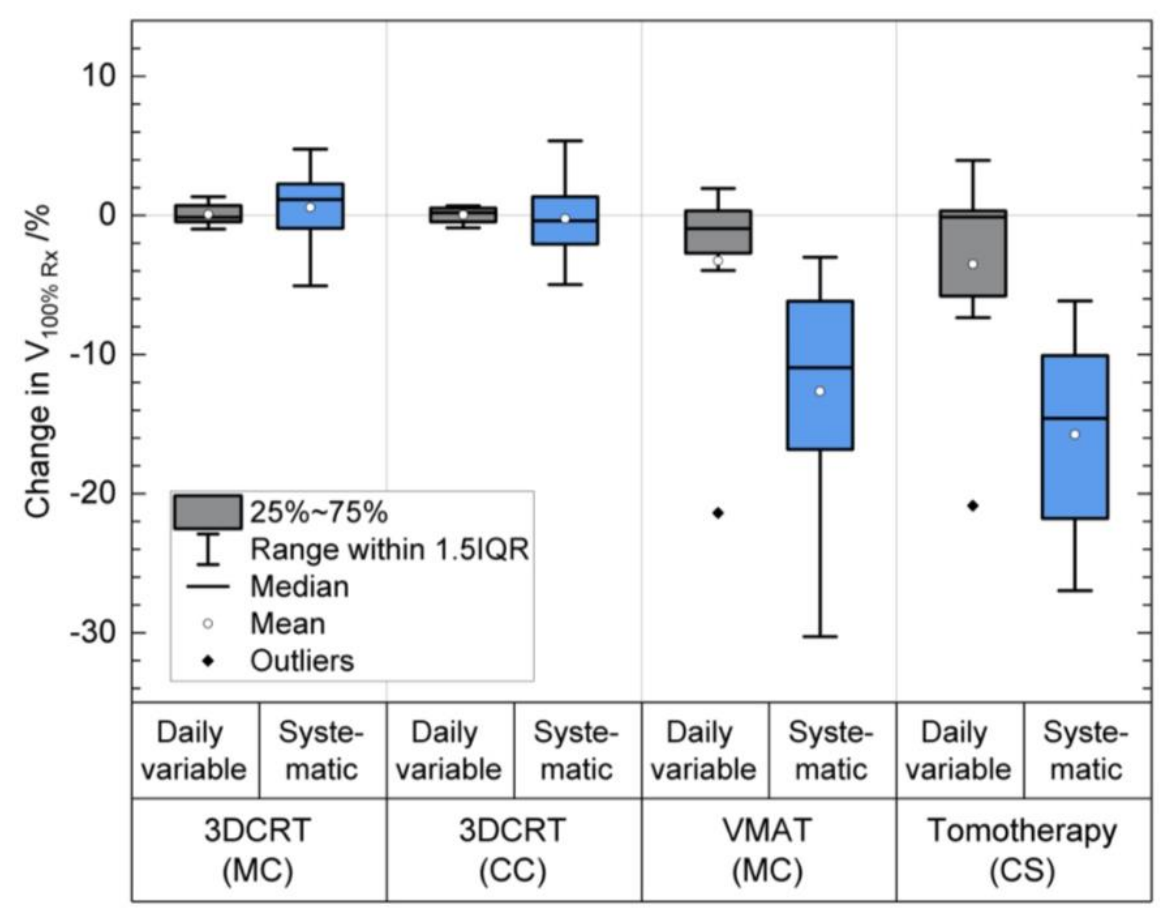

Figure 4.5: The change in $\mathrm{V}_{100 \% \mathrm{Rx}}$ of the $\mathrm{ROI}$ for all patients when patient registration errors were modelled in the three treatment techniques (tangential 3DCRT, VMAT and helical Tomotherapy). The modelled daily variable errors are in gray and systematic errors are in blue. The dose calculation engine used with each treatment technique is shown in brackets - MC: Monte Carlo, CC: Collapsed Cone, CS: Convolution/Superposition.

The cumulative effect of the daily variable patient registration errors resulted in a mean change of $<0.1 \%$ (range: $-1 \%$ to $1.3 \%$ ) for 3 DCRT (MC), $<0.1 \%$ (range: $-0.9 \%$ to $0.7 \%$ ) for 3DCRT (CC), $-3 \%$ (range: $-21 \%$ to $1.9 \%$ ) for VMAT (MC) and $-3.5 \%$ (range: $-21 \%$ to $4.0 \%$ ) for Tomotherapy (CS). The cumulative effect of a systematic patient registration error resulted in a mean change of $-0.2 \%$ (range: $-5.1 \%$ to $4.8 \%$ ) for 3DCRT (MC), $0.6 \%$ (range: $-5.0 \%$ to $5.4 \%$ ) for $3 \mathrm{DCRT}(\mathrm{CC}),-13 \%$ (range: $-30 \%$ to $-3.0 \%$ ) for VMAT (MC) and -16\% (range: $-27 \%$ to $-6.1 \%$ ) for Tomotherapy (CS). 
The change in $\mathrm{V}_{100 \% \mathrm{Rx}}$ of the ROI shows outliers when the daily variable patient registration errors were modelled in VMAT and helical Tomotherapy treatments (Figure 4.5). These outliers are not present in the tangential 3DCRT treatment. The origin of these outliers is from one patient who received a bilateral reconstruction and treatment. For that patient, the daily measured positional errors were systematically offset for both breasts in the three cardinal directions, as seen in Figure 2.4, indicated by patient indices 3 and 4. In a tangential 3DCRT treatment, the target coverage is less affected by daily variable and systematic patient positioning errors, as the two main tangential fields are open anteriorly, and the target remains within the radiation field with relatively small patient displacements. In a VMAT or a helical Tomotherapy treatment, the delivered dose is more conformal, therefore, the target coverage is compromised to a greater extent when patient registration errors are present.

The relatively small effect that patient registration errors have on target coverage in the tangential 3DCRT treatments can also be explained by the unique shape of the ROI examined in this study. The studied cohort of patients was originally treated on Tomotherapy. In our institution, in addition to bilateral treatments, patients with temporary tissue expanders who have a large chest wall curvature are treated on Tomotherapy (see Figure 2.1), as it can deliver a conformal dose to a complex anatomy. Therefore, the shape of the PTV for some of these patients is more concave in the posterior end, making the ROI a $5 \mathrm{~mm}$ loop that curves inwards by the curvature of the chest wall. As the position of the patient shifts relative to the tangential beams, and depending on the direction of the error, only a small volume of the ROI (the vertices) is displaced out of the field of radiation. For 
these patients, the tangential treatments are more robust against patient positioning errors when the target coverage is assessed.

For the relevant OARs, the DVH of the ipsilateral lung and the heart are shown in Figure 4.6 for a representative patient. The original DVH curves of the ipsilateral lung and the heart are compared against the cumulative effect of daily variable and systematic patient registration errors in each of the three treatment techniques.

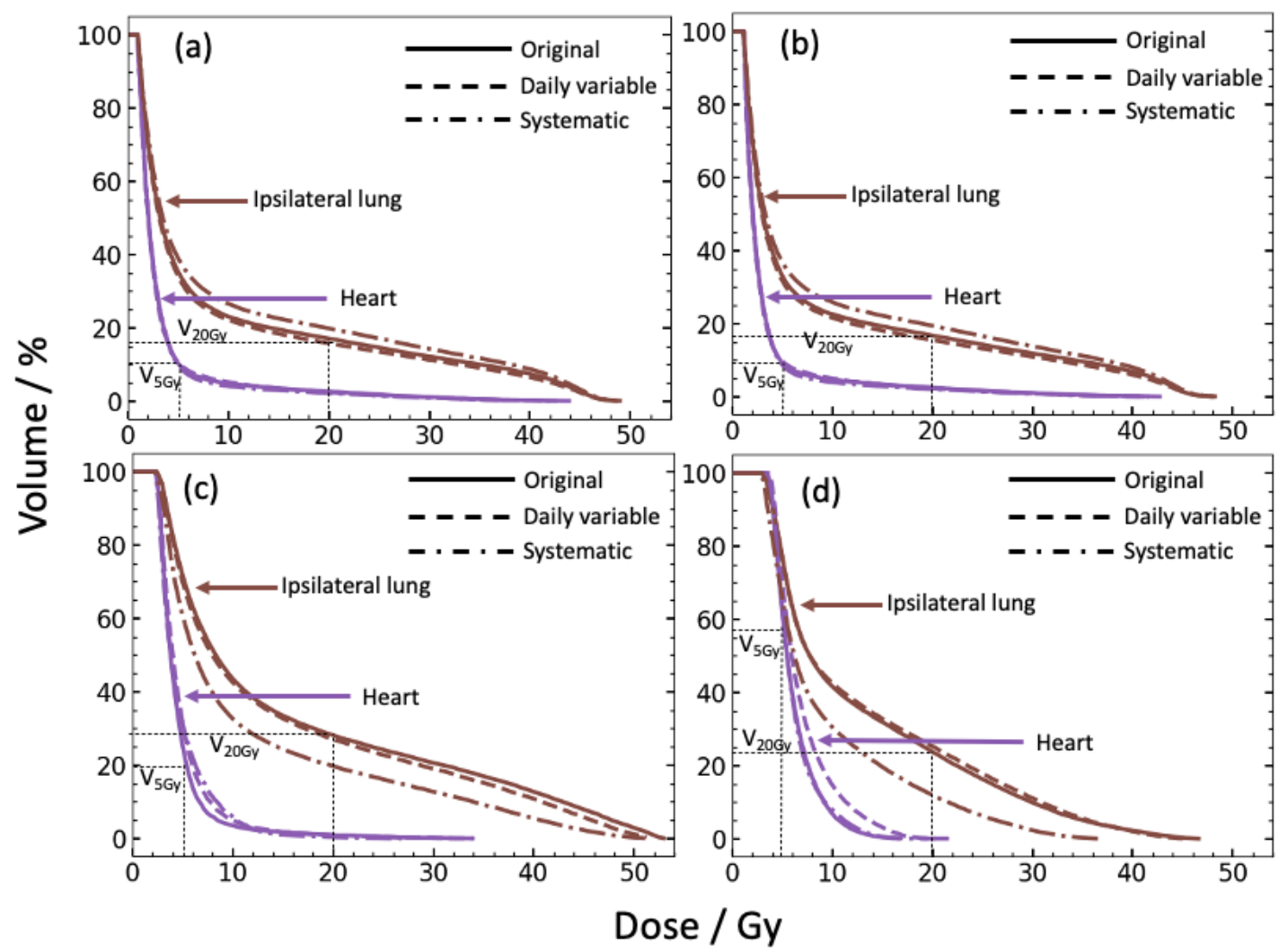

Figure 4.6: DVH of the ipsilateral lung (in brown) and the heart (in purple) for a representative patient when internal port positional errors were modelled in 3DCRT MC (a), 3DCRT CC (b), VMAT MC (c) and helical Tomotherapy CS (d). Solid: original plan. Dashed: daily variable error modelled. Dash-dotted: systematic error modelled. The metrics $\mathrm{V}_{20 \mathrm{~Gy}}$ of the lung and $\mathrm{V}_{5 \mathrm{~Gy}}$ of the heart are shown. 
Similar DVH curves were generated for all patients, and the relative change in $\mathrm{V}_{\text {20Gy }}$ of the lung and $\mathrm{V}_{5 \mathrm{~Gy}}$ of the heart were calculated for the modelled errors per each of the three techniques. Figure 4.7 and Figure 4.8 summarize for all patients the change of these DVH metrics per technique.

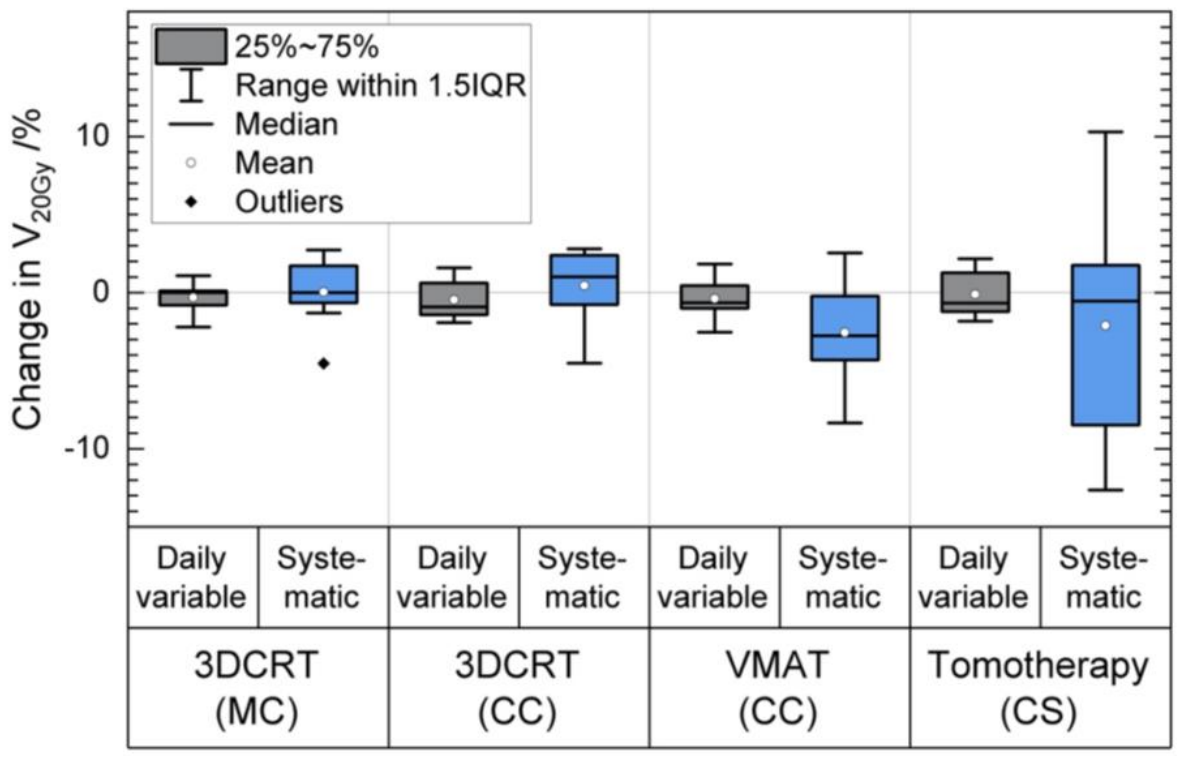

Figure 4.7: The change in $\mathrm{V}_{20 \mathrm{~Gy}}$ of the ipsilateral lung for all patients when patient registration errors were modelled in the three treatment techniques (tangential 3DCRT, VMAT and helical Tomotherapy). The modelled daily variable errors are in gray and systematic errors are in blue. The dose calculation engine used with each treatment technique is shown in brackets - MC: Monte Carlo, CC: Collapsed Cone, CS: Convolution/Superposition. 


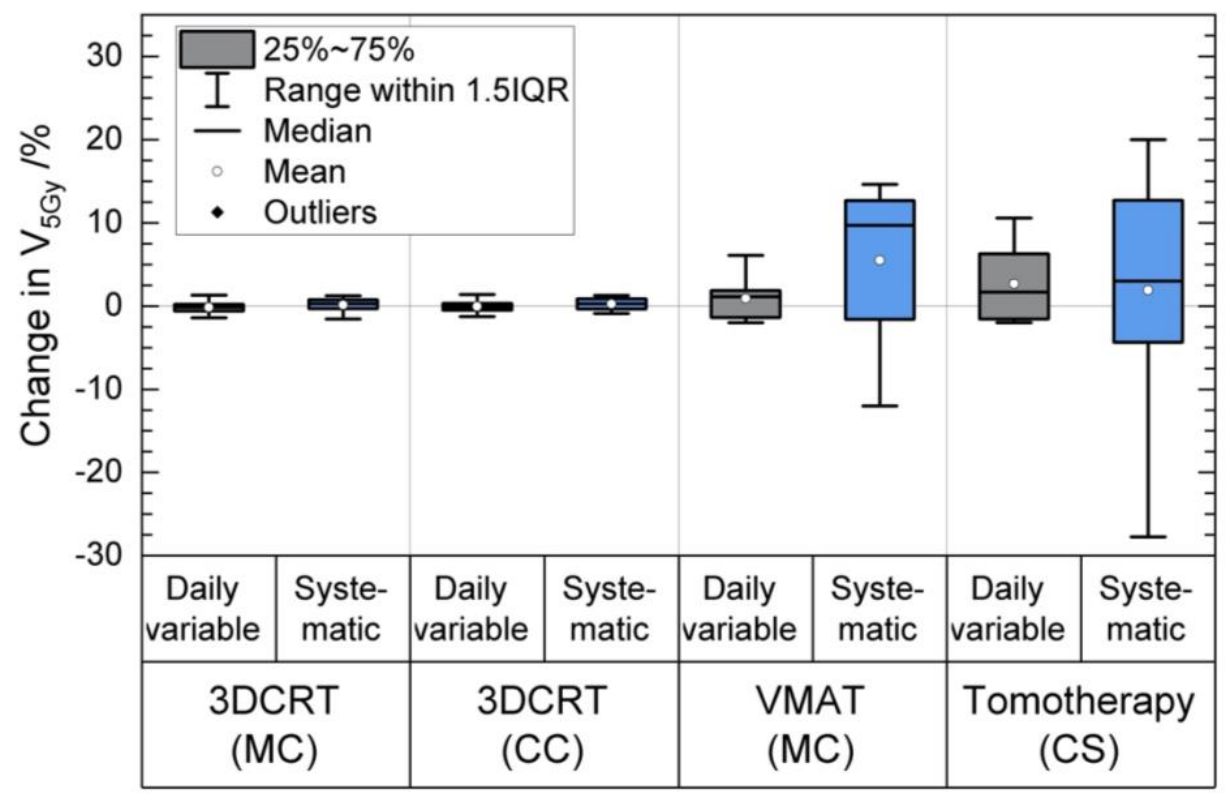

Figure 4.8: The change in $\mathrm{V}_{5 \mathrm{~Gy}}$ of the heart for all patients when patient registration errors were modelled in the three treatment techniques (tangential 3DCRT, VMAT and helical Tomotherapy). The modelled daily variable errors are in gray and systematic errors are in blue. The dose calculation engine used with each treatment technique is shown in brackets - MC: Monte Carlo, CC: Collapsed Cone, CS: Convolution/Superposition.

When daily variable patient registration errors were modelled, changes to $\mathrm{V}_{20 \mathrm{~Gy}}$ for the ipsilateral lung were less than $2 \%$ in all three techniques. Changes to $\mathrm{V}_{5 \mathrm{~Gy}}$ of the heart were less than $0.1 \%$ when daily variable patient registration errors were modelled in tangential 3DCRT (MC and CC) treatments, however, for VMAT (MC) and helical Tomotherapy (CS), $\mathrm{V}_{5 \mathrm{~Gy}}$ had a maximum increase of $6 \%$ and $10 \%$, respectively, in a leftsided breast irradiation. In addition, it should be noted that the originally planned $\mathrm{V}_{5 \mathrm{~Gy}}$ in VMAT and Tomotherapy was much higher than in the tangential 3DCRT plans. While VMAT and helical Tomotherapy offer high target volume conformity, larger volumes of the heart and ipsilateral lung are exposed to the radiation beam, when compared with the tangential configuration of 3DCRT. 
When a systematic patient registration error was modelled, V20Gy of the ipsilateral lung increased by as much as $2.8 \%$ for 3DCRT (MC and CC), $2.6 \%$ for VMAT (MC) and $2.4 \%$ for Tomotherapy (CS). For the heart, changes to $\mathrm{V}_{5 \mathrm{~Gy}}$ were less than $2 \%$ in the tangential 3DCRT treatments (MC and CS); however, for VMAT (MC) and helical Tomotherapy (CS), $\mathrm{V}_{5 \mathrm{~Gy}}$ increased by as much as $14 \%$ and $20 \%$, respectively.

The extent and the magnitude of the effects on the OARs depends on the direction of the displacement of patient relative to the treatment beam. Registration errors in VMAT and helical Tomotherapy treatments resulted in a greater effect on OARs than a tangential 3DCRT treatment. This can be explained by the differences in the delivery configurations of these techniques. Small shifts of the patient relative to the beamlets that compromise VMAT and helical Tomotherapy plans can displace a region of the ipsilateral lung and heart in/out the field of radiation. An additional aspect to consider is that two of the patients investigated in this study received a bilateral treatment on Tomotherapy. Although each breast was examined separately, the Tomotherapy plans were bilateral, therefore, small displacements of these patients can expose parts of the lung and heart to the contralateral field of radiation. 


\subsection{Summary}

The dosimetric effect of patient registration error was evaluated for eight breast cases treated with tangential 3DCRT, VMAT and helical Tomotherapy plans. The cumulative effect of daily variable and systematic patient registration errors were considered. Results showed that patient registration errors had larger effects on target coverage and OARs than internal port errors. When patient registration errors were present, the target coverage and OARs were affected the most in VMAT and helical Tomotherapy treatments. 


\section{Chapter 5: Special case}

A special additional case of a patient treated on Tomotherapy with 25 fractions was investigated separately and is presented in this chapter. The dosimetric effect of the measured inter-fractional positional errors for this patient was retrospectively quantified and compared with tangential 3DCRT and VMAT treatments.

\subsection{Measured inter-fractional port error}

For the special additional patient, the tissue expander was loose within the breast pocket, causing the implant to flip from fraction to fraction, with some fractions the metal port being very close to the chest wall, and with other fractions the metal port being very close to the anterior of the breast. Biocell tissue expanders are macrotextured to induce tissue adhesion which keeps the implant immobile and in place. ${ }^{62}$ Improper adhesion of the tissue around the tissue expander, or the use of a smooth expander are plausible explanations to mobility of the implant within the breast pocket. Figure 5.1 shows an MVCT of two treatment fractions fused with the TPCT where the position of the metal port matched and completely did not match its position in the TPCT. 


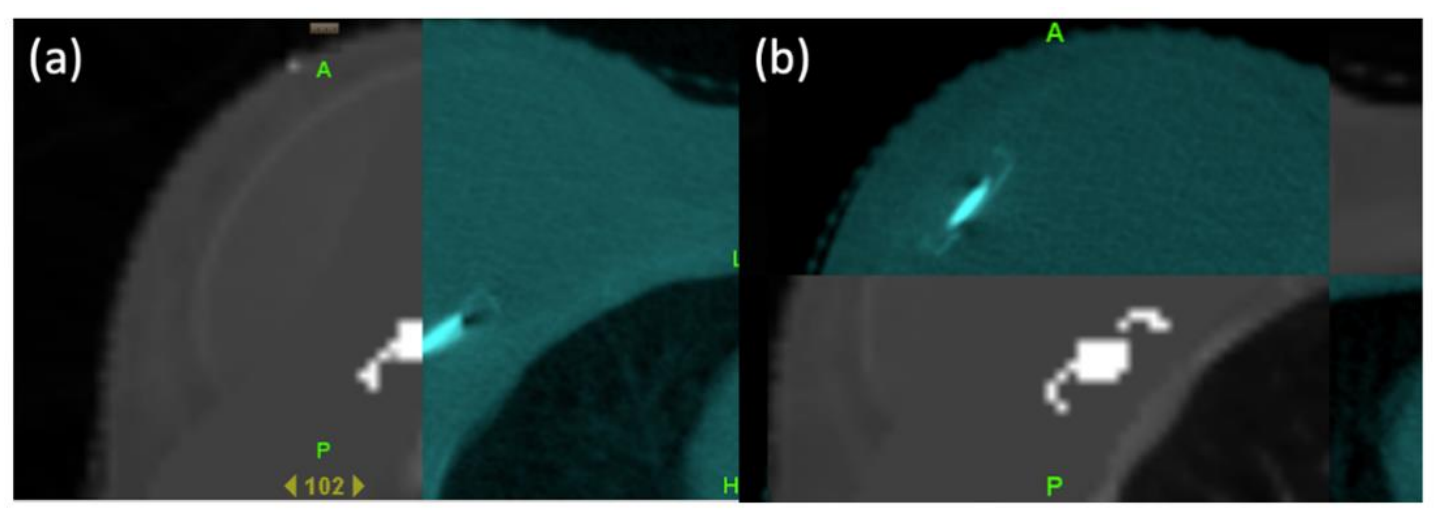

Figure 5.1: Checkered view of an MVCT image of two different fractions in the treatment position (in turquoise) superimposed on the TPCT (in gray) showing a good match in the position of the metal port in one fraction (a), and a large internal displacements of the metal port for a another fraction (b).

The inter-fractional positional variations of the metal port were measured following the same methods described in section 2.2, by re-adjusting the daily registered MVCT and TPCT until the metal port is aligned in both scans in all directions. It should be noted that for this special case, the whole tissue expander was inverted, resulting not only in a large displacement of the metal port, but a change in its orientation, such that it "faced" the posterior of the breast, as seen in Figure 5.1. A perfect alignment of the metal port in the MVCT and TPCT would require using a roll correction in addition to the shifts in the three cardinal directions. However, to avoid complex modelling of the movement of the metal port, shifts in the three cardinal directions only were applied. The alignment of the metal port in the MVCT and TPCT was therefore performed by aligning the core of the metal port, while ignoring the titanium shell. Figure 5.2, shows the distribution of the metal port in a box plot formal in the three cardinal directions. 


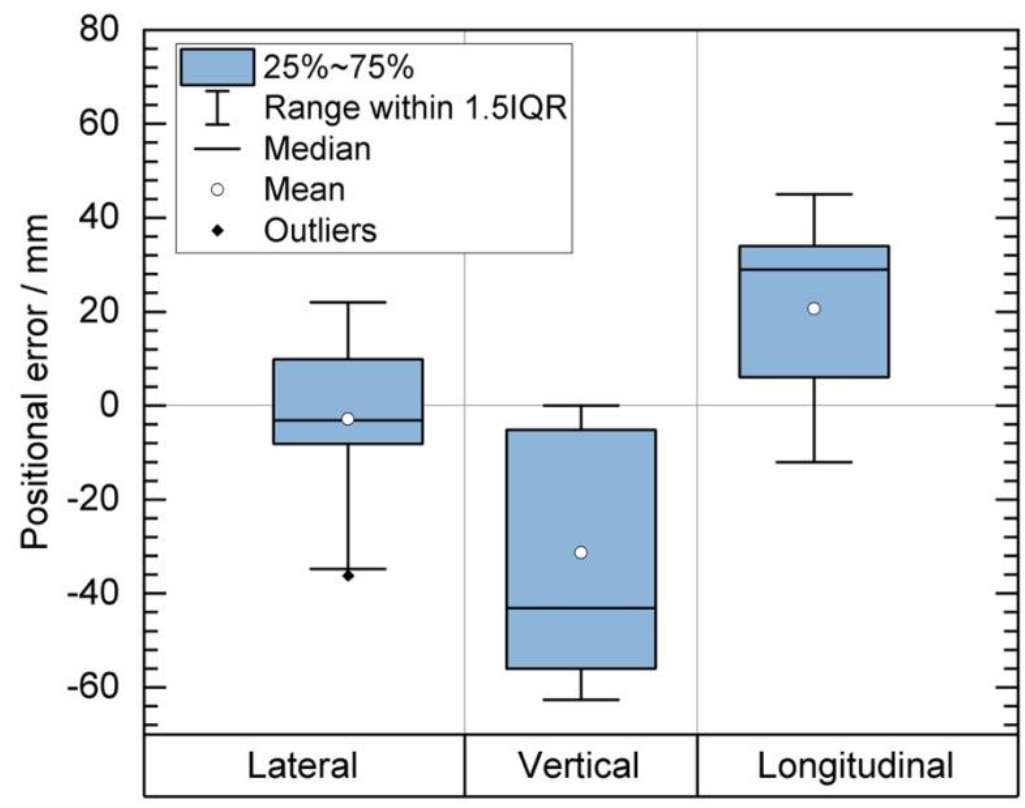

Figure 5.2: Distribution of the measured inter-fractional positional errors of the metal port for the special case in the three cardinal directions. The same sign convention is followed here as described in section 2.3.

The positional error in the lateral direction ranged from $-36 \mathrm{~mm}$ to $22 \mathrm{~mm}$ with a mean error of $-2.9 \mathrm{~mm}$. In the vertical direction, the positional error ranged from $-62 \mathrm{~mm}$ to $0.0 \mathrm{~mm}$ with a mean error of $-31 \mathrm{~mm}$. In the longitudinal direction, the measured error ranged from $-12 \mathrm{~mm}$ to $45 \mathrm{~mm}$ with a mean error of $21 \mathrm{~mm}$. Out of the total 25 fractions, in 11 fractions the metal port was on the same side of the breast as originally in the TPCT (i.e., closer to the chest wall). For the rest 14 fractions, the metal port was on the anterior side of the breast, closer to the skin. The days on which the tissue expander was displaced appeared to be random. 


\subsection{Results: dosimetric effect of daily measured errors}

The robustness of the three techniques (tangential 3DCRT, VMAT and helical Tomotherapy) against inter-fractional positional errors was evaluated for the daily variable internal port errors only. Patient registration errors were not modelled because positioning errors of a magnitude equivalent to the measured errors for this patient are very unlikely to occur. The measured daily error was modelled following the same procedure described in section 3.2. The TPCT of the patient was modified by directly editing voxel values such that the metal port was artificially shifted by the measured magnitude of the error for that fraction, resulting in 25 modified CT datasets. A treatment plan for each of the three techniques was generated, following the same guidelines described in section 3.1. Each treatment plan (3DCRT, VMAT and helical Tomotherapy) was calculated on the 25 modified CT sets and summed for a cumulative dose distribution. The same steps for dose calculation and dose analysis were followed as in section 3.3. The cumulative dose was then compared with the original planned dose distribution of each respective technique. Figure 5.3 shows the percent dose difference maps from the originally planned dose to the cumulative effect of daily measured internal port error. For the tangential 3DCRT plan, both MC and CC dose calculation algorithms were used. 


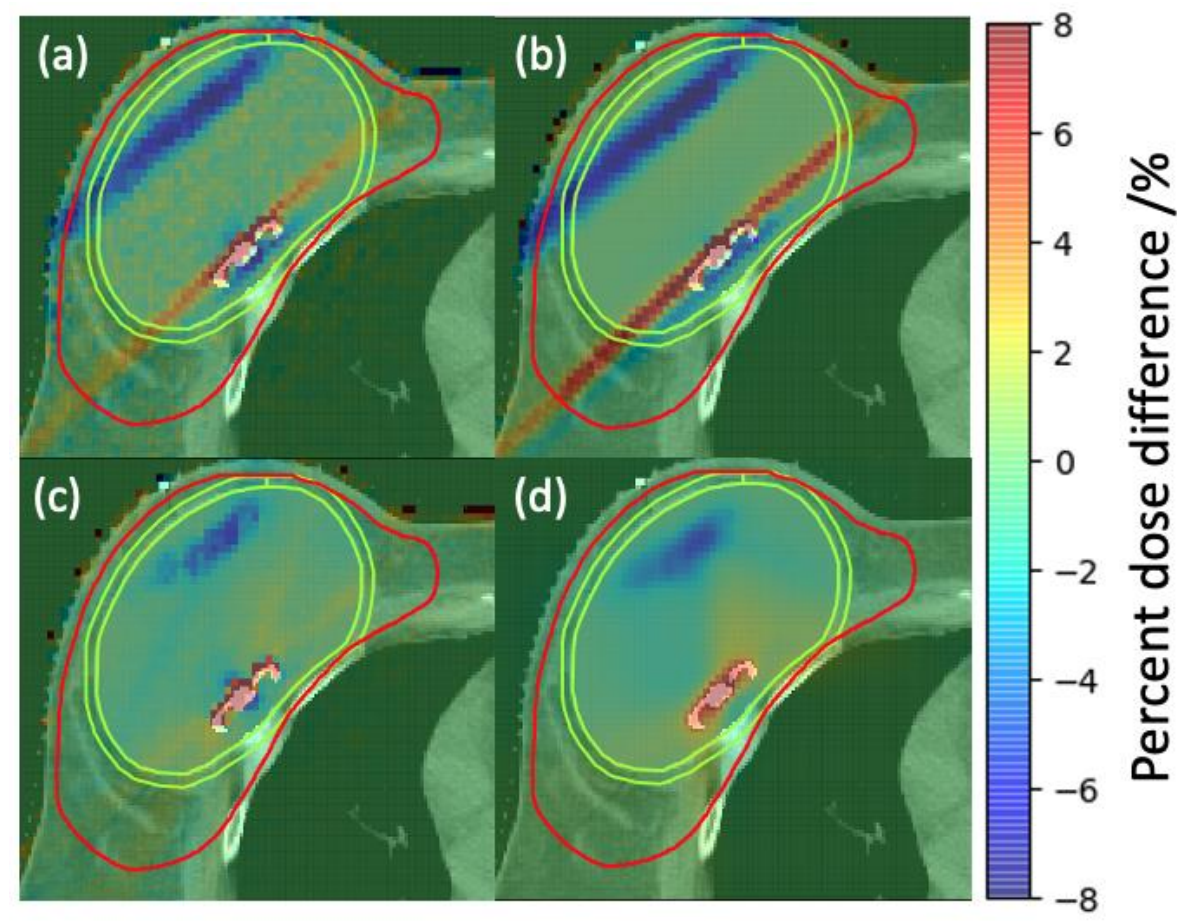

Figure 5.3: Percent dose difference maps for a represntative slice of the special patient when daily variable internal port errors were modelled in 3DCRT MC (a), 3DCRT CC (b), VMAT MC (c) and helical Tomotherapy CS (d). All percent dose differences were normalized to Rx. The red contour is the PTV and the area bound by the yellow contour is the ROI (defined as a $5 \mathrm{~mm}$ expansion around the saline implant).

From Figure 5.3, it is observed that an area of underdosage is present at the anterior side of the breast closer to the skin, and an area of increased dose is observed in the posterior side of the breast closer the chest wall. The underdosage was caused by the presence of the metal port in fractions where the tissue expander was mobilized, and the metal port was shifted to the anterior end of the breast. The increase in dose was caused by the absence of the metal port from its original position in the TPCT. The dose variation in the VMAT and helical Tomotherapy plans was contained within the non-biological implant, and can be viewed as a washout of the effect of the large daily variability in the position of the port. For the 3DCRT plan, an underdosed streak was formed in shadow of 
the metal port caused by the tangential configuration of the radiation beams. This underdosed area also extended beyond the ROI, which can be clinically relevant as the tissue directly abutting the implant is a probable location of local recurrence. ${ }^{59}$

For the ROI (defined as a $5 \mathrm{~mm}$ expansion around the saline implant), the ranges in percent point dose changes were $+8 \%$ to $-5 \%$ for 3 DCRT (MC) and, $+10 \%$ to $-6 \%$ for 3DCRT (CC). Smaller variation in point dose differences in the ROI were observed in the VMAT (MC) and helical Tomotherapy (CS) plans with changes varying from $-2 \%$ to $3.5 \%$ and $-1.5 \%$ to $5 \%$, respectively.

For a comparison of a clinically relevant metric, the DVH of the ROI is shown in Figure 5.4, comparing the original DVH curve to the cumulative DVH curve when daily measured internal port errors are modelled in all three techniques. From Figure 5.4, the change in $\mathrm{V}_{100 \% \mathrm{Rx}}$ (volume receiving $100 \%$ of $\mathrm{Rx}$ ) of the ROI was calculated for each of the three techniques, and was found to be smaller than $-1.7 \%$ in all three techniques. Similar DVH curves were generated for the ipsilateral lung and the heart which showed no discernible changes in the OARs. Therefore, large internal port errors had minimal effect on target coverage and no effect on OARs.

The underdosage that is seen in the percent dose different maps for the 3DCRT plan (Figure 5.3) is not explicitly visible in the DVH of Figure 5.4. This is because the original tangential 3DCRT plan contains a light, lower-dose streak in the shadow of the port (near the chest-wall). As a result, the DVH curve of the original tangential 3DCRT plan was inferior to the VMAT and helical Tomotherapy (Figure 5.4). When daily variable internal port errors were modelled, the metal port was flipped to the anterior end of the breast for more than half of the fractions. This has resulted in an underdosed streak near the skin, 
while compensating for the original underdosed streak near the chest-wall. Therefore, in both scenarios the number of voxels that were underdosed was similar (while the underdosage streaks were present in different areas), and the resultant DVH curves had a similar shape.
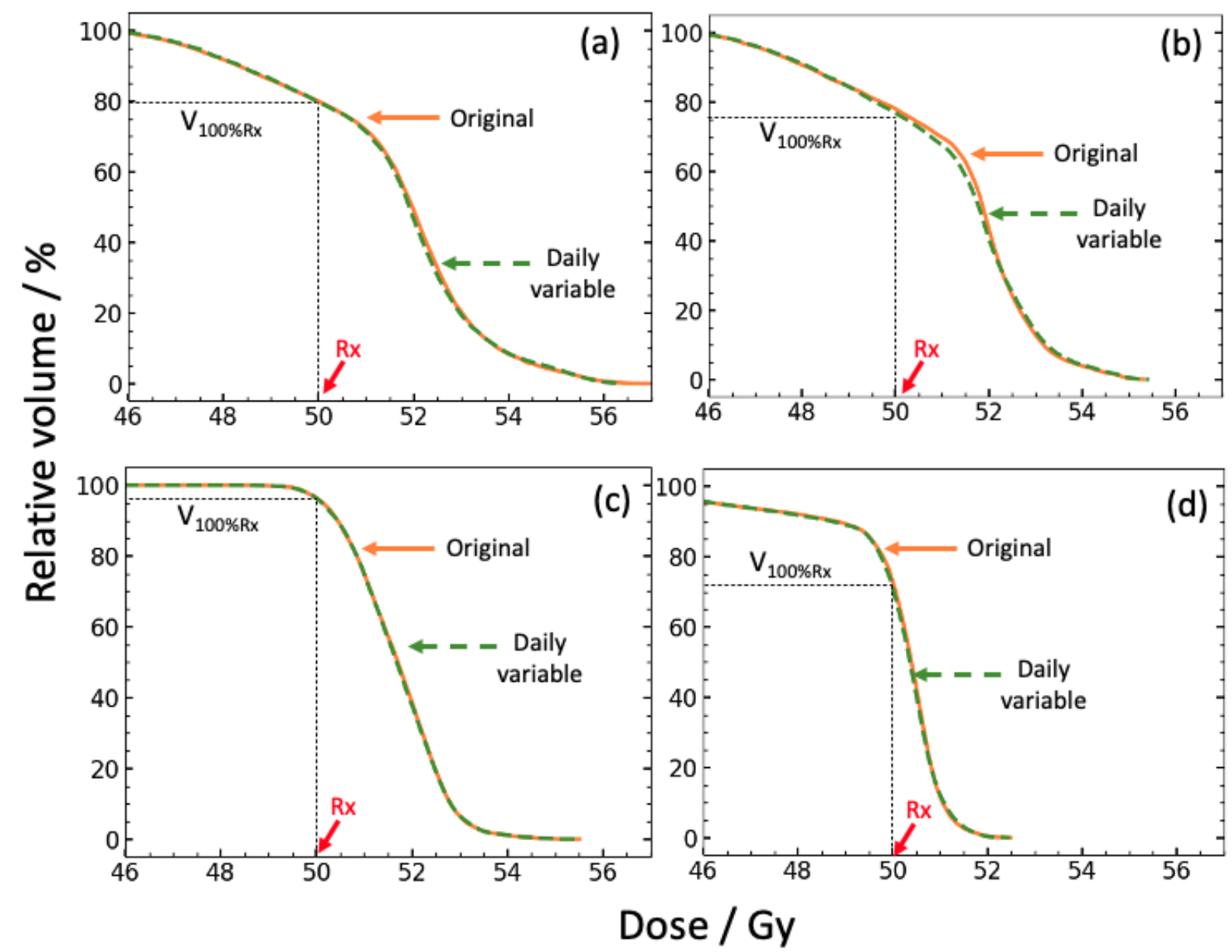

Figure 5.4: DVH of the ROI for the special patient when internal port errors were modelled in 3DCRT MC (a), 3DCRT CC (b), VMAT MC (c) and helical Tomotherapy CS (d). Solid orange: original plan. Green dashed: cumulative dose with daily variable port error modelled. 
The recent recall of one of the main textured implants available in the market due to the increased risk of breast implant-associated anaplastic large cell lymphoma (BIAALCL) may potentially increase the use of smooth tissue expanders. ${ }^{63,64}$ As migration is more of a concern with smooth tissue expanders, this special case shows that large internal positional variations of the metal port have no clinically relevant effect on target coverage and OARs for VMAT and helical Tomotherapy treatments. However, a treatment with tangential 3DCRT may produce an underdosage in the shadow of the metal port as observed in the underdosage streak in Figure 5.3. Depending on the initial location of the metal port in the simulation scan and the locations it migrates to, the skin and tissue abutting the implant can be underdosed and potentially undermine the treatment efficacy.

\subsection{Summary}

The magnitude of the inter-fractional positional variations of the metal port was measured for a special patient. For this special case, the tissue expander was loose within the breast pocket, which led to internal port shifts larger than $6 \mathrm{~cm}$. The cumulative dosimetric effect of the daily variable internal port error was quantified in different techniques. In VMAT and helical Tomotherapy treatments, the large internal port errors affected the dose distribution within the non-biological tissue expander and had minimal effects on target coverage and OARs. On the other hand, in the tangential 3DCRT treatment, the large internal port errors resulted in underdosed areas in the skin. These results indicate that smooth implants can potentially replace the traditional textured implants for postmastectomy reconstruction for patients treated with VMAT or helical Tomotherapy. 


\section{Chapter 6: Overall summary, conclusions and future work}

Summary. Some postmastectomy patients that undergo an immediate breast reconstruction receive a radiation treatment when the temporary tissue expander is in place. The tissue expander contains a high-density metal port that is localized with an external magnet and used as an injection port for saline solution. Perturbations in the dose distribution occur if the presence of the high-density metal port is not accurately modelled in the treatment planning system calculations, or if its location at the time of treatment is different from its location at the time of the simulation scan that is used for treatment planning. In this study, the magnitude and the dosimetric impact of the inter-fractional positional variations of the metal port was quantified for a cohort of patients. The robustness of the three mostcommon external beam treatment techniques (tangential 3DCRT, VMAT and helical Tomotherapy) against the measured inter-fractional errors were compared. Relative changes in target coverage and doses to relevant OARs were analyzed and compared for each technique when daily variable and systematic port positional variations were simulated.

Results showed that port positional errors are generally small and centered around zero, but errors larger than $1.5 \mathrm{~cm}$ can also be observed. The measured positional errors were classified as internal port errors (migration of the port relative to internal anatomy), or patient registration error (shift of the whole patient relative to the treatment beam).

Internal port errors were shown to have small effects on the target, and no discernable effect was observed on the OARs. On the other hand, patient registration errors were shown to have a larger dosimetric effect on the target coverage and OARs in all three techniques. When systematic internal port errors were modelled, VMAT and helical 
Tomotherapy plans were more robust against these errors compared with the tangential configuration of a 3DCRT plan. On the other hand, tangential 3DCRT plans were more robust against patient registration errors with regard to target coverage and dose to OARs.

Overriding the metal port with tissue density as a potential planning strategy was found to overestimate the dose to the ROI in all three treatment techniques. These results suggest that ignoring the density of the metal port during treatment planning can lead to a clinically significant underdosage of the ROI.

For the special patient that was investigated separately, the position of the metal port varied substantially, with a maximum displacement of $6.3 \mathrm{~cm}$ in one direction. The cumulative dosimetric effect of the large internal port errors resulted in small changes to target coverage and OARs for VMAT and helical Tomotherapy plans. For the tangential 3DCRT plan, the large displacements of the metal port resulted in an area of underdosage near the skin.

Conclusions. In reality, the inter-fractional positional variations of the metal port arise from a combination of internal port errors and registration errors. The results of this study indicate that VMAT and helical Tomotherapy treatments are more robust when patient registration errors are minimized. The cumulative dosimetric effect of daily variable and systematic internal port errors on target coverage and OARs was found to be very small in the three treatment techniques. Therefore, in treatment delivery systems where daily image guidance is used and the metal port is visible, therapists should not compromise the accuracy of matching anatomical landmarks for a better alignment of the metal port, regardless of the treatment technique. In addition, contouring the metal port with some 
uncertainty and assigning its components the appropriate densities better represents the real course of treatment in all three techniques, as opposed to overriding the metal port with tissue-equivalent density. Lastly, the use of smooth tissue expanders instead of textured implants is a reasonable approach dosimetrically in VMAT and helical Tomotherapy, as the potential migration of the metal port throughout the course of the treatment was shown to have minimal dosimetric effects in these techniques.

Future work. In this study, the most commonly used tissue expander was investigated, however, other models exist or are emerging in the market. For example, the AeroForm tissue expander (AirXpanders, Palo Alto, CA, USA) contains a stainless-steel reservoir of compressed $\mathrm{CO} 2$. The implant is expanded by gradual release of $\mathrm{CO} 2$ to the silicon implant, which is remotely controlled by the patient. The inter-fractional positional variation and the dosimetric impact of the reservoir needs to be examined, as its shape and position within the implant is different than the metal port presented in this study.

Each dose calculation engine in a TPS is commissioned before clinical use. If that engine is to be used for calculations around tissue interfaces, then interface dosimetry is part of the commissioning process. In addition, it is crucial to evaluate the accuracy of the engine for specific situations of interface dosimetry, such as the presence of the highdensity metal port. This exercise has been reported previously in the literature for different TPSs and different treatment configurations. A common approach is to compare TPS calculations to experimental measurements and adjust the density of the metal port within the TPS until the calculation result matches the experimental measurements. This adjustment is done to compensate for the limitations of commercial TPSs around the tissue 
inhomogeneity. A clinical exercise of this nature is currently underway at TOHCC, led by the author of this thesis. 


\section{Appendix}

In this appendix, a list of the anonymized patients included in this study is presented. The outer blue contour is the PTV, the red contour is the core magnet and the green contour is the titanium shell. The last patient indexed as 9 is the special case patient that was investigated separately in Chapter 5 . The window/level is adjusted to minimize the appearance of the metal artefacts. R: Right breast, L: Left breast.

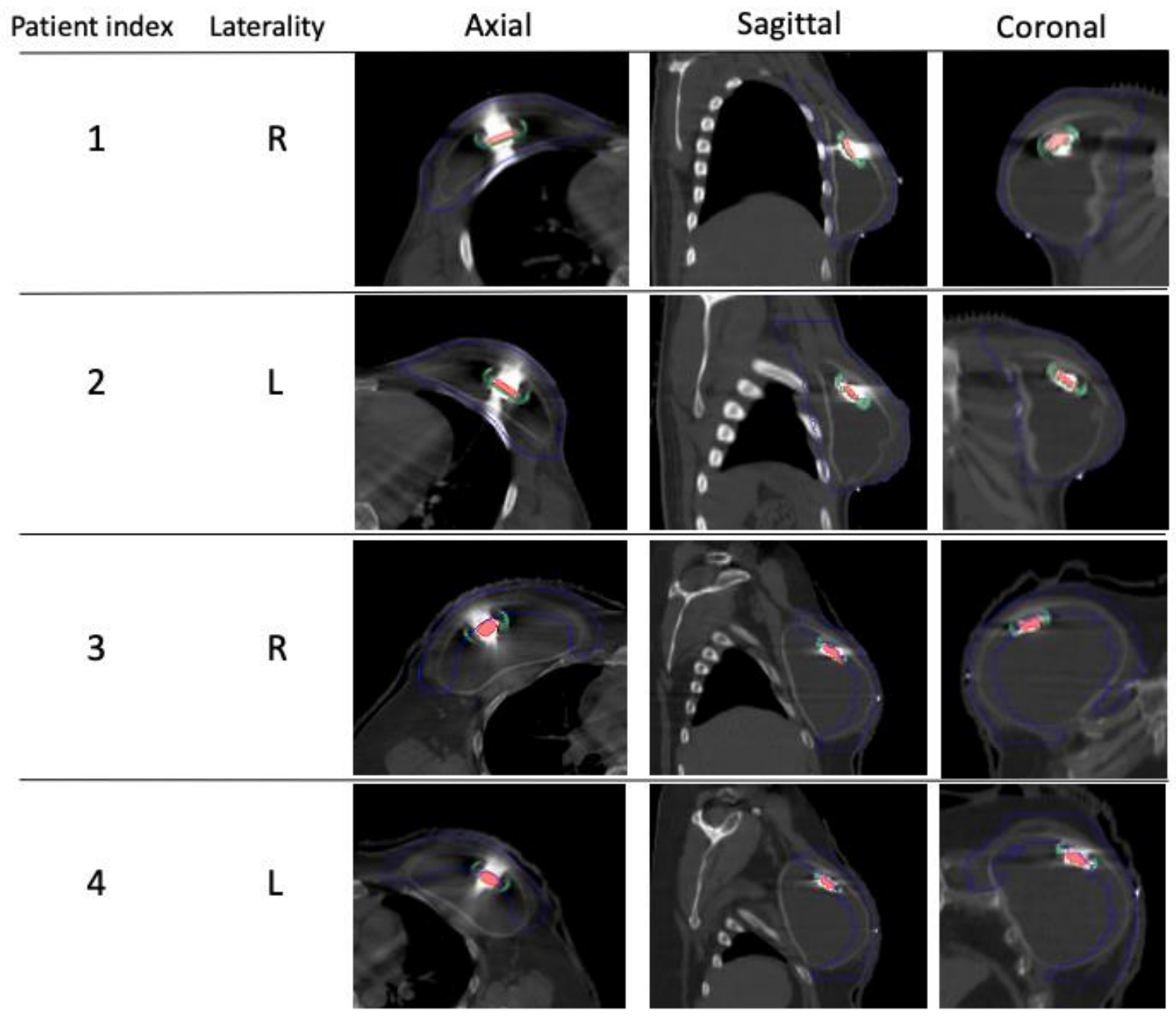




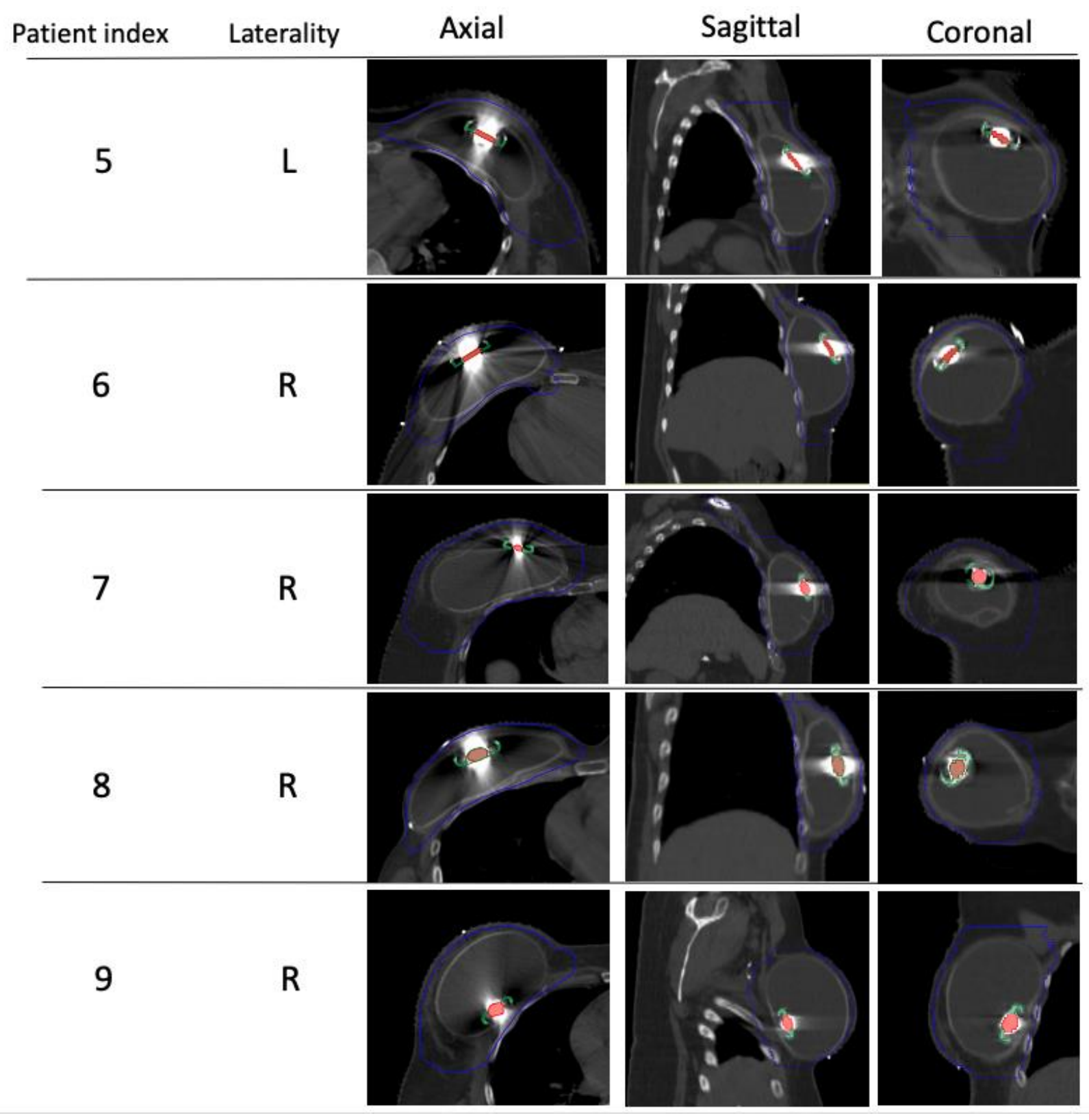




\section{References}

[ 1 ] D.R. Brenner, H.K. Weir, A.A. Demers, L.F. Ellison, C. Louzado, A. Shaw, D. Turner, R.R. Woods, and L.M. Smith, Projected estimates of cancer in Canada in 2020, CMAJ 192(9), E199-E205 (2020).

[2 $]$ C.C.S.A. Committee, Canadian cancer statistics 2019. Toronto: Canadian Cancer Society; 2019, (n.d.).

[ 3 ] G.N. Sharma, R. Dave, J. Sanadya, P. Sharma, and K.K. Sharma, Various types and management of breast cancer: An overview, J. Adv. Pharm. Technol. Res. 1(2), 109$126(2010)$.

[ 4 ] U. Veronesi, N. Cascinelli, L. Mariani, M. Greco, R. Saccozzi, A. Luini, M. Aguilar, and E. Marubini, Twenty-Year Follow-up of a Randomized Study Comparing Breast-Conserving Surgery with Radical Mastectomy for Early Breast Cancer, N. Engl. J. Med. 347(16), 1227-1232 (2002).

[ 5 ] C.R. Albornoz, E. Matros, C.N. Lee, C.A. Hudis, A.L. Pusic, E. Elkin, P.B. Bach, P.G. Cordeiro, and M. Morrow, Bilateral mastectomy versus breast-conserving surgery for early-stage breast cancer: the role of breast reconstruction, Plast. Reconstr. Surg. 135(6), 1518-1526 (2015).

[ 6 ] H. Panchal and E. Matros, Current trends in postmastectomy breast reconstruction, Plast. Reconstr. Surg. 140(5S), 7S-13S (2017).

[ 7 ] N. Ilonzo, A. Tsang, S. Tsantes, A. Estabrook, and A.M. Thu Ma, Breast reconstruction after mastectomy: A ten-year analysis of trends and immediate postoperative outcomes, Breast 32, 7-12 (2017).

[ 8 ] C.R. Albornoz, P.B. Bach, B.J. Mehrara, J.J. Disa, A.L. Pusic, C.M. McCarthy, P.G. Cordeiro, and E. Matros, A paradigm shift in U.S. Breast reconstruction: Increasing implant rates, Plast. Reconstr. Surg. 131(1), 15-23 (2013).

[ 9 ] C. Radovan, Breast reconstruction after mastectomy using the temporary expander, Plast. Reconstr. Surg. 69(2), 195-208 (1982).

[ 10 ] C.W. Taylor, K. Horgan, and D. Dodwell, Oncological aspects of breast reconstruction, The Breast 14(2), 118-130 (2005).

[11 ] P.M. Chevray, Timing of breast reconstruction: immediate versus delayed, Cancer J. 14(4), 223-229 (2008).

[ 12 ] E.E. Elder, Y. Brandberg, T. Björklund, R. Rylander, J. Lagergren, G. Jurell, M. Wickman, and K. Sandelin, Quality of life and patient satisfaction in breast cancer patients after immediate breast reconstruction: A prospective study, Breast 14(3), 201-208 (2005).

[13 ] R. Baskar, K.A. Lee, R. Yeo, and K.W. Yeoh, Cancer and radiation therapy: Current advances and future directions, Int. J. Med. Sci. 9(3), 193-199 (2012).

[ 14 ] F.M. Khan and J.P. Gibbons, The physics of radiadtion therapy, 5th ed. (Lippincott Williams \& Wilkins, 2014).

[ 15 ] G.C. Pereira, M. Traughber, and R.F. Muzic, The role of imaging in radiation therapy planning: Past, present, and future, Biomed Res. Int. 2014, 231090 (2014).

[16] P. Suetens (ed.), X-ray computed tomography, in Fundam. Med. Imaging, 2nd ed.(Cambridge University Press, Cambridge, 2009), pp. 33-63.

[17 ] F.M. Khan, J.P. Gibbons, and P.W. Sperduto, Khan's Treatment Planning in Radiation Oncology (Lippincott Williams \& Wilkins, 2016). 
[ 18 ] J. Sykes, Reflections on the current status of commercial automated segmentation systems in clinical practice, J. Med. Radiat. Sci. 61(3), 131-134 (2014).

[ 19 ] G. Sharp, K.D. Fritscher, V. Pekar, M. Peroni, N. Shusharina, H. Veeraraghavan, and J. Yang, Vision 20/20: perspectives on automated image segmentation for radiotherapy, Med. Phys. 41(5), 50902 (2014).

[20 ] Report 83: Prescribing, recording, and reporting photon-beam intensity-modulated radiation therapy (IMRT), J. ICRU 10(1), 1-106 (2010).

[ 21 ] J.A. Purdy, Radiotherapy Accessories, in Encycl. Med. Devices Instrum.(John Wiley \& Sons, Inc., Hoboken, NJ, USA, 2006).

[ 22 ] R.E. Drzymala, R. Mohan, L. Brewster, J. Chu, M. Goitein, W. Harms, and M. Urie, Dose-volume histograms, Int. J. Radiat. Oncol. 21(1), 71-78 (1991).

[ 23 ] P.R. Symonds, J.A. Mills, and A. Duxbury, Walter and Miller's Textbook of Radiotherapy: Radiation Physics, Therapy and Oncology-E-Book (Elsevier Health Sciences, 2019).

[ 24 ] A. Boyer, P. Biggs, J. Galvin, E. Klein, T. LoSasso, D. Low, K. Mah, and C. Yu, Basic applications of multileaf collimators. AAPM Radiation Therapy Committee Task Group No. 50 Report No. 72, (2001).

[25] Y. Nishimura and R. Komaki, Intensity-modulated radiation therapy: clinical evidence and techniques (Springer, 2015).

[ 26 ] K. Otto, Volumetric modulated arc therapy: IMRT in a single gantry arc, Med. Phys. 35(1), 310-317 (2008).

[ 27 ] C.C. Popescu, I.A. Olivotto, W.A. Beckham, W. Ansbacher, S. Zavgorodni, R. Shaffer, E.S. Wai, and K. Otto, Volumetric modulated arc therapy improves dosimetry and reduces treatment time compared to conventional intensitymodulated radiotherapy for locoregional radiotherapy of left-sided breast cancer and internal mammary nodes, Int. J. Radiat. Oncol. 76(1), 287-295 (2010).

[28 ] P.M. Haertl, F. Pohl, K. Weidner, C. Groeger, O. Koelbl, and B. Dobler, Treatment of left sided breast cancer for a patient with funnel chest: Volumetric-modulated arc therapy vs. 3D-CRT and intensity-modulated radiotherapy, Med. Dosim. 38(1), 14 (2013).

[ 29 ] G.-H. Jin, L.-X. Chen, X.-W. Deng, X.-W. Liu, Y. Huang, and X.-B. Huang, A comparative dosimetric study for treating left-sided breast cancer for small breast size using five different radiotherapy techniques: conventional tangential field, filed-in-filed, Tangential-IMRT, Multi-beam IMRT and VMAT, Radiat. Oncol. 8(1), 89 (2013).

[ 30 ] T.R. Mackie, T. Holmes, S. Swerdloff, P. Reckwerdt, J.O. Deasy, J. Yang, B. Paliwal, and T. Kinsella, Tomotherapy: A new concept for the delivery of dynamic conformal radiotherapy, Med. Phys. 20(6), 1709-1719 (1993).

[ 31 ] V.J. Gonzalez, D.J. Buchholz, K.M. Langen, G.H. Olivera, B. Chauhan, S.L. Meeks, K.J. Ruchala, J. Haimerl, W. Lu, and P.A. Kupelian, Evaluation of two tomotherapybased techniques for the delivery of whole-breast intensity-modulated radiation therapy, Int. J. Radiat. Oncol. Biol. Phys. 65(1), 284-290 (2006).

[ 32 ] G.X. Zhou, S.P. Xu, X.K. Dai, Z.J. Ju, H.S. Gong, C.B. Xie, L.M. Yin, and J. Yang, Clinical dosimetric study of three radiotherapy techniques for postoperative breast cancer: Helical Tomotherapy, IMRT, and 3D-CRT, Technol. Cancer Res. Treat. 10(1), 15-23 (2011). 
[ 33 ] S.M. Goddu, S. Chaudhari, M. Mamalui-Hunter, O.L. Pechenaya, D. Pratt, S. Mutic, I. Zoberi, S. Jeswani, S.N. Powell, and D.A. Low, Helical Tomotherapy planning for left-sided breast cancer patients with positive lymph nodes: comparison to conventional multiport breast technique, Int. J. Radiat. Oncol. 73(4), 1243-1251 (2009).

[ 34 ] J.-M. Caudrelier, S.C. Morgan, L. Montgomery, M. Lacelle, B. Nyiri, and M. MacPherson, Helical tomotherapy for locoregional irradiation including the internal mammary chain in left-sided breast cancer: Dosimetric evaluation, Radiother. Oncol. 90(1), 99-105 (2009).

[ 35 ] C. Ma and L. Wang, Point Dose Kernel, in Encycl. Radiat. Oncol., edited by L.W. Brady and T.E. Yaeger (Springer Berlin Heidelberg, Berlin, Heidelberg, 2013), p. 636.

[36] A. Ahnesjö, Collapsed cone convolution of radiant energy for photon dose calculation in heterogeneous media, Med. Phys. 16(4), 577-592 (1989).

[ 37 ] D.W.O. Rogers and A.F. Bielajew, Monte Carlo techniques of electron and photon transport for radiation dosimetry, Dosim. Ioniz. Radiat. 3, 427-539 (1990).

[ 38 ] M. Overgaard, P.S. Hansen, J. Overgaard, C. Rose, M. Andersson, F. Bach, M. Kjaer, C.C. Gadeberg, H.T. Mouridsen, M.-B. Jensen, and K. Zedeler, Postoperative radiotherapy in high-risk premenopausal women with breast cancer who receive adjuvant chemotherapy, N. Engl. J. Med. 337(14), 949-955 (1997).

[ 39 ] P. McGale, C. Taylor, C. Correa, D. Cutter, F. Duane, M. Ewertz, R. Gray, G. Mannu, R. Peto, and T. Whelan, Effect of radiotherapy after mastectomy and axillary surgery on 10-year recurrence and 20-year breast cancer mortality, Lancet 383(9935), 2127-2135 (2014).

[ 40 ] J. Ragaz, I.A. Olivotto, J.J. Spinelli, N. Phillips, S.M. Jackson, K.S. Wilson, M.A. Knowling, C.M.L. Coppin, L. Weir, K. Gelmon, N. Le, R. Durand, A.J. Coldman, and M. Manji, Locoregional radiation therapy in patients with high-risk breast cancer receiving adjuvant chemotherapy: 20-year results of the British Columbia randomized trial, JNCI J. Natl. Cancer Inst. 97(2), 116-126 (2005).

[ 41 ] J. Moni, M. Graves-Ditman, P. Cederna, K. Griffith, E.A. Krueger, B.A. Fraass, and L.J. Pierce, Dosimetry around metallic ports in tissue expanders in patients receiving postmastectomy radiation therapy: An ex vivo evaluation, Med. Dosim. 29(1), 4954 (2004).

[ 42 ] R.C.A. Thompson and A.M. Morgan, Investigation into dosimetric effect of a MAGNA-SITE ${ }^{\mathrm{TM}}$ tissue expander on post-mastectomy radiotherapy, Med. Phys. 32(6Part1), 1640-1646 (2005).

[ 43 ] S. Damast, K. Beal, Å. Ballangrud, T.J. Losasso, P.G. Cordeiro, J.J. Disa, L. Hong, and B.L. McCormick, Do metallic ports in tissue expanders affect postmastectomy radiation delivery?, Int. J. Radiat. Oncol. Biol. Phys. 66(1), 305-310 (2006).

[ 44 ] C. Chatzigiannis, G. Lymperopoulou, P. Sandilos, C. Dardoufas, E. Yakoumakis, E. Georgiou, and P. Karaiskos, Dose perturbation in the radiotherapy of breast cancer patients implanted with the Magna-Site: a Monte Carlo study, J. Appl. Clin. Med. Phys. 12(2), 58-70 (2011).

[ 45 ] S.A. Chen, T. Ogunleye, A. Dhabbaan, E.H. Huang, A. Losken, S. Gabram, L. Davis, and M.A. Torres, Impact of internal metallic ports in temporary tissue expanders on postmastectomy radiation dose distribution, Int. J. Radiat. Oncol. Biol. 
Phys. 85(3), 630-635 (2013).

[ 46 ] L. Koutcher, Å. Ballangrud, P.G. Cordeiro, B. McCormick, M. Hunt, K.J.V. Zee, C. Hudis, and K. Beal, Postmastectomy intensity modulated radiation therapy following immediate expander-implant reconstruction, Radiother. Oncol. 94(3), 319-323 (2010).

[ 47 ] D.M. Trombetta, S.C. Cardoso, A. Facure, A.X. da Silva, and L.A.R. da Rosa, Influence of the presence of tissue expanders on energy deposition for postmastectomy radiotherapy, PLoS One 8(2), (2013).

[ 48 ] B. Strang, K. Murphy, S. Seal, and A. Dal Cin, Does the presence of an implant including expander with internal port alter radiation dose? An ex vivo model, Can. J. Plast. Surg. 21(1), 37-40 (2013).

[49] A. Asena, T. Kairn, S.B. Crowe, and J. V. Trapp, Establishing the impact of temporary tissue expanders on electron and photon beam dose distributions, Phys. Medica 31(3), 281-285 (2015).

[ 50 ] D.M. Trombetta, S.C. Cardoso, V.G.L. Alves, A. Facure, D.V.S. Batista, and A.X. Da Silva, Evaluation of the radiotherapy treatment planning in the presence of a magnetic valve tissue expander, PLoS One 10(2), e0117548 (2015).

[ 51 ] H.E. Gee, F. Bignell, D. Odgers, S. Gill, D. Martin, J. Toohey, and S. Carroll, In vivo dosimetric impact of breast tissue expanders on post-mastectomy radiotherapy, J. Med. Imaging Radiat. Oncol. 60(1), 138-145 (2016).

[ 52 ] J.M. Park, K. Kim, J.I. Park, K.H. Shin, U.S. Jin, and J.I. Kim, Dosimetric effect of internal metallic ports in temporary tissue expanders on postmastectomy radiation therapy: A Monte Carlo study, Phys. Med. Biol. 62(12), 4623-4636 (2017).

[ 53 ] M.F. da Silva, H.F. de Oliveira, L.F. Borges, H.H.A. Carrara, and J.A. Farina, Effects of the metallic port in tissue expanders on dose distribution in postmastectomy radiotherapy, Ann. Plast. Surg. 80(1), 67-70 (2018).

[ 54 ] J. Yoon, Y. Xie, D. Heins, and R. Zhang, Modeling of the metallic port in breast tissue expanders for photon radiotherapy, J. Appl. Clin. Med. Phys. 19(3), 205-214 (2018).

[ 55 ] N. Mizuno, H. Takahashi, J. Kawamori, N. Nakamura, M. Ogita, S. Hatanaka, R. Yamauchi, M. Hariu, and K. Sekiguchi, Determination of the appropriate physical density of internal metallic ports in temporary tissue expanders for the treatment planning of post-mastectomy radiation therapy, J. Radiat. Res. 59(2), 190-197 (2018).

[ 56 ] F.E. Boas and D. Fleischmann, CT artifacts: Causes and reduction techniques, J. Med. Imaging 4(2), 229-240 (2012).

[ 57 ] R.P. Smith, P. Bloch, E.E. Harris, J. McDonough, A. Sarkar, A. Kassaee, S. Avery, and L.J. Solin, Analysis of interfraction and intrafraction variation during tangential breast irradiation with an electronic portal imaging device, Int. J. Radiat. Oncol. Biol. Phys. 62(2), 373-378 (2005).

[ 58 ] L. Genton, U.G. Kyle, S. Balmer Majno, and C. Pichard, Body composition changes in breast cancer patients during curative radiation therapy, e-SPEN 1(1), 2-8 (2006).

[ 59 ] H.N. Langstein, M.-H. Cheng, S.E. Singletary, G.L. Robb, E. Hoy, T.L. Smith, and S.S. Kroll, Breast cancer recurrence after immediate reconstruction: patterns and significance, Plast. Reconstr. Surg. 111(2), 712-722 (2003).

[60] P. Mildenberger, M. Eichelberg, and E. Martin, Introduction to the DICOM 
standard, Eur. Radiol. 12(4), 920-927 (2002).

[ 61 ] D. Mason, SU-E-T-33: Pydicom: An Open Source DICOM Library, Med. Phys. 38(6Part10), 3493 (2011).

[ 62 ] G.P. Maxwell, M. Scheflan, S. Spear, M.B. Nava, and P. Hedén, Benefits and limitations of macrotextured breast implants and consensus recommendations for optimizing their effectiveness, Aesthetic Surg. J. 34(6), 876-881 (2014).

[63 ] R. Tevlin, M.R. Borrelli, A.D. Azad, V. Posternak, D.C. Wan, D.H. Nguyen, and R. Nazerali, Examination of smooth versus textured Ttssue expanders in breast reconstruction, Plast. Reconstr. Surg. - Glob. Open 7, 37-38 (2019).

[64] Allergan Recalls Natrelle Biocell Textured Breast Implants Due to Risk of BIAALCL Cancer IFDA, (2019). 